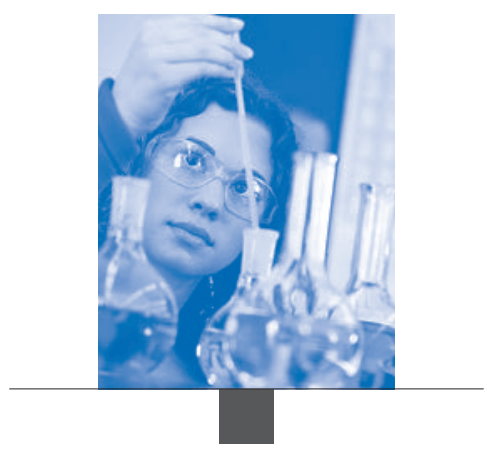

\title{
Annexe A
}

\section{QUESTIONNAIRES CONTEXTUELS DE L'ENQUÊTE PISA 2015}

L'annexe A présente les questionnaires contextuels administrés dans le cadre de l'enquête PISA 2015, à savoir : le questionnaire « Établissement ", administré aux chefs d'établissement ; le questionnaire " Élève ", administré à tous les élèves participants ; deux questionnaires facultatifs à l'intention des élèves - le questionnaire sur le parcours scolaire et le questionnaire sur la maîtrise des technologies de l'information et de la communication ; et enfin, deux questionnaires facultatifs - le questionnaire « Parents » et le questionnaire " Enseignants ». 


\section{QUESTIONNAIRE ÉTABLISSEMIENT}

\section{Version en ligne/version source pour la campagne définitive}

Un questionnaire "Établissement » est administré aux chefs d'établissement. Environ 60 minutes sont nécessaires pour le remplir. Il est destiné à recueillir des renseignements sur :

- L'établissement en général

- La gestion de l'établissement

- Le personnel enseignant

- L'évaluation

- Des groupes cibles

- Le climat de l'établissement

Les termes techniques sont indiqués entre <crochets> et adaptés au contexte national par le centre national de collecte de données des pays ou économies participants. Dans cette annexe, ces termes techniques sont expliqués sous les items.

\section{Informations générales sur l'établissement}

SC001

SC001Q01TA
Comment peut-on le mieux définir la collectivité dans laquelle se situe votre établissement ?

(Sélectionnez une réponse.)

\begin{tabular}{|l|c}
\hline Un village, hameau oucollectivité rurale (moins de 3 000 habitants) & $\square_{1}$ \\
\hline Un bourg (de 3000 à 15000 habitants environ) & $\square_{2}$ \\
\hline Une petite ville (de 15000 à 100 000 habitants environ) & $\square_{3}$ \\
\hline Une ville (entre 100000 et un million d'habitants environ) & $\square_{4}$ \\
\hline Une grande agglomération de plus d'un million d'habitants & $\square$ \\
\hline
\end{tabular}

\section{SC002}

SC002Q01TA

SC002Q02TA

SC003

SC003Q01TA
Le $<1^{\text {er }}$ février 2015>, quel était le nombre total d'élèves inscrits dans votre établissement ?

(Tapez un nombre sur chaque ligne. Tapez " 0 » (zéro) s'il n’y en a aucun(e). Nombre de garçons :

Nombre de filles:

Quelle est, dans votre établissement, la taille moyenne d'une classe de <langue de l'évaluation> de < grade modal du pays pour les élèves de 15 ans $>$ ?

(Sélectionnez une réponse.)

\begin{tabular}{|l|c}
\hline 15 élèves ou moins & $\square_{1}$ \\
\hline De 16 à 20 élèves & $\square_{2}$ \\
\hline De 21 à 25 élèves & $\square_{3}$ \\
\hline De 26 à 30 élèves & $\square_{4}$ \\
\hline De 31 à 35 élèves & $\square_{5}$ \\
\hline De 36 à 40 élèves & $\square_{6}$ \\
\hline De 41 à 45 élèves & $\square_{7}$ \\
\hline De 46 à 50 élèves & $\square_{8}$ \\
\hline Plus de 50 élèves & $\square_{9}$ \\
\hline
\end{tabular}


L'objectif des questions suivantes est d'estimer le nombre d'élèves de <grade modal du pays pour les élèves de 15 ans> par ordinateur dans votre établissement.

(Tapez un nombre par ligne. Tapez « 0 » (zéro) s'il n'y en a aucun.)

\begin{tabular}{c|l|l}
\hline \multicolumn{2}{l}{} & \multicolumn{2}{l}{ Nombre } \\
\hline SC004Q01TA & $\begin{array}{l}\text { Dans votre établissement, quel est le nombre total d'élèves de <grade modal du pays pour les élèves } \\
\text { de } 15 \text { ans }>\text { ? }\end{array}$ & $\begin{array}{l}\text { Quel est le nombre approximatif d'ordinateurs qui sont mis à la disposition de ces élèves à des fins } \\
\text { d'enseignement? }\end{array}$ \\
\hline SC004Q02TA & Quel est le nombre approximatif de ces ordinateurs qui sont connectés à Internet / au réseau web mondial ? & \\
\hline SC004Q04NA & $\begin{array}{l}\text { Quel est le nombre approximatif de ces ordinateurs qui sont de type portable (par ex. ordinateur portable, } \\
\text { tablette) ? }\end{array}$ & \\
\hline SC004Q05NA & Environ combien de tableaux blancs interactifs sont-ils disponibles au total dans l'établissement? \\
\hline SC004Q06NA & Environ combien de vidéoprojecteurs sont-ils disponibles au total dans l'établissement? & $\begin{array}{l}\text { Environ combien d'ordinateurs équipés d'une connexion Internet sont-ils réservés aux enseignants dans votre } \\
\text { SC004Q07ablissement? }\end{array}$ \\
\hline
\end{tabular}

Parmi les activités suivantes, lesquelles votre établissement propose-t-il aux élèves de <grade modal du pays pour les élèves de 15 ans> <cette année scolaire-ci> ? (Sélectionnez une réponse par ligne.)

\begin{tabular}{|c|c|c|c|}
\hline & & Oui & Non \\
\hline SC053Q01TA & Fanfare, orchestre ou chorale & $\square_{1}$ & $\square_{2}$ \\
\hline SC053Q02TA & Pièce de théâtre ou comédie musicale & $\square_{1}$ & $\square_{2}$ \\
\hline SC053Q03TA & Rédaction de l'album-souvenir annuel, du journal ou de la revue de l'établissement & $\square_{1}$ & $\square_{2}$ \\
\hline SC053Q04TA & Bénévolat ou activités humanitaires, par ex. <exemples nationaux> & $\square_{1}$ & $\square_{2}$ \\
\hline SC053Q05NA & Club de sciences & $\square_{1}$ & $\square_{2}$ \\
\hline SC053Q06NA & Compétitions de sciences, par ex. <exemples nationaux> & $\square_{1}$ & $\square_{2}$ \\
\hline SC053Q07TA & Club d'échecs & $\square_{1}$ & $\square_{2}$ \\
\hline SC053Q08TA & Club axé sur l'informatique/les technologies de l'information et de la communication (TIC) & $\square_{1}$ & $\square_{2}$ \\
\hline SC053Q09TA & Club artistique ou activités artistiques & $\square_{1}$ & $\square_{2}$ \\
\hline SC053Q10TA & Équipe sportive ou activités sportives & $\square_{1}$ & $\square_{2}$ \\
\hline SC053Q11TA & $<$ ltem national $>$ & $\square_{1}$ & $\square_{2}$ \\
\hline
\end{tabular}

Concernant la section des sciences dans votre établissement, les affirmations suivantes sont-elles vraies?

(Sélectionnez une réponse par ligne.)

\begin{tabular}{l|l|c|c}
\multicolumn{1}{l|}{} & Oui & Non \\
\hline SC059Q01NA & $\begin{array}{l}\text { Par rapport aux autres sections, la <section des cours de sciences> de l'établissement est } \\
\text { bien équipée. }\end{array}$ & $\square_{1}$ & $\square_{2}$ \\
\hline SC059Q02NA & $\begin{array}{l}\text { Lorsque nous disposons de fonds supplémentaires, une grande partie est consacrée } \\
\text { à améliorer l'enseignement des <cours de sciences>. }\end{array}$ & $\square_{1}$ & $\square_{2}$ \\
\hline SC059Q03NA & Les enseignants de <cours de sciences> figurent parmi nos enseignants les plus qualifiés. & $\square_{1}$ & $\square_{2}$ \\
\hline SC059Q04NA & $\begin{array}{l}\text { Par rapport à des établissements comparables, nous disposons d'un laboratoire bien } \\
\text { équipé. }\end{array}$ & $\square_{1}$ & $\square_{2}$ \\
\hline SC059Q05NA & Le matériel destiné aux travaux pratiques de <cours de sciences> est en bon état. & $\square_{1}$ & $\square_{2}$ \\
\hline SC059Q06NA & $\begin{array}{l}\text { Nous disposons de suffisamment de matériel de laboratoire pour permettre son utilisation } \\
\text { régulière à tous les cours. }\end{array}$ & $\square_{1}$ & $\square_{2}$ \\
\hline SC059Q07NA & $\begin{array}{l}\text { Nous disposons de personnel auxiliaire de laboratoire pour nous aider } \\
\text { dans l'enseignement des <cours de sciences>. }\end{array}$ & $\square_{1}$ \\
\hline SC059Q08NA & $\begin{array}{l}\text { Notre établissement engage des dépenses supplémentaires pour l'achat de matériel récent } \\
\text { pour le <cours de sciences>. }\end{array}$ & $\square_{1}$ \\
\hline
\end{tabular}


SC052

Votre établissement propose-t-il les dispositifs suivants d'aide à l'étude aux élèves de $\mathbf{1 5}$ ans ? (Sélectionnez une réponse par ligne.)

\begin{tabular}{l|l|c|c}
\multicolumn{2}{l|}{} & Oui & Non \\
\hline SC052Q01NA & Une ou plusieurs salles où les élèves peuvent faire leurs devoirs & $\square_{1}$ & $\square_{2}$ \\
\hline SC052Q02NA & Une aide aux devoirs faite par le personnel & $\square_{1}$ & $\square_{2}$ \\
\hline
\end{tabular}

\section{Gestion de l'établissement}

\begin{tabular}{|c|c|c|c|c|c|c|c|}
\hline \multirow[t]{2}{*}{ SC009 } & \multicolumn{7}{|c|}{$\begin{array}{l}\text { Vous trouverez ci-dessous des affirmations concernant votre gestion de l'établissement. } \\
\text { Veuillez indiquer la fréquence à laquelle vous avez adopté les attitudes suivantes } \\
\text { dans l'établissement lors de <l'année scolaire en cours / I'année scolaire écoulée>. } \\
\text { (Sélectionnez une réponse par ligne.) }\end{array}$} \\
\hline & & $\begin{array}{c}\begin{array}{c}\text { Ce n'est pas } \\
\text { arrivé }\end{array} \\
\text { and }\end{array}$ & $\begin{array}{l}1 \text { à } 2 \text { fois } \\
\text { dans } \\
\text { l'année }\end{array}$ & $\begin{array}{l}3 \text { à } 4 \text { fois } \\
\text { dans } \\
\text { l'année }\end{array}$ & $\begin{array}{l}\text { Une fois } \\
\text { par mois }\end{array}$ & $\begin{array}{l}\text { Une } \\
\text { fois par } \\
\text { semaine }\end{array}$ & $\begin{array}{l}\text { Plus d'une } \\
\text { fois par } \\
\text { semaine }\end{array}$ \\
\hline SC009Q01TA & $\begin{array}{l}\text { Je me sers des résultats des élèves } \\
\text { pour élaborer les objectifs pédagogiques } \\
\text { de l'établissement. }\end{array}$ & $\square_{1}$ & $\square_{2}$ & $\square_{3}$ & $\square_{4}$ & $\square_{5}$ & $\square_{6}$ \\
\hline SC009Q02TA & $\begin{array}{l}\text { Je fais en sorte que les activités } \\
\text { de formation continue des enseignants } \\
\text { soient en accord avec les objectifs } \\
\text { d'enseignement de l'établissement. }\end{array}$ & $\square_{1}$ & & $\square_{3}$ & $\square_{4}$ & $\square_{5}$ & $\square_{6}$ \\
\hline SC009Q03TA & $\begin{array}{l}\text { Je veille à ce que les enseignants } \\
\text { travaillent en accord avec les objectifs } \\
\text { d'enseignement de l'établissement. }\end{array}$ & $\square_{1}$ & $\square$ & $\square_{3}$ & $\square_{4}$ & $\square_{5}$ & $\square_{6}$ \\
\hline SC009Q04TA & $\begin{array}{l}\text { J'encourage les méthodes } \\
\text { d'enseignement fondées sur les résultats } \\
\text { de recherches récentes en éducation. }\end{array}$ & $\square_{1}$ & $\square_{2}$ & $\square_{3}$ & $\square_{4}$ & $\square_{5}$ & $\square_{6}$ \\
\hline SC009Q05TA & $\begin{array}{l}\text { Je complimente les enseignants dont les } \\
\text { élèves participent activement } \\
\text { aux activités d'apprentissage. }\end{array}$ & $\square_{1}$ & $\square_{2}$ & $\square_{3}$ & $\square_{4}$ & $\square_{5}$ & $\square_{6}$ \\
\hline SC009Q06TA & $\begin{array}{l}\text { Quand un professeur rencontre } \\
\text { un problème dans sa classe, je prends } \\
\text { l'initiative d'en discuter avec elle/lui. }\end{array}$ & $\square_{1}$ & $\square_{2}$ & $\square_{3}$ & $\square_{4}$ & $\square_{5}$ & $\square_{6}$ \\
\hline SC009Q07TA & $\begin{array}{l}\text { J'attire l'attention des enseignants } \\
\text { sur l'importance du développement } \\
\text { de l'esprit critique et du sens social } \\
\text { chez les élèves. }\end{array}$ & $\square_{1}$ & $\square_{2}$ & $\square_{3}$ & $\square_{4}$ & $\square_{5}$ & $\square_{6}$ \\
\hline SC009Q08TA & $\begin{array}{l}\text { Je suis attentif aux comportements qui } \\
\text { risquent de perturber le travail en classe. }\end{array}$ & $\square_{1}$ & $\square_{2}$ & $\square_{3}$ & $\square_{4}$ & $\square_{5}$ & $\square 6$ \\
\hline SC009Q09TA & $\begin{array}{l}\text { Je donne au personnel enseignant } \\
\text { la possibilité d'intervenir dans } \\
\text { les décisions concernant l'établissement. }\end{array}$ & $\square_{1}$ & $\square_{2}$ & $\square_{3}$ & $\square_{4}$ & $\square_{5}$ & $\square_{6}$ \\
\hline SC009Q10TA & $\begin{array}{l}\text { J'incite les enseignants à instaurer } \\
\text { une culture de l'établissement axée } \\
\text { sur l'amélioration continue. }\end{array}$ & $\square_{1}$ & $\square_{2}$ & $\square_{3}$ & $\square_{4}$ & $\square_{5}$ & $\square_{6}$ \\
\hline SC009Q11TA & $\begin{array}{l}\text { Je demande aux enseignants } \\
\text { de participer à l'évaluation des pratiques } \\
\text { de gestion. }\end{array}$ & $\square_{1}$ & $\square_{2}$ & $\square_{3}$ & $\square_{4}$ & $\square_{5}$ & $\square_{6}$ \\
\hline SC009Q12TA & $\begin{array}{l}\text { Quand un professeur évoque } \\
\text { un problème en classe, } \\
\text { nous le résolvons ensemble. }\end{array}$ & $\square_{1}$ & $\square_{2}$ & $\square_{3}$ & $\square_{4}$ & $\square_{5}$ & $\square_{6}$ \\
\hline SC009Q13TA & $\begin{array}{l}\text { Je discute des objectifs pédagogiques } \\
\text { de l'établissement avec les enseignants } \\
\text { lors de réunions pédagogiques. }\end{array}$ & $\square_{1}$ & $\square_{2}$ & $\square_{3}$ & $\square_{4}$ & $\square_{5}$ & $\square_{6}$ \\
\hline
\end{tabular}


Quelles sont les instances qui ont une part importante de responsabilité dans la gestion de votre établissement?

(À chaque ligne, sélectionnez toutes les réponses qui conviennent.)

\begin{tabular}{|c|c|c|c|c|c|c|}
\hline & & $\begin{array}{c}\text { Le chef } \\
\text { d'établissement }\end{array}$ & $\begin{array}{l}\text { Les } \\
\text { enseignants }\end{array}$ & $\begin{array}{c}\text { Le }<\text { Conseil } \\
\text { de direction } \\
\text { de } \\
\text { l'établissement }>\end{array}$ & \begin{tabular}{|c} 
Les <autorités \\
régionales \\
ou locales \\
responsables \\
de \\
l'éducation>
\end{tabular} & $\begin{array}{l} \\
\text { Les autorités } \\
\text { nationales } \\
\text { responsables } \\
\text { de l'éducation }\end{array}$ \\
\hline SC010Q01T & Choisir les enseignants à engager & $\square_{1}$ & $\square_{1}$ & $\square_{1}$ & $\square_{1}$ & $\square_{1}$ \\
\hline SC010Q02T & Congédier les enseignants & $\square_{1}$ & $\square_{1}$ & $\square_{1}$ & $\square_{1}$ & $\square_{1}$ \\
\hline SC010Q03T & $\begin{array}{l}\text { Déterminer le salaire initial } \\
\text { des enseignants }\end{array}$ & $\square_{1}$ & $\square_{1}$ & $\square_{1}$ & $\square_{1}$ & $\square_{1}$ \\
\hline SC010Q04T & $\begin{array}{l}\text { Déterminer les augmentations } \\
\text { de salaire des enseignants }\end{array}$ & $\square_{1}$ & $\square_{1}$ & $\square_{1}$ & $\square_{1}$ & $\square_{1}$ \\
\hline SC010Q05T & $\begin{array}{l}\text { Établir le budget de } \\
\text { I'établissement }\end{array}$ & $\square_{1}$ & $\square_{1}$ & $\square_{1}$ & $\square_{1}$ & $\square_{1}$ \\
\hline SC010Q06T & $\begin{array}{l}\text { Décider de la ventilation } \\
\text { du budget dans I'établissement }\end{array}$ & $\square_{1}$ & $\square_{1}$ & $\square_{1}$ & $\square_{1}$ & $\square_{1}$ \\
\hline SC010Q07T & $\begin{array}{l}\text { Définir le règlement intérieur } \\
\text { pour les élèves }\end{array}$ & $\square_{1}$ & $\square_{1}$ & $\square_{1}$ & $\square_{1}$ & $\square_{1}$ \\
\hline SC010Q08T & $\begin{array}{l}\text { Définir les politiques d'évaluation } \\
\text { des élèves }\end{array}$ & $\square_{1}$ & $\square_{1}$ & $\square_{1}$ & $\square_{1}$ & $\square_{1}$ \\
\hline SC010Q09T & $\begin{array}{l}\text { Décider de l'admission des élèves } \\
\text { dans l'établissement }\end{array}$ & $\square_{1}$ & $\square_{1}$ & $\square_{1}$ & $\square_{1}$ & $\square_{1}$ \\
\hline SC010Q10T & Choisir les manuels à utiliser & $\square_{1}$ & $\square_{1}$ & $\square_{1}$ & $\square_{1}$ & $\square_{1}$ \\
\hline SC010Q11T & Déterminer le contenu des cours & $\square_{1}$ & $\square_{1}$ & $\square_{1}$ & $\square_{1}$ & $\square_{1}$ \\
\hline SC010Q12T & Décider des cours à proposer & $\square_{1}$ & $\square$ & $\square_{1}$ & $\square_{1}$ & $\square_{1}$ \\
\hline
\end{tabular}

SC012

Dans quelle mesure tient-on compte des facteurs suivants pour admettre un élève dans votre établissement ?

(Sélectionnez une réponse par ligne.)

\begin{tabular}{|c|c|c|c|c|}
\hline & & Jamais & Parfois & Toujours \\
\hline SC012Q01TA & $\begin{array}{l}\text { Dossier scolaire de l'élève avec ses résultats (y compris des tests } \\
\text { de placement) }\end{array}$ & $\square_{1}$ & $\square_{2}$ & $\square_{3}$ \\
\hline SC012Q02TA & Recommandation de l'établissement dont provient l'élève & $\square_{1}$ & $\square_{2}$ & $\square_{3}$ \\
\hline SC012Q03TA & $\begin{array}{l}\text { Adhésion des parents à la " philosophie " pédagogique ou religieuse } \\
\text { de l'établissement }\end{array}$ & $\square_{1}$ & $\square_{2}$ & $\square_{3}$ \\
\hline SC012Q04TA & $\begin{array}{l}\text { Le fait que l'élève doive suivre un programme scolaire spécifique } \\
\text { ou s'y intéresse }\end{array}$ & $\square_{1}$ & $\square_{2}$ & $\square_{3}$ \\
\hline SC012Q05TA & $\begin{array}{l}\text { Priorité accordée aux membres de la famille d'un élève fréquentant } \\
\text { ou ayant fréquenté l'établissement }\end{array}$ & $\square_{1}$ & $\square_{2}$ & $\square_{3}$ \\
\hline SC012Q06TA & Domicile dans une entité géographique déterminée & $\square_{1}$ & $\square_{2}$ & $\square_{3}$ \\
\hline SC012Q07TA & Autres & $\square_{1}$ & $\square_{2}$ & $\square_{3}$ \\
\hline
\end{tabular}

SC013 SC013Q01TA
Votre établissement est-il un établissement public ou privé ?

(Sélectionnez une réponse.)

Un établissement public

(Il s'agit dans ce cas d'un établissement relevant et dépendant directement ou indirectement d'instances publiques en charge de l'éducation, d'un organisme gouvernemental ou dont la direction est désignée/nommée par les pouvoirs publics.)

Un établissement privé

(Il s'agit dans ce cas d'un établissement relevant et dépendant directement ou indirectement d'un organisme non gouvernemental, par exemple une congrégation religieuse, un syndicat, une entreprise ou autre institution privée.)

Il s'agit ici d'une question filtre.

Si l'établissement est privé (réponse à la question SC013Q01TA : "Un établissement privé », aller à la question SC014Q01NA. Sinon, passer directement à la question SC016. 
SC014

SC014Q01NA

Il s'agit ici d'une question filtrée :

Ne répondre que si vous avez choisi l'option de réponse « Un établissement privé » à la question SC013Q01TA. Sinon, passer directement à la question SC016.

\section{Quel type d'organisation dirige votre établissement ?}

(Sélectionnez une réponse.)

\begin{tabular}{|l|c|} 
Une congrégation religieuse ou une autre organisation religieuse & $\square_{1}$ \\
\hline Une autre organisation à but non lucratif & $\square_{2}$ \\
\hline Une organisation à but lucratif & $\square_{3}$
\end{tabular}

SC016

Au cours d'une année scolaire ordinaire, quel est le pourcentage approximatif de votre budget total qui provient des sources suivantes?

(Tapez un nombre par ligne. Tapez « 0 » (zéro) s'il n'y en a aucun.)

\begin{tabular}{|c|c|c|}
\hline & & $\%$ \\
\hline SC016Q01TA & Des pouvoirs publics (qu'ils soient municipaux, départementaux, régionaux, nationaux ou fédéraux) & \\
\hline SC016Q02TA & Des droits d'inscription et frais scolaires payés par les parents & \\
\hline SC016Q03TA & De dons, de legs, de parrainages, de collectes de fonds réalisées par les parents & \\
\hline \multirow[t]{2}{*}{ SC016Q04TA } & D'autres sources & \\
\hline & Total & $100 \%$ \\
\hline
\end{tabular}

L'enseignement que votre établissement est à même de dispenser est-il affecté par les problèmes suivants ?

(Sélectionnez une réponse par ligne.)

\begin{tabular}{|c|c|c|c|c|c|}
\hline & & Pas du tout & Très peu & $\begin{array}{l}\text { Dans une } \\
\text { certaine } \\
\text { mesure }\end{array}$ & Beaucoup \\
\hline SC017Q01NA & Manque de personnel enseignant & $\square_{1}$ & $\square_{2}$ & $\square_{3}$ & $\square_{4}$ \\
\hline SC017Q02NA & Personnel enseignant inadéquat ou peu qualifié & $\square_{1}$ & $\square_{2}$ & $\square_{3}$ & $\square_{4}$ \\
\hline SC017Q03NA & Manque de personnel auxiliaire & $\square_{1}$ & $\square_{2}$ & $\square_{3}$ & $\square_{4}$ \\
\hline SC017Q04NA & Personnel auxiliaire inadéquat ou peu qualifié & $\square_{1}$ & $\square_{2}$ & $\square_{3}$ & $\square_{4}$ \\
\hline SC017Q05NA & $\begin{array}{l}\text { Manque de matériel pédagogique (par ex. manuels } \\
\text { scolaires, équipement informatique, matériel } \\
\text { de bibliothèque ou de laboratoire) }\end{array}$ & $\square_{1}$ & $\square_{2}$ & $\square_{3}$ & $\square_{4}$ \\
\hline SC017Q06NA & $\begin{array}{l}\text { Matériel pédagogique inadéquat ou de mauvaise } \\
\text { qualité (par ex. manuels scolaires, équipement } \\
\text { informatique, matériel de bibliothèque ou } \\
\text { de laboratoire) }\end{array}$ & $\square_{1}$ & $\square_{2}$ & $\square_{3}$ & $\square_{4}$ \\
\hline SC017Q07NA & $\begin{array}{l}\text { Manque d'infrastructures (par ex. bâtiments, terrain, } \\
\text { chauffage/climatisation, systèmes d'éclairage et } \\
\text { acoustiques) }\end{array}$ & $\square_{1}$ & $\square_{2}$ & $\square_{3}$ & $\square_{4}$ \\
\hline SC017Q08NA & $\begin{array}{l}\text { Infrastructures inadéquates ou de mauvaise qualité } \\
\text { (par ex. bâtiments, terrain, chauffage/ climatisation, } \\
\text { systèmes d'éclairage et acoustiques) }\end{array}$ & $\square_{1}$ & $\square_{2}$ & $\square_{3}$ & $\square_{4}$ \\
\hline
\end{tabular}




\section{Le personnel enseignant}

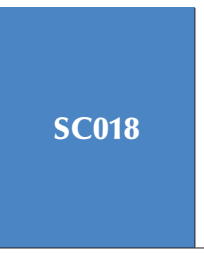

Dans votre établissement, combien d'enseignants y a-t-il dans chacune des catégories suivantes ?

Comptez séparément les enseignants à temps plein et à temps partiel. Un enseignant est considéré "à temps plein " s'il consacre au moins $90 \%$ d'un emploi du temps complet à l'enseignement en salle de classe, et ce tout au long de l'année scolaire. Tous les autres enseignants doivent être considérés "à temps partiel ». En ce qui concerne les qualifications, ne prenez en compte que le niveau de formation le plus élevé des enseignants.

(Tapez un nombre dans chaque espace disponible. Tapez " 0 » (zéro) s'il n'y en a pas.)

\begin{tabular}{|c|c|c|c|}
\hline & & $\begin{array}{l}\text { À temps } \\
\text { plein }\end{array}$ & $\begin{array}{l}\text { À temps } \\
\text { partiel }\end{array}$ \\
\hline SC018Q01TA & Enseignants au TOTAL & & \\
\hline SC018Q02TA & $\begin{array}{l}\text { Enseignants ayant des titres donnant accès à la fonction d'enseignant qui sont } \\
<\text { pleinement reconnus }>\text { par }<\text { l'autorité de tutelle }>\end{array}$ & & \\
\hline SC018Q05NA & Enseignants possédant un diplôme de $<$ Niveau $5 \mathrm{~A}$ de la CITE Bachelier/licence $>$ & & \\
\hline SC018Q06NA & Enseignants possédant un diplôme de < Niveau 5A de la CITE Master $>$ & & \\
\hline SC018Q07NA & Enseignants possédant un diplôme de $<$ Niveau 6 de la CITE $>$ & & \\
\hline
\end{tabular}

Dans votre établissement, combien d'enseignants de <cours de sciences $>$ y a-t-il dans chacune des catégories suivantes?

\section{(10}

Comptez séparément les enseignants à temps partiel et à temps plein. Un enseignant est considéré "à temps plein » s'il consacre au moins $90 \%$ d'un emploi du temps complet à l'enseignement en salle de classe, et ce tout au long de l'année scolaire. Tous les autres enseignants doivent être considérés "à temps partiel ».

(Tapez un nombre dans chaque espace disponible. Tapez " 0 » (zéro) s'il n'y en a pas.)

\begin{tabular}{|c|c|c|c|}
\hline & & $\begin{array}{l}\text { À temps } \\
\text { plein }\end{array}$ & $\begin{array}{l}\text { À temps } \\
\text { partiel }\end{array}$ \\
\hline SC019Q01NA & Enseignants de $<$ cours de sciences $>$ au TOTAL & & \\
\hline SC019Q02NA & $\begin{array}{l}\text { Enseignants de }<\text { cours de sciences }>\text { ayant des titres donnant accès à la fonction } \\
\text { d'enseignant qui sont }<\text { pleinement reconnus }>\text { par }<\text { l'autorité de tutelle }>\end{array}$ & & \\
\hline SC019Q03NA & $\begin{array}{l}\text { Enseignants de }<\text { cours de sciences }>\text { possédant un diplôme de }<\text { Niveau } 5 \text { A de la CITE } \\
\text { ou supérieur }><\text { avec une spécialisation }>\text { en }<\text { cours de sciences }>\end{array}$ & & \\
\hline
\end{tabular}

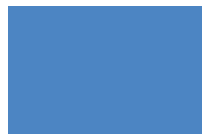

Au cours des trois derniers mois, quel est le pourcentage d'enseignants dans votre établissement ayant participé à un programme de formation continue ?

Par formation continue, on entend un programme officiel, destiné à améliorer les compétences ou les pratiques pédagogiques des enseignants. Ce programme peut être sanctionné par une certification reconnue ou non. La durée totale du programme doit être d'au moins une journée, et doit porter sur la pédagogie et l'enseignement. (Positionnez le curseur sur le pourcentage correspondant. Positionnez le curseur sur « 0 » (zéro) si aucun de vos enseignants n'a participé à des activités de formation continue.)

SC025Q01NA Sur l'ensemble du personnel enseignant de votre établissement

\begin{tabular}{l|l} 
SC025Q02NA & Enseignants de sciences de l'établissement
\end{tabular}

Curseur : position initiale, plage $« 0-100 »$; graduation $=1$.

\begin{tabular}{c|l|c|c}
\multicolumn{1}{c|}{} & Oui & Non \\
\hline SC027Q01NA & $\begin{array}{l}\text { Les enseignants de notre établissement coopèrent en échangeant des idées ou } \\
\text { du matériel sur certains modules de cours ou certaines leçons en particulier. }\end{array}$ & $\square_{1}$ & $\square_{2}$ \\
\hline SC027Q02NA & $\begin{array}{l}\text { Notre établissement invite des spécialistes pour donner des formations } \\
\text { à nos enseignants, sur leur lieu de travail. }\end{array}$ & $\square_{1}$ & $\square_{2}$ \\
\hline SC027Q03NA & $\begin{array}{l}\text { Notre établissement organise des ateliers sur le lieu de travail abordant } \\
\text { des problèmes particuliers rencontrés par l'établissement. }\end{array}$ & $\square_{1}$ & $\square_{2}$ \\
\hline SC027Q04NA & $\begin{array}{l}\text { Notre établissement organise des ateliers sur le lieu de travail pour des groupes } \\
\text { d'enseignants spécifiques (par ex. les enseignants récemment affectés). }\end{array}$ & $\square_{1}$ & $\square_{2}$ \\
\hline
\end{tabular}




\section{L'évaluation}

\begin{tabular}{c|l|c}
\multicolumn{1}{c|}{ SC032 } & $\begin{array}{l}\text { Au cours de <l'année scolaire écoulée>, a-t-on utilisé les méthodes suivantes pour contrôler } \\
\text { les pratiques des enseignants dans votre établissement ? } \\
\text { (Sélectionnez une réponse par ligne.) }\end{array}$ & \multicolumn{1}{c}{ Oui } \\
\hline & & $\square_{1}$ \\
\hline SC032Q01TA & Tests ou contrôles évaluant les performances des élèves & $\square_{2}$ \\
\hline SC032Q02TA & $\begin{array}{l}\text { Évaluation mutuelle entre collègues (passer en revue des plans de cours, } \\
\text { des instruments d'évaluation, des leçons) }\end{array}$ & $\square_{1}$ \\
\hline SC032Q03TA & Observation des cours par le chef d'établissement ou par des enseignants chevronnés & $\square_{2}$ \\
\hline SC032Q04TA & $\begin{array}{l}\text { Observation des cours par des inspecteurs ou par d'autres personnes extérieures } \\
\text { à l'établissement }\end{array}$ & $\square_{2}$ \\
\hline
\end{tabular}

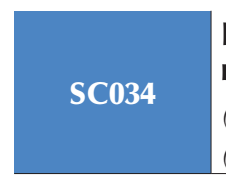

D'habitude, dans votre établissement, à quelle fréquence évalue-t-on les élèves de < grade modal du pays pour les élèves de $\mathbf{1 5}$ ans $>$ au moyen des méthodes suivantes ?

(Si vous avez besoin d'explications au sujet du terme <tests standardisés>, utilisez le bouton d'aide.) (Sélectionnez une réponse par ligne.)

\begin{tabular}{|c|c|c|c|c|c|c|}
\hline & & Jamais & $\begin{array}{l}1 \text { ou } 2 \text { fois } \\
\text { par an }\end{array}$ & $\begin{array}{l}3 \text { à } 5 \text { fois } \\
\text { par an }\end{array}$ & $\begin{array}{l}\text { Une fois } \\
\text { par mois }\end{array}$ & $\begin{array}{l}\text { Plus d'une fois } \\
\text { par mois }\end{array}$ \\
\hline SC034Q01NA & $\begin{array}{l}\text { Des <tests standardisés }>\text { obligatoires, comme } \\
<\text { exemple spécifique au pays }>\end{array}$ & $\square_{1}$ & $\square_{2}$ & $\square_{3}$ & & \\
\hline SC034Q02NA & $\begin{array}{l}\text { Des <tests standardisés }>\text { non obligatoires } \\
\text { (par ex. accessibles au public ou disponibles } \\
\text { dans le commerce, comme < exemple spécifique } \\
\text { au pays }>\text { ) }\end{array}$ & $\square_{1}$ & & & & \\
\hline SC034Q03TA & Des épreuves mises au point par les enseignants & $\square_{1}$ & $\square_{2}$ & $\square_{3}$ & $\square_{4}$ & $\square_{5}$ \\
\hline SC034Q04TA & $\begin{array}{l}\text { Des appréciations informelles fournies } \\
\text { par les enseignants }\end{array}$ & $\square_{1}$ & $\square_{2}$ & $\square_{3}$ & $\square_{4}$ & $\square_{5}$ \\
\hline
\end{tabular}

Bouton d'aide $\quad$ Les <tests standardisés> présentent une cohérence dans leur conception, leur contenu, leur administration et leur système de notation. Les résultats peuvent être comparés entre élèves et entre établissements.

Il s'agit ici d'une question filtre.

Si la réponse choisie pour SC034Q01NA, SC034Q02NA ou SC034Q03TA est supérieure à l'option de réponse 1 (" Jamais »), répondre à la question SC035.

Dans le cas contraire, passer la question sur l'utilisation des résultats des élèves aux tests et aller directement à la question SC036. 


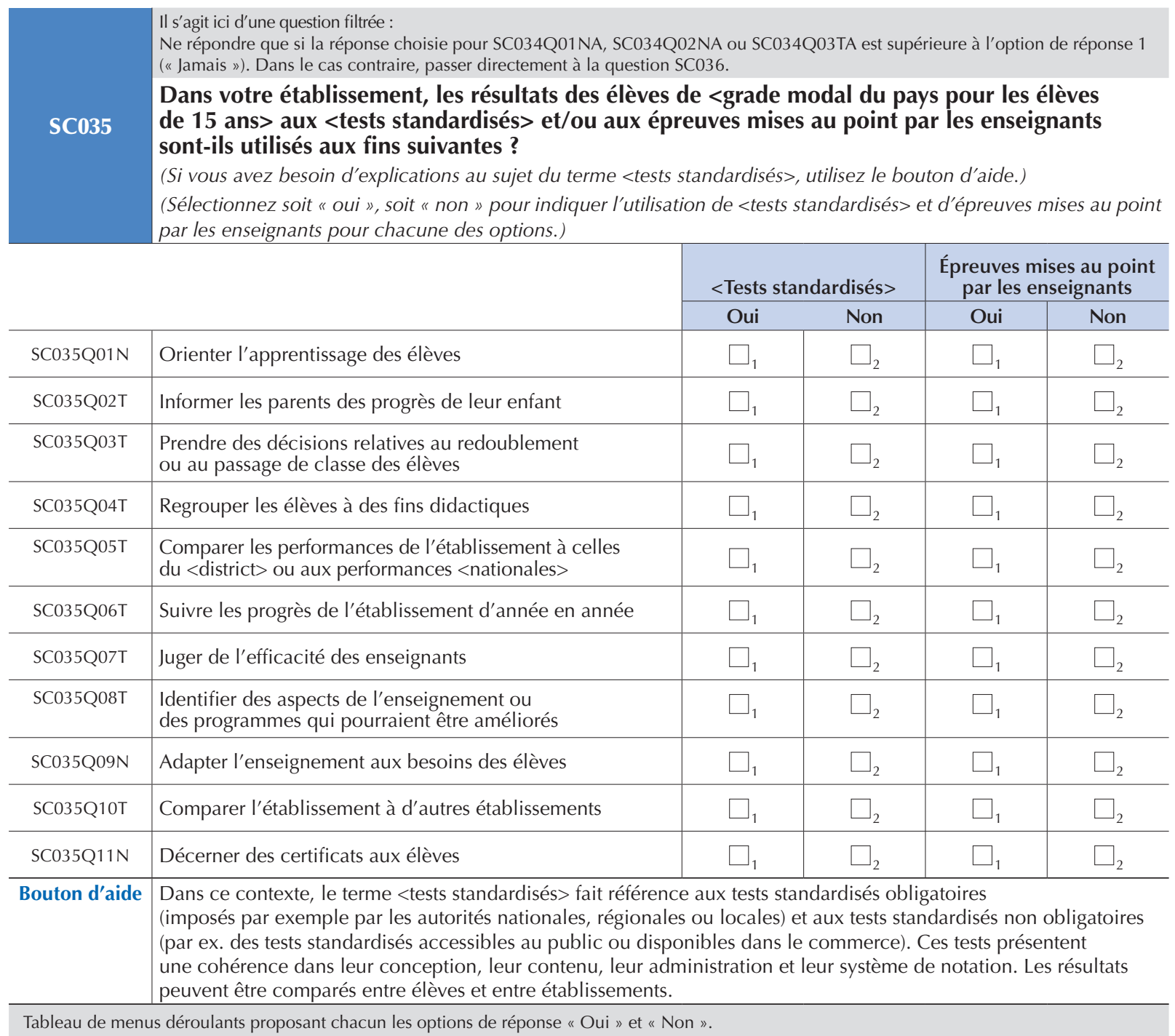

\begin{tabular}{|c|c|c|c|}
\hline \multirow[t]{2}{*}{ SC036 } & \multicolumn{3}{|c|}{$\begin{array}{l}\text { Dans votre établissement, les données relatives aux résultats scolaires des élèves sont-elles } \\
\text { utilisées dans le cadre d'un des <mécanismes de pilotage> suivants? } \\
\text { Par « données relatives aux résultats scolaires », on entend notamment des données agrégées (les moyennes de résultats } \\
\text { d'examen ou de notes, ou bien les taux de réussite), calculées à l'échelle de l'établissement ou d'un grade donné } \\
\text { (Sélectionnez une réponse par ligne.) }\end{array}$} \\
\hline & & Oui & Non \\
\hline SC036Q01TA & $\begin{array}{l}\text { Les données relatives aux résultats scolaires sont rendues publiques (par exemple } \\
\text { dans les médias). }\end{array}$ & & \\
\hline SC036Q02TA & $\begin{array}{l}\text { Les données relatives aux résultats scolaires font l'objet d'un suivi au cours du temps } \\
\text { par une instance administrative. }\end{array}$ & $\square_{1}$ & \\
\hline SC036Q03NA & Les données relatives aux résultats scolaires sont fournies directement aux parents. & $\square_{1}$ & $\square_{2}$ \\
\hline
\end{tabular}


Les dispositions suivantes visant à garantir et à améliorer la qualité existent-elles dans votre établissement, et quelle est leur origine?

Si vous avez besoin d'explications au sujet des termes "évaluation interne » ou " évaluation externe », utilisez le bouton d'aide.

(Sélectionnez une réponse par ligne.)

\begin{tabular}{|c|c|c|c|c|}
\hline & & ou locales & sement & Non \\
\hline SC037Q01TA & Évaluation interne/auto-évaluation & $\square_{1}$ & $\square_{2}$ & $\square_{3}$ \\
\hline SC037Q02TA & Évaluation externe & $\square_{1}$ & $\square_{2}$ & $\square_{3}$ \\
\hline SC037Q03TA & $\begin{array}{l}\text { Descriptif écrit du programme scolaire et des objectifs pédagogiques } \\
\text { de l'établissement }\end{array}$ & $\square_{1}$ & $\square_{2}$ & $\square_{3}$ \\
\hline SC037Q04TA & $\begin{array}{l}\text { Descriptif écrit des normes requises en termes de performances } \\
\text { des élèves }\end{array}$ & $\square_{1}$ & & $\square_{3}$ \\
\hline SC037Q05NA & $\begin{array}{l}\text { Enregistrement systématique de données comme les taux de présence } \\
\text { des enseignants et des élèves et la formation continue des enseignants }\end{array}$ & $\square_{1}$ & & $J_{3}$ \\
\hline SC037Q06NA & $\begin{array}{l}\text { Enregistrement systématique des résultats des élèves aux évaluations } \\
\text { et des taux de réussite }\end{array}$ & $\square_{1}$ & $\square_{2}$ & $\square_{3}$ \\
\hline SC037Q07TA & $\begin{array}{l}\text { Demande d'un retour écrit de la part des élèves (par ex. par rapport } \\
\text { aux cours, aux enseignants ou aux ressources) }\end{array}$ & $\square_{1}$ & $\square_{2}$ & $\square_{3}$ \\
\hline SC037Q08TA & Tutorat des enseignants & $\square_{1}$ & $\square_{2}$ & $\square_{3}$ \\
\hline SC037Q09TA & $\begin{array}{l}\text { Projet d'amélioration de l'établissement en concertation } \\
\text { avec un ou plusieurs experts pendant six mois au moins }\end{array}$ & $\square_{1}$ & $\square_{2}$ & $\square_{3}$ \\
\hline SC037Q10NA & $\begin{array}{l}\text { Mise en œuvre d'une politique normalisée d'enseignement } \\
\text { des sciences (le programme de cours est mis en œuvre avec } \\
\text { du matériel pédagogique commun, ainsi que la formation continue } \\
\text { du personnel enseignant) }\end{array}$ & $\square_{1}$ & $\square_{2}$ & $\square_{3}$ \\
\hline
\end{tabular}

Bouton d'aide Évaluation interne de l'établissement : cette évaluation fait partie d'un processus dépendant de l'établissement, qui définit quels éléments sont évalués. Elle peut être menée par des membres de cet établissement ou par des personnes/institutions mandatées par l'établissement.

Bouton d'aide Évaluation externe : cette évaluation fait partie d'un processus contrôlé et dirigé par un organisme externe. L'établissement ne détermine pas les domaines à évaluer.

Il s'agit ici d'une question filtre.

Si l'option de réponse choisie pour la question SC037Q01TA est « Oui, c'est obligatoire et émane par ex. de politiques ministérielles ou locales » ou "Oui, à l'initiative de l'établissement », passer aux questions complémentaires relatives aux dispositifs d'évaluation interne (SC040).

Si l'option de réponse choisie pour la question SC037Q02TA est «Oui, c'est obligatoire et émane par ex. de politiques ministérielles ou locales » ou "Oui, à l'initiative de l'établissement », passer aux questions complémentaires relatives aux dispositifs d'évaluation externe (SC041).

Dans le cas contraire, passer toutes les questions relatives aux dispositifs d'évaluation et aller directement à la question SC042. 


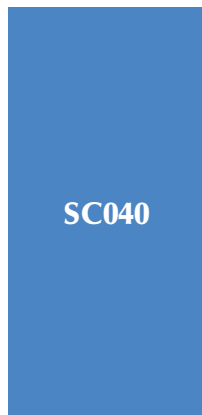

Il s'agit ici d'une question filtrée :

Ne répondre que si vous avez choisi l'option de réponse « Oui, c'est obligatoire et émane par ex. de politiques ministérielles ou locales » ou "Oui, à l'initiative de l'établissement » à la question SC037Q01TA.

Si vous avez choisi l'option de réponse "Oui, c'est obligatoire et émane par ex. de politiques ministérielles ou locales » ou " Oui, à l'initiative de l'établissement » à la question SC037Q02TA, passer à la question relative aux dispositifs d'évaluation externe (SC041).

Dans le cas contraire, passer directement à la question SC042.

Sur la base des résultats de votre dernière évaluation interne, votre établissement a-t-il appliqué des mesures dans les domaines suivants?

Si vous avez besoin d'explications au sujet du terme "évaluation interne », utilisez le bouton d'aide.

(Sélectionnez une réponse par ligne.)

\begin{tabular}{|c|c|c|c|c|}
\hline & & Oui & satisfaisants & raisons \\
\hline SC040Q02NA & $\begin{array}{l}\text { Le personnel enseignant (par ex. charge de travail, exigences } \\
\text { individuelles, qualifications) }\end{array}$ & $\square_{1}$ & & \\
\hline SC040Q03NA & La mise en œuvre du programme de cours & $\square_{1}$ & $\square_{2}$ & $\square_{3}$ \\
\hline SC040Q05NA & La qualité de I'enseignement et de l'apprentissage & $\square_{1}$ & $\square_{2}$ & $\square_{3}$ \\
\hline SC040Q11NA & L'implication des parents dans l'établissement & $\square_{1}$ & $\square_{2}$ & $\square_{3}$ \\
\hline SC040Q12NA & La formation continue des enseignants & $\square_{1}$ & $\square_{2}$ & $\square_{3}$ \\
\hline SC040Q15NA & La réussite des élèves & $\square_{1}$ & $\square_{2}$ & $\square_{3}$ \\
\hline SC040Q16NA & Les compétences transversales des élèves & $\square_{1}$ & $\square_{2}$ & $\square_{3}$ \\
\hline SC040Q17NA & L'équité dans l'établissement & $\square_{1}$ & $\square_{2}$ & $\square_{3}$ \\
\hline Bouton d'aide & \multicolumn{4}{|c|}{$\begin{array}{l}\text { Évaluation interne de l'établissement : cette évaluation fait partie d'un processus dépendant de l'établissement, } \\
\text { qui définit quels éléments sont évalués. Elle peut être menée par des membres de cet établissement ou par des } \\
\text { personnes/institutions mandatées par l'établissement. }\end{array}$} \\
\hline
\end{tabular}

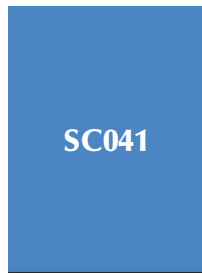

Il s'agit ici d'une question filtrée

Ne répondre que si vous avez choisi l'option de réponse «Oui, c'est obligatoire et émane par ex. de politiques ministérielles ou locales» ou « Oui, à l'initiative de l'établissement » à la question SC037Q02TA.

Dans le cas contraire, passer directement à la question SC042.

Les affirmations suivantes s'appliquent-elles à la dernière évaluation externe de votre établissement ?

Si vous avez besoin d'explications au sujet du terme " évaluation externe », utilisez le bouton d'aide.

(Sélectionnez une réponse par ligne.)

\begin{tabular}{|c|c|c|c|}
\hline & & Oui & Non \\
\hline SC041Q01NA & $\begin{array}{l}\text { Les résultats des évaluations externes ont entraîné des modifications } \\
\text { dans les politiques menées par l'établissement. }\end{array}$ & & \\
\hline SC041Q03NA & $\begin{array}{l}\text { Nous avons utilisé les données de l'évaluation pour mettre en place un plan d'action } \\
\text { spécifique pour le développement scolaire. }\end{array}$ & & \\
\hline SC041Q04NA & $\begin{array}{l}\text { Nous avons utilisé les données de l'évaluation pour mettre en place un plan d'action } \\
\text { spécifique pour faire progresser l'enseignement. }\end{array}$ & & \\
\hline SC041Q05NA & $\begin{array}{l}\text { Nous avons appliqué rapidement les mesures inspirées par les résultats } \\
\text { des évaluations externes. }\end{array}$ & $\square_{1}$ & \\
\hline SC041Q06NA & $\begin{array}{l}\text { L'élan suscité par la dernière évaluation externe est vite « retombé » } \\
\text { dans notre établissement. }\end{array}$ & $\square_{1}$ & \\
\hline
\end{tabular}

Bouton d'aide Évaluation externe : cette évaluation fait partie d'un processus contrôlé et dirigé par un organisme externe. L'établissement ne détermine pas les domaines à évaluer. 


\section{Groupes cibles}

Certains établissements organisent l'enseignement de manière différente en fonction des niveaux d'aptitude des élèves.

\begin{tabular}{|c|c|c|c|c|}
\hline \multirow[t]{2}{*}{ SC042 } & \multicolumn{4}{|c|}{$\begin{array}{l}\text { Quelle est la politique de votre établissement à cet égard, pour les élèves du <grade modal } \\
\text { du pays pour les élèves de } \mathbf{1 5} \text { ans>? } \\
\text { (Sélectionnez une réponse par ligne.) }\end{array}$} \\
\hline & & \begin{tabular}{|c|}
$\begin{array}{c}\text { Pour } \\
\text { toutes } \\
\text { les } \text { matières }\end{array}$ \\
\end{tabular} & $\begin{array}{l}\text { Pour } \\
\text { certaines } \\
\text { matières }\end{array}$ & $\begin{array}{c}\text { Pour } \\
\text { aucune } \\
\text { matière }\end{array}$ \\
\hline SC042Q01TA & $\begin{array}{l}\text { Les élèves sont répartis dans des classes différentes en fonction } \\
\text { de leur niveau d'aptitude. }\end{array}$ & $\square_{1}$ & $\square_{2}$ & $\square_{3}$ \\
\hline SC042Q02TA & $\begin{array}{l}\text { Les élèves sont répartis en divers groupes de niveau au sein } \\
\text { de leur classe en fonction de leurs aptitudes. }\end{array}$ & $\square_{1}$ & $\square_{2}$ & $\square_{3}$ \\
\hline
\end{tabular}

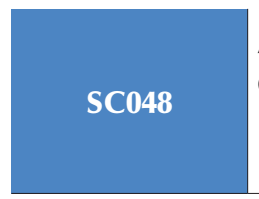

À combien estimez-vous le pourcentage d'élèves de cet établissement en < grade modal du pays pour les élèves de 15 ans $>$ qui présentent les caractéristiques suivantes ?

(Les élèves peuvent appartenir à plus d'une catégorie.)

(Positionnez le curseur sur le pourcentage correspondant.)

\begin{tabular}{|c|c|c|c|}
\hline & & $0 \%$ & $100 \%$ \\
\hline SC048Q01NA & Élèves dont la <langue d'origine > diffère de la <langue de l'évaluation > & \multicolumn{2}{|c|}{ 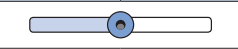 } \\
\hline SC048Q02NA & Élèves présentant des besoins éducatifs particuliers & \multicolumn{2}{|c|}{ 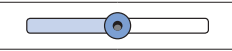 } \\
\hline SC048Q03NA & Élèves issus d'un milieu socio-économique défavorisé & \multicolumn{2}{|c|}{$\square$} \\
\hline
\end{tabular}

\section{Le climat de l'établissement}

Dans votre établissement, dans quelle mesure l'apprentissage des élèves est-il gêné par les facteurs suivants?

(Sélectionnez une réponse par ligne.)

\begin{tabular}{l|l|l|l}
\multicolumn{1}{l|}{} & $\begin{array}{c}\text { Pas du } \\
\text { tout }\end{array}$ & $\begin{array}{c}\text { Très } \\
\text { peu }\end{array}$ & $\begin{array}{c}\text { Dans une } \\
\text { certaine } \\
\text { mesure }\end{array}$ \\
Beaucoup
\end{tabular}


Les affirmations suivantes à propos de l'implication des parents s'appliquent-elles à votre établissement ?

(Sélectionnez une réponse par ligne.)

\begin{tabular}{|c|c|c|c|}
\hline & & Oui & Non \\
\hline SC063Q02NA & L'ambiance conviviale et ouverte de notre établissement favorise l'implication des parents. & & \\
\hline SC063Q03NA & $\begin{array}{l}\text { Notre établissement a mis au point une communication bilatérale efficace entre } \\
\text { l'établissement et les parents sur les programmes scolaires et les progrès des élèves. }\end{array}$ & & \\
\hline SC063Q04NA & Cet établissement implique les parents dans ses prises de décision. & & \\
\hline SC063Q06NA & $\begin{array}{l}\text { Notre établissement fournit des informations et des idées aux familles pour aider les élèves } \\
\text { dans leurs devoirs à la maison et sur d'autres activités, décisions et projets concernant } \\
\text { le programme. }\end{array}$ & 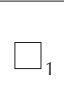 & \\
\hline SC063Q07NA & $\begin{array}{l}\text { Notre établissement identifie puis intègre des ressources et des services de la collectivité } \\
\text { pour renforcer le programme scolaire, les pratiques familiales, ainsi que l'apprentissage } \\
\text { et le développement des élèves. }\end{array}$ & $\square_{1}$ & 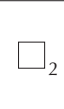 \\
\hline SC063Q09NA & $\begin{array}{l}\text { Une <législation nationale, régionale ou locale> prévoit l'implication des parents } \\
\text { dans les activités de l'établissement. }\end{array}$ & $\square_{1}$ & \\
\hline
\end{tabular}

\begin{tabular}{|c|c|c|}
\hline \multirow[t]{2}{*}{ SC064 } & \multicolumn{2}{|c|}{$\begin{array}{l}\text { Au cours de <l'année scolaire écoulée>, quel est le pourcentage de parents d'élèves ayant pris } \\
\text { part aux activités suivantes en rapport avec l'établissement? } \\
\text { (Positionnez le curseur sur le pourcentage correspondant. Positionnez-le sur " } 0 \text { » (zéro) si aucun parent n'a participé } \\
\text { à l'activité. Positionnez-le sur " } 100 \text { » (cent) si tous les parents ont participé à cette activité) }\end{array}$} \\
\hline & & $\%$ \\
\hline SC064Q01TA & Discuter des progrès de leur enfant avec un enseignant, sur leur initiative & $(0$ \\
\hline SC064Q02TA & Discuter des progrès de leur enfant avec un enseignant, sur l'initiative de ce dernier & 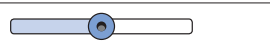 \\
\hline SC064Q03TA & $\begin{array}{l}\text { Participer à des activités liées à la gestion de l'établissement (par ex. faire partie } \\
\text { d'un comité consultatif de parents d'élèves ou du comité de direction } \\
\text { de l'établissement) }\end{array}$ & 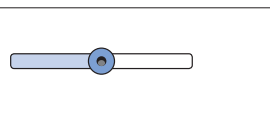 \\
\hline SC064Q04NA & $\begin{array}{l}\text { Se porter volontaires pour des tâches manuelles ou des activités parascolaires } \\
\text { (par ex. entretien des bâtiments, travaux de menuiserie, travaux de jardinage, } \\
\text { pièce de théâtre, sports, excursion) }\end{array}$ & 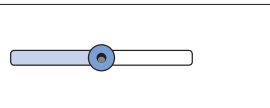 \\
\hline
\end{tabular}




\section{QUESTIONNAIRE ÉL̇̀VE}

\section{Version en ligne/version source pour la campagne définitive}

Le questionnaire "Élève » est administré après les épreuves cognitives. Environ 35 minutes sont nécessaires pour le remplir.

Les questions sur le milieu familial sont similaires à celles utilisées lors des enquêtes PISA précédentes. Ce questionnaire est destiné à recueillir des renseignements sur :

- L'élève, sa famille et son environnement à la maison

- Ce qu'il pense de sa vie

- Son établissement

- Son horaire scolaire et le temps consacré à apprendre

- Les <cours de sciences> dans son établissement

- Son avis sur les sciences

Les termes techniques sont indiqués entre <crochets> et adaptés au contexte national par le centre national de collecte de données des pays ou économies participants. Dans cette annexe, ces termes techniques sont expliqués sous les items.

\section{Vous, votre famille et votre environnement à la maison}

ST001

ST001Q01TA

Quel < grade> fréquentez-vous ?

(Sélectionnez votre réponse dans le menu déroulant.)

\begin{tabular}{|l|}
\hline Sélectionnez une option $\mathbf{\nabla}$ \\
\hline Option A \\
Option B \\
Option C \\
Option ...
\end{tabular}

Menu déroulant incluant tous les < grades> pouvant être fréquentés par des élèves de 15 ans conformément au tableau des programmes

d'enseignement, comme établi dans les tâches démographiques.

ST002

ST002Q01TA
Lequel de ces <programmes> suivez-vous?

(Sélectionnez une réponse.)

\begin{tabular}{l|c}
\hline <Programme 1> & $\square_{1}$ \\
\hline$<$ Programme 2> & $\square_{2}$ \\
\hline$<$ Programme 3> & $\square_{3}$ \\
\hline$<$ Programme 4> & $\square_{4}$ \\
\hline$<$ Programme 5> & $\square_{5}$ \\
\hline$<$ Programme 6> & $\square_{6}$ \\
\hline
\end{tabular}




\section{ST003}

Quelle est votre date de naissance ?

(Pour répondre à la question, sélectionnez le jour, le mois et l'année de votre naissance dans les menus déroulants ci dessous.)

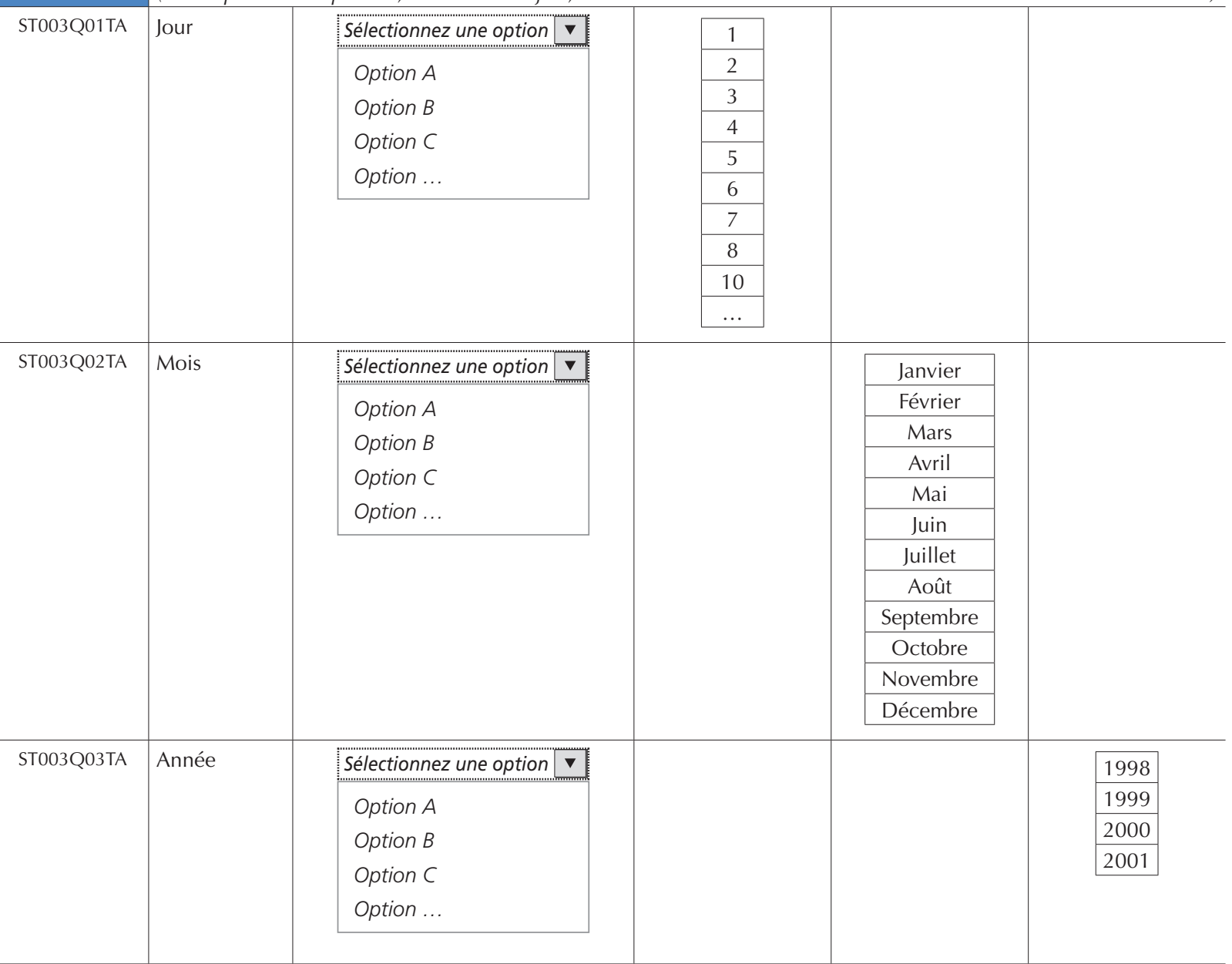

Contrôle de cohérence/rappel si le jour, le mois ou l'année de naissance est manquant : "Veuillez indiquer votre date complète de naissance ».

ST004

ST004Q01TA
Êtes-vous une fille ou un garçon?

(Sélectionnez une réponse.)
ST005
Quel est le <niveau de scolarité le plus élevé> que votre mère a terminé ?

Si vous hésitez sur la case à cocher, veuillez demander de l'aide à la <personne qui fait passer le test>.

(Sélectionnez une réponse.)

\begin{tabular}{|l|c|}
\hline$<$ Niveau CITE 3A> & $\square_{1}$ \\
\hline$<$ Niveau CITE 3B, 3C> & $\square_{2}$ \\
\hline$<$ Niveau CITE 2> & $\square_{3}$ \\
\hline$<$ Niveau CITE 1 > & $\square_{4}$ \\
\hline Elle n'a pas terminé le <niveau CITE 1 $>$ & $\square_{5}$ \\
\hline
\end{tabular}




\section{Votre mère a-t-elle obtenu un des diplômes suivants ?}

Si vous hésitez sur les réponses à cette question, veuillez demander de l'aide à la <personne qui fait passer le test>. (Sélectionnez une réponse par ligne.)

\begin{tabular}{l|l|c|c}
\multicolumn{2}{l|}{} & Oui & Non \\
\hline ST006Q01TA & $<$ Niveau CITE 6> & $\square_{1}$ & $\square_{2}$ \\
\hline ST006Q02TA & $<$ Niveau CITE 5A $>$ & $\square_{1}$ & $\square_{2}$ \\
\hline ST006Q03TA & <Niveau CITE 5B $>$ & $\square_{1}$ & $\square_{2}$ \\
\hline ST006Q04TA & <Niveau CITE 4> & $\square_{1}$ & $\square_{2}$ \\
\hline
\end{tabular}

ST007

ST007Q01TA
Quel est le <niveau de scolarité le plus élevé> que votre père a terminé ?

Si vous hésitez sur la case à cocher, veuillez demander de l'aide à <la personne qui fait passer le test>.

(Sélectionnez une réponse.)

$<$ Niveau CITE 3A>

$<$ Niveau CITE 3B, 3C $>$

$<$ Niveau CITE 2>

$<$ Niveau CITE 1>

Il n'a pas terminé le $<$ niveau CITE 1>

ST008

\section{Votre père a-t-il obtenu un des diplômes suivants ?}

Si vous hésitez sur les réponses à cette question, veuillez demander de l'aide à <la personne qui fait passer les tests>. (Sélectionnez une réponse par ligne.)

\begin{tabular}{l|l|c|c}
\multicolumn{2}{l|}{} & Oui & Non \\
\hline ST008Q01TA & $<$ Niveau CITE 6 $>$ & $\square_{1}$ & $\square_{2}$ \\
\hline ST008Q02TA & $<$ Niveau CITE 5A $>$ & $\square_{1}$ & $\square_{2}$ \\
\hline ST008Q03TA & $<$ Niveau CITE 5B $>$ & $\square_{1}$ & $\square_{2}$ \\
\hline ST008Q04TA & $<$ Niveau CITE 4 $>$ & $\square_{1}$ & $\square_{2}$ \\
\hline
\end{tabular}

ST011

À la maison, disposez-vous des choses suivantes ?

(Sélectionnez une réponse par ligne.)

\begin{tabular}{l|l|c|c} 
& Oui & Non \\
\hline ST011Q01TA & Un bureau ou une table pour travailler & $\square_{1}$ & $\square_{2}$ \\
\hline ST011Q02TA & Une chambre pour vous seul & $\square_{2}$ \\
\hline ST011Q03TA & Un endroit calme pour travailler & $\square_{1}$ & $\square_{2}$ \\
\hline ST011Q04TA & Un ordinateur dont vous pouvez vous servir pour votre travail scolaire & $\square_{2}$ \\
\hline ST011Q05TA & Des logiciels éducatifs & $\square_{1}$ & $\square_{2}$ \\
\hline ST011Q06TA & Une connexion à Internet & $\square_{1}$ & $\square_{2}$ \\
\hline ST011Q07TA & De la littérature classique (par exemple <Victor Hugo>) & $\square_{2}$ \\
\hline ST011Q08TA & Des recueils de poésie & $\square_{1}$ & $\square_{2}$ \\
\hline ST011Q09TA & Des œuuvres d'art (par exemple des tableaux) & $\square_{1}$ & $\square_{2}$ \\
\hline ST011Q10TA & Des livres utiles à votre travail scolaire & $\square_{1}$ & $\square_{1}$ \\
\hline ST011Q11TA & Des <ouvrages techniques de référence> & $\square_{2}$ \\
\hline ST011Q12TA & Un dictionnaire & $\square_{1}$ & $\square_{2}$ \\
\hline ST011Q16NA & Des livres sur l'art, la musique ou le design & $\square_{1}$ & $\square_{2}$ \\
\hline ST011Q17TA & <Item national d'aisance économique 1> & $\square_{1}$ & $\square_{2}$ \\
\hline ST011Q18TA & <Item national d'aisance économique 2> & $\square_{1}$ & $\square_{2}$ \\
\hline ST011Q19TA & <Item national d'aisance économique 3> & $\square_{1}$ & $\square_{2}$ \\
\hline
\end{tabular}


ST012

Y a-t-il chez vous les choses suivantes et, si oui, combien ?

(Sélectionnez une réponse par ligne.)

\begin{tabular}{|c|c|c|c|c|c|}
\hline & & $\operatorname{Aucun}(\mathrm{e})$ & Un(e) & Deux & Trois ou plus \\
\hline ST012Q01TA & Un poste de télévision & $\bigsqcup_{1}$ & $\square_{2}$ & $\square_{3}$ & $\square_{4}$ \\
\hline ST012Q02TA & Une voiture & & $\square_{2}$ & $\square_{3}$ & $\square_{4}$ \\
\hline ST012Q03TA & Une pièce équipée d'une baignoire ou d'une douche & $\square_{1}$ & $\square_{2}$ & $\square_{3}$ & \\
\hline ST012Q05NA & $\begin{array}{l}\text { Un <téléphone portable> avec connexion à Internet } \\
\text { (par ex. un smartphone) }\end{array}$ & & & & \\
\hline ST012Q06NA & $\begin{array}{l}\text { Un ordinateur (ordinateur de bureau, ordinateur portable } \\
\text { ou agenda électronique) }\end{array}$ & & & & \\
\hline ST012Q07NA & $\begin{array}{l}\text { Une }<\text { tablette tactile }>\text { (par ex. un }<\text { iPad }^{\oplus}> \\
\text { ou un }<\text { BlackBerry }^{\odot} \text { PlayBook }^{\mathrm{TM}}>\text { ) }\end{array}$ & $\square_{1}$ & $\square_{2}$ & & L \\
\hline ST012Q08NA & $\begin{array}{l}\text { Un }<\text { lecteur électronique }>\text { (par ex. }<\text { Kindle }^{\mathrm{TM}}>,<\text { Kobo }>\text {, } \\
<\text { Bookeen }>\text { ) }\end{array}$ & $\square_{1}$ & $\square_{2}$ & $\square$ & $\square_{4}$ \\
\hline ST012Q09NA & $\begin{array}{l}\text { Un instrument de musique (par ex. une guitare, } \\
\text { un piano) }\end{array}$ & $\square_{1}$ & $\square_{2}$ & $\square_{3}$ & $\square_{4}$ \\
\hline
\end{tabular}

ST013

ST013Q01TA

\section{Combien de livres y a-t-il chez vous?}

On compte environ 40 livres par mètre d'étagère. Ne tenez pas compte des magazines et des journaux, ni de vos manuels scolaires.

(Sélectionnez une réponse.)

\begin{tabular}{|l|c}
\hline $0-10$ livres & $\square_{1}$ \\
\hline $11-25$ livres & $\square_{2}$ \\
\hline 26-100 livres & $\square_{3}$ \\
\hline $101-200$ livres & $\square_{4}$ \\
\hline 201-500 livres & $\square_{5}$ \\
\hline Plus de 500 livres & $\square_{6}$ \\
\hline
\end{tabular}

ST014

ST014Q01TA

STO14Q02TA

ST014Q02TA
Les deux questions suivantes concernent l'activité professionnelle de votre mère :

(Si elle ne travaille pas actuellement, veuillez indiquer sa dernière activité professionnelle principale.)

Quelle est l'activité professionnelle principale de votre mère?

(Par ex. enseignante, aide-cuisinière, directrice des ventes.)

Tapez le nom de son travail :

Que fait votre mère dans le cadre de son activité professionnelle principale?

(Par ex. elle donne cours à des élèves de l'enseignement secondaire, elle aide le cuisinier d'un restaurant à préparer les repas, elle dirige une équipe de vente.)

En une phrase, indiquez en quoi consiste ou consistait son travail :

\footnotetext{
ST015Q01TA

ST015Q02TA

Les deux questions suivantes concernent l'activité professionnelle de votre père :

( $S^{\prime} i l$ ne travaille pas actuellement, veuillez indiquer sa dernière activité professionnelle principale.)

\begin{tabular}{c|l} 
ST015Q01TA & $\begin{array}{l}\text { Quelle est l'activité professionnelle principale de votre père? } \\
\text { (Par ex. enseignant, aide-cuisinier, directeur des ventes.) } \\
\text { Tapez le nom de son travail : }\end{array}$ \\
\hline ST015Q02TA & $\begin{array}{l}\text { Que fait votre père dans le cadre de son activité professionnelle principale? } \\
\text { (Par ex. il donne cours à des élèves dans l'enseignement secondaire, il aide le cuisinier d'un restaurant à préparer } \\
\text { les repas, il dirige une équipe de vente.) } \\
\text { En une phrase, indiquez en quoi consiste ou consistait son travail : }\end{array}$
\end{tabular}
}


Pensez à <cette année scolaire>. Dans quelle mesure êtes-vous d'accord ou non avec les affirmations suivantes?

(Sélectionnez une réponse par ligne.)

\begin{tabular}{c|l|c|c|c|c}
\multicolumn{2}{l|}{} & $\begin{array}{c}\text { Pas du } \\
\text { tout } \\
\text { d'accord }\end{array}$ & $\begin{array}{c}\text { Pas } \\
\text { d'accord }\end{array}$ & $\begin{array}{c}\text { D'accord } \\
\text { D'aut à fait } \\
\text { d'accord }\end{array}$ \\
\hline ST123Q01NA & Mes parents s'intéressent à mes activités scolaires. & $\square_{1}$ & $\square_{2}$ & $\square_{3}$ & $\square$ \\
\hline ST123Q02NA & Mes parents encouragent mes efforts et ma réussite scolaires. & $\square_{1}$ & $\square_{2}$ & $\square_{3}$ \\
\hline ST123Q03NA & $\begin{array}{l}\text { Mes parents me soutiennent quand je rencontre des difficultés } \\
\text { à l'école. }\end{array}$ & $\square_{1}$ & $\square_{2}$ & $\square_{3}$ \\
\hline ST123Q04NA & Mes parents m'encouragent à avoir confiance en moi. & $\square_{1}$ & $\square_{2}$ & $\square_{3}$ \\
\hline
\end{tabular}

\section{ST019}

Dans quel pays vos parents et vous-même êtes-vous nés?

(Sélectionnez une réponse par colonne.)

Vous $\quad$ Votre mère Votre père ST019Q01TA ST019Q01TB ST019Q01TC

\begin{tabular}{|l|c|c|c}
\hline <Pays A> & $\square_{1}$ & $\square_{1}$ & $\square_{1}$ \\
\hline$<$ Pays B> & $\square_{2}$ & $\square_{2}$ & $\square_{2}$ \\
\hline$<$ Pays C> & $\square_{3}$ & $\square_{3}$ & $\square_{3}$ \\
\hline$<$ Pays D> & $\square_{4}$ & $\square_{4}$ & $\square_{4}$ \\
\hline$<\ldots$ etc.> & $\square_{5}$ & $\square_{5}$ & $\square_{5}$ \\
\hline Autre pays & $\square_{6}$ & $\square_{6}$ & $\square_{6}$ \\
\hline
\end{tabular}

Il s'agit ici d'une question filtre. Si l'élève est né dans <le pays de l'évaluation>, passer la question ST021 et aller directement à la question ST022. Si l'élève N'EST PAS né dans <le pays de l'évaluation>, aller à la question ST021.

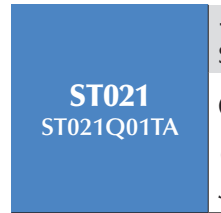

$\rightarrow$ Ne répondre que si l'option de réponse choisie dans la question ST019 pour la colonne « Vous » N'EST PAS « le pays de l'évaluation ». Sinon, passer à la question ST022.

Quel âge aviez-vous quand vous êtes arrivé(e) en <pays de l'évaluation> ?

(Sélectionnez votre réponse dans le menu déroulant.

Si vous aviez moins de 12 mois, veuillez sélectionner " entre 0 et 1 an » [entre zéro et un an].)

\begin{tabular}{|l|} 
Sélectionnez une option $\mathbf{\nabla}$ \\
\hline Option A \\
Option B \\
Option C \\
Option ...
\end{tabular}

\begin{tabular}{|c|c|}
\hline Entre 0 et 1 an & 1 \\
\hline 1 an & 2 \\
\hline 2 ans & 3 \\
\hline 3 ans & 4 \\
\hline 4 ans & 5 \\
\hline 5 ans & 6 \\
\hline 6 ans & 7 \\
\hline 7 ans & 8 \\
\hline 8 ans & 9 \\
\hline 9 ans & 10 \\
\hline 10 ans & 11 \\
\hline 11 ans & 12 \\
\hline 12 ans & 13 \\
\hline 13 ans & 14 \\
\hline 14 ans & 15 \\
\hline 15 ans & 16 \\
\hline 16 ans & 17 \\
\hline
\end{tabular}




\begin{tabular}{|c|c|c|}
\hline ST022 & $\begin{array}{l}\text { Quelle langue parlez-vous le plus souvent à la maison? } \\
\text { (Sélectionnez une réponse.) }\end{array}$ & \\
\hline ST022Q01TA & $<$ Langue $1>$ & $\square_{1}$ \\
\hline ST022Q02TA & $<$ Langue $2>$ & $\square_{2}$ \\
\hline ST022Q03TA & $<$ Langue $3>$ & $\square_{3}$ \\
\hline ST022Q04TA & $<\ldots$ etc. $>$ & $\square_{4}$ \\
\hline ST022Q05TA & Autre langue & $\square_{5}$ \\
\hline
\end{tabular}

ST125

ST125Q01NA
Quel âge aviez-vous lorsque vous êtes entré(e) au <niveau CITE $0>$ ?

(Pour répondre à la question, sélectionnez votre réponse dans le menu déroulant.)

\begin{tabular}{l|l|} 
Âge : & Sélectionnez une option $\mathbf{\nabla}$ \\
\hline & Option A \\
& Option B \\
& Option C \\
Option ....
\end{tabular}

Menu déroulant proposant les options de réponse suivantes : " 1 an ou moins », 2 ans, 3 ans, 4 ans, 5 ans, " 6 ans ou plus », " Je n'ai pas fréquenté le <niveau CITE $0>$ », et « Je ne m'en souviens pas ».

ST126

ST126Q01TA
Quel âge aviez-vous lorsque vous êtes entré au <niveau CITE 1> ?

(Pour répondre à la question, sélectionnez votre réponse dans le menu déroulant.)

Âge :

Sélectionnez une option

Option A

Option $B$

Option C

Option ...

Menu déroulant proposant les options de réponse suivantes : « 3 ans ou moins », 4 ans, 5 ans, 6 ans, 7 ans, 8 ans et « 9 ans ou plus ».

Vous est-il arrivé de redoubler un < grade> ?

(Sélectionnez une réponse par ligne.)

\begin{tabular}{|c|c|c|c|c|}
\hline & & Non, jamais & Oui, une fois & $\begin{array}{l}\text { Oui, deux } \\
\text { fois ou plus }\end{array}$ \\
\hline ST127Q01TA & Au $<$ niveau CITE $1>$ & $\square_{1}$ & $\square_{2}$ & $\square_{3}$ \\
\hline ST127Q02TA & Au $<$ niveau CITE 2> & $\square_{1}$ & $\square_{2}$ & $\square_{3}$ \\
\hline ST127Q03TA & Au $<$ niveau CITE $3>$ & $\square_{1}$ & $\square_{2}$ & $\square_{3}$ \\
\hline
\end{tabular}




\section{Ce que vous pensez de votre vie}

ST016

ST016Q01NA

La question suivante vous demande dans quelle mesure vous êtes satisfait(e) de votre vie sur une échelle de « 0 » à " 10 ». Zéro signifie que vous n'êtes " pas du tout satisfait(e) » et " 10 » signifie que vous êtes " entièrement satisfait(e)".

Dans l'ensemble, dans quelle mesure êtes-vous satisfait(e) de votre vie actuelle en général ?

(Positionnez le curseur sur le nombre qui vous convient.)

(Sélectionnez une réponse.)

\begin{tabular}{l|c}
$<$ Niveau CITE 2 $>$ & $\square_{1}$ \\
\hline$<$ Niveau CITE 3B ou C $>$ & $\square_{2}$ \\
\hline$<$ Niveau CITE 3A $>$ & $\square_{3}$ \\
\hline$<$ Niveau CITE 4 $>$ & $\square_{4}$ \\
\hline$<$ Niveau CITE 5B $>$ & $\square_{5}$ \\
\hline$<$ Niveau CITE 5A ou 6> & $\square_{6}$
\end{tabular}

ST114

Quel type de métier espérez-vous exercer quand vous aurez environ 30 ans ?

Tapez le nom de ce métier :

ST118

Dans quelle mesure êtes-vous d'accord ou non avec les affirmations suivantes à votre sujet ? (Sélectionnez une réponse par ligne.)

\begin{tabular}{|c|c|c|c|c|c|}
\hline & & $\begin{array}{l}\text { Pas du tout } \\
\text { d'accord }\end{array}$ & $\begin{array}{c}\text { Pas } \\
\text { d'accord }\end{array}$ & D'accord & $\begin{array}{l}\text { Tout à fait } \\
\text { d'accord }\end{array}$ \\
\hline ST118Q01NA & $\begin{array}{l}\text { J'ai souvent peur d'avoir des difficultés à réussir } \\
\text { un contrôle. }\end{array}$ & $\square_{1}$ & & $\square_{3}$ & $\square_{4}$ \\
\hline ST118Q02NA & J'ai peur d'avoir de mauvaises < notes> à l'école. & $\square_{1}$ & $\square_{2}$ & $\square_{3}$ & $\square_{4}$ \\
\hline ST118Q03NA & $\begin{array}{l}\text { Même si je me suis bien préparé(e) pour un contrôle, } \\
\text { je me sens très angoissé(e). }\end{array}$ & & & & $\square_{4}$ \\
\hline ST118Q04NA & Je suis très tendu(e) quand j'étudie pour un contrôle. & $\square_{1}$ & $\square_{2}$ & $\square_{3}$ & $\square_{4}$ \\
\hline ST118Q05NA & $\begin{array}{l}\text { Je deviens nerveux/nerveuse quand je ne sais pas } \\
\text { comment résoudre un exercice à l'école. }\end{array}$ & $\square_{1}$ & $\square_{2}$ & $\square_{3}$ & $\square_{4}$ \\
\hline
\end{tabular}

ST119

Dans quelle mesure êtes-vous d'accord ou non avec les affirmations suivantes à votre sujet ? (Sélectionnez une réponse par ligne.)

\begin{tabular}{c|l|c|c|c|c}
\multicolumn{2}{l|}{} & $\begin{array}{c}\text { Pas du tout } \\
\text { d'accord }\end{array}$ & $\begin{array}{c}\text { Pas } \\
\text { d'accord }\end{array}$ & $\begin{array}{c}\text { D'accord } \\
\text { D'aut à fait } \\
\text { d'accord }\end{array}$ \\
\hline ST119Q01NA & $\begin{array}{l}\text { Je veux avoir d'excellentes <notes> dans la plupart ou } \\
\text { dans tous mes cours. }\end{array}$ & $\square_{1}$ & $\square_{2}$ & $\square_{3}$ & $\square_{4}$ \\
\hline ST119Q02NA & $\begin{array}{l}\text { Je veux pouvoir choisir parmi les meilleures } \\
\text { opportunités possibles après avoir obtenu mon diplôme. }\end{array}$ & $\square_{1}$ & $\square_{2}$ & $\square_{3}$ & $\square_{4}$ \\
\hline ST119Q03NA & Je veux être le/la meilleur(e) dans tout ce que je fais. & $\square_{1}$ & $\square_{2}$ & $\square_{3}$ & $\square_{4}$ \\
\hline ST119Q04NA & Je me considère comme une personne ambitieuse. & $\square_{1}$ & $\square_{2}$ & $\square_{3}$ & $\square_{4}$ \\
\hline ST119Q05NA & Je veux être un(e) des meilleurs élèves de ma classe. & $\square_{1}$ & $\square_{2}$ & $\square_{3}$ & $\square_{4}$ \\
\hline
\end{tabular}


Lisez les trois descriptions d'élèves ci-après. En vous basant sur ces informations, dans quelle mesure êtes-vous d'accord ou non avec chaque conclusion affirmant que l'élève est motivé(e) ? (Sélectionnez une réponse par ligne.)

\begin{tabular}{l|l|c|c|c}
\multicolumn{1}{l|}{} & $\begin{array}{c}\text { Pas du tout } \\
\text { d'accord }\end{array}$ & $\begin{array}{c}\text { Pas } \\
\text { d'accord }\end{array}$ & $\begin{array}{c}\text { D'accord } \\
\text { DT121Q01NA }\end{array}$ & $\begin{array}{c}\text { Tout à fait } \\
\text { d'accord }\end{array}$ \\
\hline & $\begin{array}{l}\text { <PRÉNOM 1> abandonne facilement quand } \\
\text { il rencontre un problème et il ne se prépare pas souvent } \\
\text { pour les cours. } \\
\text { <Prénom 1> est motivé. }\end{array}$ & $\square_{1}$ & $\square_{3}$ \\
\hline ST121Q02NA & $\begin{array}{l}\text { Quand < PRÉNOM 2> commence un exercice, } \\
\text { souvent elle s'y intéresse jusqu'au bout et parfois, } \\
\text { elle en fait plus que demandé. } \\
\text { <Prénom 2> est motivée. }\end{array}$ & $\square_{1}$ & $\square_{2}$ & $\square_{3}$ \\
\hline ST121Q03NA & $\begin{array}{l}\text { <PRÉNOM 3> veut obtenir d'excellentes notes et il travaille } \\
\text { sur ses exercices jusqu'à ce que tout soit parfait. } \\
\text { <Prénom 3> est motivé. }\end{array}$ & $\square_{4}$ & $\square_{1}$ \\
\hline
\end{tabular}

\section{Votre établissement}

ST082

Dans quelle mesure êtes-vous d'accord ou non avec les affirmations suivantes à votre sujet ?

(Sélectionnez une réponse par ligne.)

\begin{tabular}{l|l|c|c|c|c}
\multicolumn{2}{l|}{} & $\begin{array}{c}\text { Pas du tout } \\
\text { d'accord }\end{array}$ & $\begin{array}{c}\text { Pas } \\
\text { d'accord }\end{array}$ & $\begin{array}{c}\text { Tout à fait } \\
\text { D'accord } \\
\text { d'accord }\end{array}$ \\
\hline ST082Q01NA & Je préfère travailler en groupe plutôt que seul(e). & $\square_{1}$ & $\square_{2}$ & $\square_{3}$ & $\square_{4}$ \\
\hline ST082Q02NA & J'ai le sens de l'écoute. & $\square_{1}$ & $\square_{2}$ & $\square_{3}$ & $\square_{4}$ \\
\hline ST082Q03NA & J'aime voir mes camarades de classe réussir. & $\square_{1}$ & $\square_{2}$ & $\square_{3}$ & $\square_{4}$ \\
\hline ST082Q08NA & Je tiens compte de ce qui intéresse les autres. & $\square_{1}$ & $\square_{2}$ & $\square_{3}$ & $\square_{4}$ \\
\hline ST082Q09NA & $\begin{array}{l}\text { Je trouve que les décisions prises en groupe sont } \\
\text { meilleures que celles prises individuellement. }\end{array}$ & $\square_{1}$ & $\square_{2}$ & $\square_{3}$ & $\square_{4}$ \\
\hline ST082Q12NA & $\begin{array}{l}\text { J'aime prendre en considération différentes } \\
\text { perspectives. }\end{array}$ & $\square_{1}$ & $\square_{2}$ & $\square_{3}$ & $\square$ \\
\hline ST082Q13NA & $\begin{array}{l}\text { Je trouve que le travail en groupe augmente } \\
\text { mes propres compétences. }\end{array}$ & $\square_{1}$ & $\square_{2}$ & $\square_{3}$ \\
\hline ST082Q14NA & J'aime travailler en collaboration avec des camarades. & $\square_{1}$ & $\square_{2}$ & $\square_{3}$ \\
\hline
\end{tabular}

Pensez à votre établissement. Dans quelle mesure êtes-vous d'accord ou non avec les affirmations suivantes?

(Sélectionnez une réponse par ligne.)

\begin{tabular}{c|l|c|c|c|c}
\multicolumn{2}{l|}{} & $\begin{array}{c}\text { Pas du tout } \\
\text { d'accord }\end{array}$ & $\begin{array}{c}\text { Pas } \\
\text { d'accord }\end{array}$ & $\begin{array}{c}\text { Tout à fait } \\
\text { D'accord } \\
\text { d'accord }\end{array}$ \\
\hline ST034Q01TA & Je me sens comme un étranger (ou hors du coup) à l'école. & $\square_{1}$ & $\square_{2}$ & $\square_{3}$ & $\square_{4}$ \\
\hline ST034Q02TA & Je me fais facilement des amis à l'école. & $\square_{1}$ & $\square_{2}$ & $\square_{3}$ & $\square_{4}$ \\
\hline ST034Q03TA & Je me sens chez moi à l'école. & $\square_{1}$ & $\square_{2}$ & $\square_{3}$ & $\square$ \\
\hline ST034Q04TA & Je me sens mal à l'aise, pas à ma place dans mon école. & $\square_{1}$ & $\square_{2}$ & $\square_{3}$ & $\square_{4}$ \\
\hline ST034Q05TA & Les autres élèves ont l'air de m'apprécier. & $\square_{1}$ & $\square_{2}$ & $\square_{3}$ & $\square$ \\
\hline ST034Q06TA & Je me sens seul à l'école. & $\square_{1}$ & $\square_{2}$ & $\square_{3}$ & $\square$ \\
\hline
\end{tabular}


ST039 Au cours des 12 derniers mois, à quelle fréquence avez-vous vécu les situations suivantes à l'école ? (Sélectionnez une réponse par ligne.)

\begin{tabular}{c|l|c|c|c|c}
\multicolumn{1}{c|}{} & $\begin{array}{c}\text { Jamais } \\
\text { ou presque } \\
\text { jamais }\end{array}$ & $\begin{array}{c}\text { Quelques } \\
\text { fois } \\
\text { par an }\end{array}$ & $\begin{array}{c}\text { Quelques } \\
\text { fois } \\
\text { par mois }\end{array}$ & $\begin{array}{c}\text { Au moins } \\
\text { une fois } \\
\text { par semaine }\end{array}$ \\
\hline ST039Q01NA & $\begin{array}{l}\text { Les professeurs ont moins souvent fait appel à moi } \\
\text { qu'aux autres élèves. }\end{array}$ & $\square_{1}$ & $\square_{2}$ & $\square_{3}$ & $\square_{4}$ \\
\hline ST039Q02NA & $\begin{array}{l}\text { Les professeurs m'ont noté(e) plus sévèrement } \\
\text { que les autres élèves. }\end{array}$ & $\square_{1}$ & $\square_{2}$ & $\square_{3}$ & $\square_{4}$ \\
\hline ST039Q03NA & $\begin{array}{l}\text { J'ai eu l'impression que les professeurs pensaient que } \\
\text { je suis moins intelligent(e) que je ne le suis vraiment. }\end{array}$ & $\square_{1}$ & $\square_{2}$ & $\square_{3}$ & $\square_{4}$ \\
\hline ST039Q04NA & $\begin{array}{l}\text { Les professeurs m'ont puni(e) plus durement que les autres } \\
\text { élèves. }\end{array}$ & $\square_{1}$ & $\square_{2}$ & $\square_{3}$ \\
\hline ST039Q05NA & Les professeurs m'ont ridiculisé(e) devant d'autres personnes. & $\square_{1}$ & $\square_{2}$ & $\square_{3}$ \\
\hline ST039Q06NA & $\begin{array}{l}\text { Les professeurs m'ont dit quelque chose d'insultant devant } \\
\text { d'autres personnes. }\end{array}$ & $\square_{1}$ & $\square_{2}$ & $\square_{3}$ \\
\hline
\end{tabular}

ST038

Au cours des 12 derniers mois, à quelle fréquence avez-vous vécu les situations suivantes à l'école ? (Sélectionnez une réponse par ligne.)

\begin{tabular}{|c|c|c|c|c|c|}
\hline & & $\begin{array}{c}\text { Jamais } \\
\text { ou presque } \\
\text { jamais }\end{array}$ & $\begin{array}{c}\text { Quelques } \\
\text { fois } \\
\text { par an }\end{array}$ & $\begin{array}{l}\text { Quelques } \\
\text { fois } \\
\text { par mois }\end{array}$ & $\begin{array}{c}\text { Au moins } \\
\text { une fois } \\
\text { par semaine }\end{array}$ \\
\hline ST038Q01NA & Des élèves m’ont insulté(e). & $\square_{1}$ & $\square_{2}$ & $\square_{3}$ & $\square_{4}$ \\
\hline ST038Q02NA & Des élèves s'en sont pris à moi. & $\square_{1}$ & $\square_{2}$ & $\square_{3}$ & $\square_{4}$ \\
\hline ST038Q03NA & Des élèves m’ont volontairement tenu(e) à l'écart. & $\square_{1}$ & $\square_{2}$ & $\square_{3}$ & $\square_{4}$ \\
\hline ST038Q04NA & Des élèves se sont moqués de moi. & $\square_{1}$ & $\square_{2}$ & $\square_{3}$ & $\square_{4}$ \\
\hline ST038Q05NA & Des élèves m’ont menacé(e) & $\square_{1}$ & $\square_{2}$ & $\square_{3}$ & $\square_{4}$ \\
\hline ST038Q06NA & $\begin{array}{l}\text { Des élèves se sont emparés ou ont détruit des objets } \\
\text { m'appartenant. }\end{array}$ & $\square_{1}$ & $\square_{2}$ & $\square_{3}$ & $\square_{4}$ \\
\hline ST038Q07NA & Des élèves m’ont frappé(e) ou bousculé(e). & $\square_{1}$ & $\square_{2}$ & $\square_{3}$ & $\square_{4}$ \\
\hline ST038Q08NA & Des élèves ont fait circuler de mauvaises rumeurs sur moi & $\square_{1}$ & $\square_{2}$ & $\square_{3}$ & $\square_{4}$ \\
\hline
\end{tabular}

\section{Votre horaire à l'école et le temps consacré à apprendre}

\begin{tabular}{c|l|l} 
ST059 & $\begin{array}{l}\text { Dans une semaine de cours ordinaire, combien de <périodes de cours }>\text { devez-vous suivre dans } \\
\text { les matières suivantes ? } \\
\text { (Veuillez indiquer le nombre de <périodes de cours> pour chacune des matières. Tapez « } 0 \text { » (zéro) si vous n'en avez pas.) }\end{array}$ \\
\hline ST059Q01TA & Nombre de <périodes de cours> de <langue de l'évaluation> par semaine & \\
\hline ST059Q02TA & Nombre de <périodes de cours> de mathématiques par semaine & \\
\hline ST059Q03TA & Nombre de <périodes de cours> de <sciences> par semaine & \\
\hline Ne répondre que par des nombres entiers. Contrôle de cohérence si l'une des réponses est supérieure à 15.
\end{tabular}

ST060

ST060Q01NA
Combien de <périodes de cours> devez-vous suivre en tout dans une semaine de cours ordinaire complète?

(Positionnez le curseur sur le nombre de <périodes de cours> par semaine qui convient.)

Nombre TOTAL de $<$ périodes de cours $>$ $\longleftarrow$

Curseur : position initiale, plage 0 -« 80 ou plus » ; graduation=1; contrôle de cohérence/rappel si la réponse est inférieure à 10 ou supérieure à 60 .

ST061

ST061Q01NA
En moyenne, combien de minutes dure une <période de cours > ?

(Positionnez le curseur sur le nombre de minutes par <périodes de cours> qui convient.) Nombre de minutes en moyenne dans une $<$ période de cours $>$ 
Au cours des deux dernières semaines de classe complètes, combien de fois les situations suivantes se sont-elles produites?

(Sélectionnez une réponse par ligne.)

\begin{tabular}{c|l|c|c|c}
\multicolumn{2}{l|}{} & $\begin{array}{c}\text { Une ou } \\
\text { Aucune fois }\end{array}$ & $\begin{array}{c}\text { Trois ou } \\
\text { quatre fois }\end{array}$ & $\begin{array}{c}\text { Cinq fois } \\
\text { ou plus }\end{array}$ \\
\hline ST062Q01TA & $\begin{array}{l}\text { J'ai <séché/manqué sans autorisation> toute une journée } \\
\text { de cours. }\end{array}$ & $\square_{1}$ \\
\hline ST062Q02TA & J'ai <séché/manqué sans autorisation> certains cours. & $\square_{1}$ \\
\hline ST062Q03TA & Je suis arrivé(e) en retard à l'école. & $\square_{3}$ \\
\hline
\end{tabular}

\begin{tabular}{c|l|l} 
& $\begin{array}{l}\text { Cette année scolaire, environ combien d'heures par semaine passez-vous à étudier les matières } \\
\text { suivantes en plus du temps prévu dans votre horaire de cours obligatoire ? } \\
\text { ST071 } \\
\text { (Comptez le nombre total d'heures que vous passez à faire vos devoirs, à suivre des cours supplémentaires et à étudier.) } \\
\text { (Positionnez le curseur sur le nombre total d'heures qui convient pour chaque matière. Si pour une matière vous } \\
\text { n'avez aucun devoir à faire, aucune leçon à étudier, ni ne suivez aucun cours supplémentaire, positionnez le curseur } \\
\text { sur " } 0 \text { » [zéro] pour cette matière.) }\end{array}$ \\
\hline ST071Q01NA & <Cours de sciences> & \\
\hline ST071Q02NA & Mathématiques & \\
\hline ST071Q03NA & <Langue de l'évaluation> & \\
\hline ST071Q04NA & <Langue étrangère> & \\
\hline ST071Q05NA & Autres & \\
\hline
\end{tabular}

Curseur : position initiale, plage 0 -« 30 heures par semaine ou plus » ; graduation=1 ; contrôle de cohérence/rappel si la réponse est supérieure à 20.

ST031

ST032

ST032Q01NA

Cette année scolaire-ci, combien de jours par semaine, en moyenne, assistez-vous au cours d'éducation physique?

(Sélectionnez votre réponse dans le menu déroulant.)

Sélectionnez une option $\mathbf{\nabla}$
Option A
Option B
Option C
Option ...

Menu déroulant, 0 -« < nombre de jours d'instruction par semaine calendrier $>$ ».

Au cours des 7 derniers jours, combien de jours avez-vous fait les activités physiques suivantes en dehors de l'école?

(Pour répondre à la question, sélectionnez une réponse dans chacun des menus déroulants.)

Des activités physiques modérées d'une durée totale d'au moins 60 minutes par jour (par ex. marcher, monter des escaliers, aller à l'école à vélo, une activité <spécifique au pays $>$ )

\begin{tabular}{l|l||l} 
& au pays $>$ ) & $\begin{array}{l}\text { Option A } \\
\text { Option B } \\
\text { Option C } \\
\text { Option ... }\end{array}$ \\
\hline ST032Q02NA & $\begin{array}{l}\text { Des activités physiques intenses d'une durée totale d'au moins 20 minutes par jour } \\
\text { qui vous ont fait beaucoup transpirer et vous ont essoufflé(e) (par ex. la course, } \\
\text { le cyclisme, l'aérobic, le football, le patinage, une activité <spécifique au pays }>\text { ) }\end{array}$ & $\begin{array}{l}\text { Sélectionnez une option } \mathbf{\nabla} \\
\text { Option } A \\
\text { Option B } \\
\text { Option C } \\
\text { Option .... }\end{array}$ \\
\hline
\end{tabular}

Menus déroulants proposant des options de réponse allant de 0 à 7 jours. 


\section{Les <cours de sciences> dans votre établissement}

ST063

Quels <cours de sciences> avez-vous suivis cette année scolaire ou l'année dernière ?

(Sélectionnez les réponses qui conviennent à chaque ligne.)

\begin{tabular}{|c|c|c|c|}
\hline & & Cette année & $\begin{array}{l}\text { L'année } \\
\text { dernière }\end{array}$ \\
\hline ST063Q01N & Physique & $\square_{1}$ & $\square_{1}$ \\
\hline ST063Q02N & Chimie & $\square_{1}$ & $\square_{1}$ \\
\hline ST063Q03N & Biologie & $\square_{1}$ & $\square_{1}$ \\
\hline ST063Q04N & $<$ Sciences de la Terre et de l'Univers $>$ & $\square_{1}$ & $\square_{1}$ \\
\hline ST063Q05N & Sciences appliquées et technologie (par ex. <exemple propre au pays >) & $\square_{1}$ & $\square_{1}$ \\
\hline ST063Q06N & $<$ Sciences générales ou intégrées $>$ (par ex. <exemple propre au pays $>$ ) & $\square$ & $\square_{1}$ \\
\hline
\end{tabular}

Il s'agit ici d'une question filtre. Les questions ST064 à ST107 ne sont applicables que si la somme des clics dans la catégorie « Cette année » est supérieure à 0 (l'élève a au moins suivi un cours de sciences cette année). Dans le cas contraire, passer les questions ST064 à ST107 et aller directement à la section « Votre avis sur les sciences».

\begin{tabular}{|c|c|c|c|c|}
\hline \multirow{3}{*}{ ST064 } & \multicolumn{4}{|c|}{$\begin{array}{l}\rightarrow \text { Cette question n'est applicable que si l'élève a répondu avoir suivi au moins un cours de sciences cette année scolaire } \\
\text { à la question ST063. }\end{array}$} \\
\hline & \multicolumn{4}{|c|}{$\begin{array}{l}\text { En ce qui concerne vos < cours de sciences>, pouvez-vous faire les choix suivants ? } \\
\text { (Sélectionnez une réponse par ligne.) }\end{array}$} \\
\hline & & $\begin{array}{c}\text { Non, pas } \\
\text { du tout }\end{array}$ & $\begin{array}{l}\text { Oui, dans } \\
\text { une certaine } \\
\text { mesure }\end{array}$ & $\begin{array}{c}\text { Oui, } \\
\text { je peux } \\
\text { choisir } \\
\text { librement }\end{array}$ \\
\hline ST064Q01NA & Je peux choisir le(s) <cours de sciences > que je veux étudier. & $\square_{1}$ & $\square_{2}$ & $\square_{3}$ \\
\hline ST064Q02NA & Je peux choisir le niveau de difficulté. & $\square_{1}$ & $\square_{2}$ & $\square_{3}$ \\
\hline ST064Q03NA & $\begin{array}{l}\text { Je peux choisir le nombre de }<\text { cours de sciences }>\text { ou de }<\text { périodes } \\
\text { de cours }>\text {. }\end{array}$ & $\square_{1}$ & $\square_{2}$ & $\square_{3}$ \\
\hline
\end{tabular}

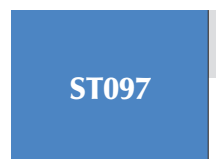

$\rightarrow$ Cette question n'est applicable que si l'élève a répondu avoir suivi au moins un cours de sciences cette année scolaire à la question ST063.

À quelle fréquence les situations suivantes se présentent-elles durant vos <cours de sciences $>$ ? (Sélectionnez une réponse par ligne.)

\begin{tabular}{c|l|c|c|c|c}
\multicolumn{2}{c|}{} & $\begin{array}{c}\text { À tous } \\
\text { les cours }\end{array}$ & $\begin{array}{c}\text { À la plupart } \\
\text { des cours }\end{array}$ & $\begin{array}{c}\text { À quelques } \\
\text { cours }\end{array}$ & $\begin{array}{c}\text { Jamais presque } \\
\text { jamais }\end{array}$ \\
\hline ST097Q01TA & Les élèves n'écoutent pas ce que dit le professeur. & $\square_{1}$ & $\square_{2}$ & $\square_{3}$ & $\square_{4}$ \\
\hline ST097Q02TA & Il y a du bruit et de l'agitation. & $\square_{1}$ & $\square_{2}$ & $\square_{3}$ & $\square$ \\
\hline ST097Q03TA & $\begin{array}{l}\text { Le professeur doit attendre un long moment avant } \\
\text { que les élèves se calment. }\end{array}$ & $\square_{1}$ & $\square_{2}$ & $\square_{3}$ & $\square_{4}$ \\
\hline ST097Q04TA & Les élèves ne peuvent pas bien travailler. & $\square_{1}$ & $\square_{2}$ & $\square_{3}$ & $\square_{4}$ \\
\hline ST097Q05TA & $\begin{array}{l}\text { Les élèves ne commencent à travailler que bien après } \\
\text { le début du cours. }\end{array}$ & $\square_{1}$ & $\square_{2}$ & $\square_{3}$ \\
\hline
\end{tabular}




\begin{tabular}{|c|c|c|c|c|c|}
\hline \multirow{3}{*}{ ST098 } & \multicolumn{5}{|c|}{$\begin{array}{l}\rightarrow \text { Cette question n'est applicable que si l'élève a répondu avoir suivi au moins un cours de sciences cette année scolaire } \\
\text { à la question ST063. }\end{array}$} \\
\hline & \multicolumn{5}{|c|}{$\begin{array}{l}\text { Lors de vos < cours de sciences>, à quelle fréquence les situations suivantes se produisent-elles? } \\
\text { (Sélectionnez une réponse par ligne.) }\end{array}$} \\
\hline & & $\begin{array}{l}\text { À chaque } \\
\text { cours }\end{array}$ & $\begin{array}{l}\text { À la plupart } \\
\text { des cours }\end{array}$ & $\begin{array}{l}\text { À quelques } \\
\text { cours }\end{array}$ & $\begin{array}{l}\text { Jamais } \\
\text { ou presque } \\
\text { jamais }\end{array}$ \\
\hline ST098Q01TA & Les élèves ont I'occasion d'exprimer leurs idées. & $\square_{1}$ & $\square_{2}$ & $\square_{3}$ & $\square_{4}$ \\
\hline ST098Q02TA & $\begin{array}{l}\text { Les élèves passent du temps au laboratoire pour } \\
\text { réaliser des expériences pratiques. }\end{array}$ & & & & \\
\hline ST098Q03NA & $\begin{array}{l}\text { Les élèves doivent construire des argumentations } \\
\text { sur des questions scientifiques. }\end{array}$ & & & & \\
\hline ST098Q05TA & $\begin{array}{l}\text { On demande aux élèves de tirer les conclusions } \\
\text { à partir des expériences qu'ils ont menées. }\end{array}$ & & 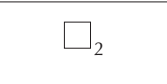 & & \\
\hline ST098Q06TA & $\begin{array}{l}\text { Le professeur explique comment un principe du <cours } \\
\text { de sciences }>\text { peut s'appliquer à divers phénomènes } \\
\text { (par ex. le mouvement des objets ou les substances } \\
\text { ayant des propriétés semblables). }\end{array}$ & & & & \\
\hline ST098Q07TA & $\begin{array}{l}\text { On permet aux élèves de concevoir leurs propres } \\
\text { expériences. }\end{array}$ & $\square$ & $\square_{2}$ & & \\
\hline ST098Q08NA & On débat en classe des projets de recherche. & $\square_{1}$ & $\square_{2}$ & $\square_{3}$ & $\square_{4}$ \\
\hline ST098Q09TA & $\begin{array}{l}\text { Le professeur explique clairement en quoi les concepts } \\
\text { de < scientifiques }>\text { sont importants dans notre vie. }\end{array}$ & $\square_{1}$ & $\square_{2}$ & & \\
\hline ST098Q10NA & $\begin{array}{l}\text { On demande aux élèves de mener une étude } \\
\text { scientifique pour tester leurs propres idées. }\end{array}$ & $\square_{1}$ & $\square_{2}$ & $\square_{3}$ & \\
\hline
\end{tabular}

En répondant aux questions suivantes, vous devrez penser à un seul de vos <cours de sciences> actuels. Vous pouvez choisir de quel cours il s'agit.

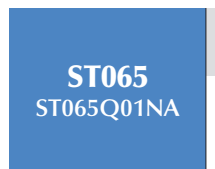

$\rightarrow$ Cette question n'est applicable que si l'élève a répondu avoir suivi au moins un cours de sciences cette année scolaire à la question ST063.

Quel est le nom de ce <cours de sciences>?

(Indiquez le nom du cours.)

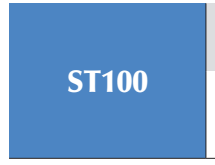

$\rightarrow$ Cette question n'est applicable que si l'élève a répondu avoir suivi au moins un cours de sciences cette année scolaire à la question ST063.

À quelle fréquence les situations suivantes se produisent-elles pendant vos <cours de sciences $>$ ?

(Sélectionnez une réponse par ligne.)

\begin{tabular}{c|l|c|c|c|c}
\multicolumn{1}{c|}{} & $\begin{array}{c}\text { À chaque } \\
\text { cours }\end{array}$ & $\begin{array}{c}\text { À la plupart } \\
\text { des cours }\end{array}$ & $\begin{array}{c}\text { À quelques } \\
\text { cours }\end{array}$ & $\begin{array}{c}\text { Jamais } \\
\text { ou presque } \\
\text { jamais }\end{array}$ \\
\hline ST100Q01TA & Le professeur s'intéresse aux progrès de chaque élève. & $\square_{1}$ & $\square_{2}$ & $\square_{3}$ & $\square_{4}$ \\
\hline ST100Q02TA & $\begin{array}{l}\text { Le professeur apporte de l'aide supplémentaire } \\
\text { quand les élèves en ont besoin. }\end{array}$ & $\square_{1}$ & $\square_{2}$ & $\square_{3}$ & $\square_{4}$ \\
\hline ST100Q03TA & Le professeur aide les élèves dans leur apprentissage. & $\square_{1}$ & $\square_{2}$ & $\square_{3}$ & $\square_{4}$ \\
\hline ST100Q04TA & $\begin{array}{l}\text { Le professeur continue à expliquer jusqu'à ce que } \\
\text { les élèves aient compris. }\end{array}$ & $\square_{1}$ & $\square_{2}$ & $\square_{3}$ & $\square_{4}$ \\
\hline ST100Q05TA & $\begin{array}{l}\text { Le professeur donne aux élèves l'occasion d'exprimer } \\
\text { leurs opinions. }\end{array}$ & $\square_{1}$ & $\square_{2}$ & $\square_{3}$ & $\square$ \\
\hline
\end{tabular}


$\rightarrow$ Cette question n'est applicable que si l'élève a répondu avoir suivi au moins un cours de sciences cette année scolaire à la question ST063.

À quelle fréquence les situations suivantes se produisent-elles pendant ce <cours de sciences> ?

(N'oubliez pas de répondre à cette question en pensant au <cours de sciences> que vous avez indiqué plus tôt.)

(Sélectionnez une réponse par ligne.)

\begin{tabular}{l|l|c|c|c|c}
\multicolumn{2}{l|}{} & $\begin{array}{c}\text { Jamais } \\
\text { ou presque } \\
\text { jamais }\end{array}$ & $\begin{array}{c}\text { À quelques } \\
\text { cours }\end{array}$ & $\begin{array}{c}\text { À la plupart } \\
\text { des cours }\end{array}$ & $\begin{array}{c}\text { À chaque } \\
\text { cours } \\
\text { ou presque }\end{array}$ \\
\hline ST103Q01NA & Le professeur explique des concepts scientifiques. & $\square_{1}$ & $\square_{2}$ & $\square_{3}$ & $\square_{4}$ \\
\hline ST103Q03NA & $\begin{array}{l}\text { Une discussion a lieu entre l'ensemble de la classe } \\
\text { et le professeur. }\end{array}$ & $\square_{1}$ & $\square_{2}$ & $\square_{3}$ & $\square$ \\
\hline ST103Q08NA & Le professeur discute de nos questions. & $\square_{1}$ & $\square_{2}$ & $\square_{3}$ & $\square_{4}$ \\
\hline ST103Q11NA & Le professeur démontre un concept. & $\square_{1}$ & $\square_{2}$ & $\square_{3}$ & $\square_{4}$ \\
\hline
\end{tabular}

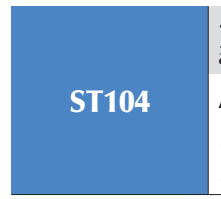

$\rightarrow$ Cette question n'est applicable que si l'élève a répondu avoir suivi au moins un cours de sciences cette année scolaire à la question ST063.

À quelle fréquence les situations suivantes se produisent-elles pendant ce <cours de sciences $>$ ?

(N’oubliez pas de répondre à cette question en pensant au <cours de sciences> que vous avez indiqué plus tôt.)

(Sélectionnez une réponse par ligne.)

\begin{tabular}{c|l|c|c|c|c}
\multicolumn{1}{c|}{} & $\begin{array}{c}\text { Jamais } \\
\text { ou presque } \\
\text { jamais }\end{array}$ & $\begin{array}{c}\text { À quelques } \\
\text { cours }\end{array}$ & $\begin{array}{c}\text { À la plupart } \\
\text { des cours }\end{array}$ & $\begin{array}{c}\text { À chaque } \\
\text { cours } \\
\text { ou presque }\end{array}$ \\
\hline ST104Q01NA & Le professeur me dit quels sont mes résultats à ce cours. & $\square_{1}$ & $\square_{2}$ & $\square_{3}$ & $\square$ \\
\hline ST104Q02NA & $\begin{array}{l}\text { Le professeur m'indique quels sont mes points forts } \\
\text { dans cette matière de <cours de sciences>. }\end{array}$ & $\square_{1}$ & $\square_{2}$ & $\square_{3}$ \\
\hline ST104Q03NA & $\begin{array}{l}\text { Le professeur me dit dans quels domaines je peux encore } \\
\text { m'améliorer. }\end{array}$ & $\square_{1}$ & $\square_{2}$ & $\square_{3}$ \\
\hline ST104Q04NA & $\begin{array}{l}\text { Le professeur me dit comment je peux améliorer } \\
\text { mes résultats. }\end{array}$ & $\square_{1}$ & $\square_{2}$ & $\square_{3}$ \\
\hline ST104Q05NA & $\begin{array}{l}\text { Le professeur me donne des conseils sur la façon } \\
\text { d'atteindre mes objectifs scolaires. }\end{array}$ & $\square_{1}$ & $\square_{2}$ & $\square_{4}$ \\
\hline
\end{tabular}

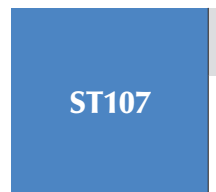

$\rightarrow$ Cette question n'est applicable que si l'élève a répondu avoir suivi au moins un cours de sciences cette année scolaire à la question ST063.

À quelle fréquence les situations suivantes se produisent-elles pendant ce <cours de sciences $>$ ?

(N’oubliez pas de répondre à cette question en pensant au <cours de sciences> que vous avez indiqué plus tôt.) (Sélectionnez une réponse par ligne.)

\begin{tabular}{c|l|c|c|c|c}
\multicolumn{2}{l|}{} & $\begin{array}{c}\text { Jamais } \\
\text { ou presque } \\
\text { jamais }\end{array}$ & $\begin{array}{c}\text { À quelques } \\
\text { cours }\end{array}$ & $\begin{array}{c}\text { À la plupart } \\
\text { des cours }\end{array}$ & $\begin{array}{c}\text { À chaque } \\
\text { cours presque } \\
\text { ou pres }\end{array}$ \\
\hline ST107Q01NA & $\begin{array}{l}\text { Le professeur adapte son cours aux besoins } \\
\text { et aux connaissances de la classe. }\end{array}$ & $\square_{1}$ & $\square_{2}$ & $\square_{3}$ \\
\hline ST107Q02NA & $\begin{array}{l}\text { Le professeur apporte une aide personnalisée quand } \\
\text { un élève a des difficultés à comprendre un sujet ou } \\
\text { un exercice. }\end{array}$ & $\square_{1}$ & $\square_{2}$ & $\square_{3}$ \\
\hline ST107Q03NA & $\begin{array}{l}\text { Le professeur modifie son cours quand la plupart } \\
\text { des élèves trouve le sujet difficile à comprendre. }\end{array}$ & $\square_{1}$ & $\square_{2}$ & $\square_{3}$ & $\square_{4}$ \\
\hline
\end{tabular}


Votre avis sur les sciences

ST092

Dans quelle mesure êtes-vous informé(e) sur les thèmes environnementaux suivants ?

(Sélectionnez une réponse par ligne.)

\begin{tabular}{|c|c|c|c|c|c|}
\hline & & $\begin{array}{c}\text { Je n'en } \\
\text { ai jamais } \\
\text { entendu } \\
\text { parler }\end{array}$ & $\begin{array}{c}\text { J'en ai } \\
\text { entendu } \\
\text { parler mais } \\
\text { je serais } \\
\text { incapable } \\
\text { d'expliquer } \\
\text { ce que c'est } \\
\text { exactement }\end{array}$ & $\begin{array}{c}\text { Je vois } \\
\text { de quoi il } \\
\text { s'agit et je } \\
\text { pourrais } \\
\text { expliquer } \\
\text { cela dans } \\
\text { les grandes } \\
\text { lignes }\end{array}$ & $\begin{array}{l}\text { Je connais } \\
\text { ce sujet et } \\
\text { je pourrais } \\
\text { clairement } \\
\text { expliquer de } \\
\text { quoi il s'agit }\end{array}$ \\
\hline ST092Q01TA & L'augmentation des gaz à effet de serre dans l'atmosphère & $\square_{1}$ & $\square_{2}$ & $\square_{3}$ & $\square_{4}$ \\
\hline ST092Q02TA & L'utilisation d'organismes génétiquement modifiés $(<\mathrm{OGM}>)$ & $\square_{1}$ & $\square_{2}$ & $\square_{3}$ & $\square_{4}$ \\
\hline ST092Q04TA & Les déchets nucléaires & $\square_{1}$ & $\square_{2}$ & $\square_{3}$ & $\square_{4}$ \\
\hline ST092Q05TA & $\begin{array}{l}\text { Les conséquences de l'abattage des forêts en vue } \\
\text { de l'exploitation des sols }\end{array}$ & $\square_{1}$ & $\square_{2}$ & $\square_{3}$ & $\square_{4}$ \\
\hline ST092Q06NA & La pollution de l'air & $\square_{1}$ & $\square_{2}$ & $\square_{3}$ & $\square_{4}$ \\
\hline ST092Q08NA & L'extinction de certaines espèces de plantes et d'animaux & $\square_{1}$ & $\square_{2}$ & $\square_{3}$ & $\square_{4}$ \\
\hline ST092Q09NA & Les pénuries d'eau & $\square_{1}$ & $\square_{2}$ & $\square_{3}$ & $\square_{4}$ \\
\hline
\end{tabular}

ST093

Pensez-vous que les risques liés aux problèmes environnementaux suivants s'atténueront ou s'aggraveront au cours des $\mathbf{2 0}$ prochaines années?

(Sélectionnez une réponse par ligne.)

\begin{tabular}{|c|c|c|c|c|}
\hline & & $\begin{array}{c}\text { Ils vont } \\
\text { s'atténuer }\end{array}$ & $\begin{array}{l}\text { Ils vont } \\
\text { rester à peu } \\
\text { près les } \\
\text { mêmes }\end{array}$ & $\begin{array}{c}\text { Ils vont } \\
\text { s'aggraver }\end{array}$ \\
\hline ST093Q01TA & La pollution de l'air & $\square_{1}$ & $\square_{2}$ & $\square_{3}$ \\
\hline ST093Q03TA & L'extinction de certaines plantes et animaux & $\square_{1}$ & $\square_{2}$ & $\square_{3}$ \\
\hline ST093Q04TA & L'abattage des forêts en vue de l'exploitation des sols & $\square_{1}$ & $\square_{2}$ & $\square_{3}$ \\
\hline ST093Q05TA & Les pénuries d'eau & $\square_{1}$ & $\square_{2}$ & $\square_{3}$ \\
\hline ST093Q06TA & Les déchets nucléaires & $\square_{1}$ & $\square_{2}$ & $\square_{3}$ \\
\hline ST093Q07NA & L'augmentation des gaz à effet de serre dans l'atmosphère & $\square_{1}$ & $\square_{2}$ & $\square_{3}$ \\
\hline ST093Q08NA & L'utilisation d'organismes génétiquement modifiés $(<\mathrm{OGM}>)$ & $\square_{1}$ & $\square_{2}$ & $\square_{3}$ \\
\hline
\end{tabular}

ST094

Dans quelle mesure êtes-vous d'accord ou non avec les affirmations suivantes à votre sujet ?

(Sélectionnez une réponse par ligne.)

\begin{tabular}{|c|c|c|c|c|c|}
\hline & & $\begin{array}{l}\text { Pas du tout } \\
\text { d'accord }\end{array}$ & $\begin{array}{c}\text { Pas } \\
\text { d'accord }\end{array}$ & D'accord & $\begin{array}{l}\text { Tout à fait } \\
\text { d'accord }\end{array}$ \\
\hline ST094Q01NA & $\begin{array}{l}\text { Je trouve généralement agréable d'apprendre des notions } \\
\text { de }<\text { sciences }>\text {. }\end{array}$ & $\square_{1}$ & $\square_{2}$ & $\square_{3}$ & $\square_{4}$ \\
\hline ST094Q02NA & J'aime lire des textes qui traitent de $<$ sciences $>$. & $\square_{1}$ & $\square_{2}$ & $\square_{3}$ & $\square_{4}$ \\
\hline ST094Q03NA & $\begin{array}{l}\text { Cela me plaît d'avoir à résoudre des problèmes } \\
\text { en }<\text { sciences }>\text {. }\end{array}$ & $\square_{1}$ & $\square_{2}$ & $\square_{3}$ & $\square_{4}$ \\
\hline ST094Q04NA & $\begin{array}{l}\text { Je prends plaisir à acquérir de nouvelles connaissances } \\
\text { en }<\text { sciences }>\text {. }\end{array}$ & $\square_{1}$ & $\square_{2}$ & $\square_{3}$ & $\square_{4}$ \\
\hline ST094Q05NA & $\begin{array}{l}\text { Cela m'intéresse d'apprendre des choses } \\
\text { sur les <sciences }>\text {. }\end{array}$ & $\square_{1}$ & $\square_{2}$ & $\square_{3}$ & $\square_{4}$ \\
\hline
\end{tabular}


ST095

Dans quelle mesure êtes-vous intéressé(e) par les sujets de <sciences> suivants ?

(Sélectionnez une réponse par ligne.)

\begin{tabular}{c|l|c|c|c|c|c}
\multicolumn{2}{l|}{} & $\begin{array}{c}\text { Pas } \\
\text { intéressé(e) }\end{array}$ & $\begin{array}{c}\text { Peu } \\
\text { intéressé(e) }\end{array}$ & $\begin{array}{c}\text { Je ne sais } \\
\text { pas ce que } \\
\text { c'est }\end{array}$ \\
\hline ST095Q04NA & $\begin{array}{l}\text { La biosphère (par ex. les écosystèmes, } \\
\text { le développement durable) }\end{array}$ & $\square_{1}$ & $\square_{2}$ & $\square_{3}$ & $\square_{4}$ \\
intéressé(e) & $\square_{5}$ \\
\hline ST095Q07NA & $\begin{array}{l}\text { Le mouvement et les forces } \\
\text { (par ex. la vitesse, la friction, les forces } \\
\text { magnétiques et gravitationnelles) }\end{array}$ & $\square_{1}$ & $\square_{2}$ & $\square_{3}$ & $\square_{4}$ & $\square$, \\
\hline ST095Q08NA & $\begin{array}{l}\text { L'énergie et sa transformation (par ex. } \\
\text { la conservation, les réactions chimiques) }\end{array}$ & $\square_{1}$ & $\square_{2}$ & $\square_{3}$ & $\square_{4}$ & $\square$ \\
\hline ST095Q13NA & L'Univers et son histoire & $\square_{1}$ & $\square_{2}$ & $\square_{3}$ & $\square_{4}$ & $\square$ \\
\hline ST095Q15NA & $\begin{array}{l}\text { La façon dont la science peut aider } \\
\text { à éviter certaines maladies }\end{array}$ & $\square_{1}$ & $\square_{2}$ & $\square_{3}$ & $\square_{4}$ & $\square$ \\
\hline
\end{tabular}

ST113

Dans quelle mesure êtes-vous d'accord ou non avec les affirmations ci-dessous ?

(Sélectionnez une réponse par ligne.)

\begin{tabular}{c|l|c|c|c|c}
\hline \multicolumn{2}{l|}{} & $\begin{array}{c}\text { Tout à fait } \\
\text { d'accord }\end{array}$ & D'accord & Pas d'accord & $\begin{array}{c}\text { Pas du tout } \\
\text { d'accord }\end{array}$ \\
\hline ST113Q01TA & $\begin{array}{l}\text { Cela vaut la peine de faire des efforts dans } \\
\text { le(s) <cours de sciences>, car cela m'aidera dans } \\
\text { le métier que je veux faire plus tard. }\end{array}$ & $\square_{1}$ & $\square_{2}$ & $\square_{3}$ \\
\hline ST113Q02TA & $\begin{array}{l}\text { Ce que j'apprends dans le(s) <cours de sciences> } \\
\text { est important pour moi, car j'en ai besoin pour ce que } \\
\text { je veux faire plus tard. }\end{array}$ & $\square_{1}$ & $\square_{2}$ & $\square_{3}$ \\
\hline ST113Q03TA & $\begin{array}{l}\text { Cela vaut la peine d'étudier pour le(s) } \\
\text { <cours de sciences>, car ce que j'apprends améliorera } \\
\text { mes perspectives de carrière professionnelle. }\end{array}$ & $\square_{1}$ & $\square_{2}$ & $\square_{3}$ & $\square_{4}$ \\
\hline ST113Q04TA & $\begin{array}{l}\text { Beaucoup de ce que j'apprends dans le(s) } \\
\text { <cours de sciences> m'aidera à trouver un emploi. }\end{array}$ & $\square_{1}$ & $\square_{2}$ & $\square_{3}$ & $\square_{4}$ \\
\hline
\end{tabular}

Dans quelle mesure vous serait-il facile d'effectuer seul(e) les tâches suivantes ?

(Sélectionnez une réponse par ligne.)

\begin{tabular}{|c|c|c|c|c|c|}
\hline & & $\begin{array}{l}\text { J'y arriverais } \\
\text { facilement }\end{array}$ & $\begin{array}{l}\text { J'y arriverais } \\
\text { avec un peu } \\
\text { d'effort }\end{array}$ & $\begin{array}{c}\text { Cela ne } \\
\text { me serait } \\
\text { pas facile } \\
\mathrm{d}^{\prime} \text { y arriver } \\
\text { seul(e) }\end{array}$ & $\begin{array}{l}\text { Je n'y } \\
\text { arriverais } \\
\text { pas }\end{array}$ \\
\hline ST129Q01TA & $\begin{array}{l}\text { Identifier la question scientifique qui est à la base } \\
\text { d'un article de journal portant sur un problème de santé. }\end{array}$ & $\square_{1}$ & $\square_{2}$ & $\square_{3}$ & $\square_{4}$ \\
\hline ST129Q02TA & $\begin{array}{l}\text { Expliquer pourquoi les tremblements de terre sont plus } \\
\text { fréquents dans certaines régions que dans d'autres. }\end{array}$ & $\square_{1}$ & $\square_{2}$ & $\square_{3}$ & $\square_{4}$ \\
\hline ST129Q03TA & $\begin{array}{l}\text { Décrire le rôle des antibiotiques dans le traitement } \\
\text { des maladies. }\end{array}$ & $\square_{1}$ & $\square_{2}$ & $\square_{3}$ & $\square_{4}$ \\
\hline ST129Q04TA & $\begin{array}{l}\text { Déterminer quelle est la question scientifique liée } \\
\text { au traitement des déchets. }\end{array}$ & $\square_{1}$ & $\square_{2}$ & $\square_{3}$ & $\square_{4}$ \\
\hline ST129Q05TA & $\begin{array}{l}\text { Prévoir en quoi des changements apportés } \\
\text { à l'environnement affecteront la survie de certaines } \\
\text { espèces. }\end{array}$ & $\square_{1}$ & $\square_{2}$ & $\square_{3}$ & $\square_{4}$ \\
\hline ST129Q06TA & $\begin{array}{l}\text { Interpréter des informations scientifiques fournies } \\
\text { sur l'étiquette des produits alimentaires. }\end{array}$ & $\square_{1}$ & $\square_{2}$ & $\square_{3}$ & $\square_{4}$ \\
\hline ST129Q07TA & $\begin{array}{l}\text { Discuter de la façon dont des données nouvelles } \\
\text { pourraient modifier votre point de vue sur la probabilité } \\
\text { qu'il existe de la vie sur Mars. }\end{array}$ & $\square_{1}$ & $\square_{2}$ & $\square_{3}$ & $\square_{4}$ \\
\hline ST129Q08TA & $\begin{array}{l}\text { Déterminer quelle est la meilleure de deux explications } \\
\text { sur la formation de pluies acides. }\end{array}$ & $\square_{1}$ & $\square_{2}$ & $\square_{3}$ & $\square_{4}$ \\
\hline
\end{tabular}


ST131

Dans quelle mesure êtes-vous d'accord ou non avec les affirmations suivantes ?

(Sélectionnez une réponse par ligne.)

\begin{tabular}{|c|c|c|c|c|c|}
\hline & & $\begin{array}{c}\text { Pas } \\
\text { du tout } \\
\text { d'accord }\end{array}$ & $\begin{array}{c}\text { Pas } \\
\text { d'accord }\end{array}$ & D'accord & $\begin{array}{l}\text { Tout à fait } \\
\text { d'accord }\end{array}$ \\
\hline ST131Q01NA & $\begin{array}{l}\text { Une bonne façon de savoir si quelque chose est vrai, } \\
\text { c'est de faire une expérience. }\end{array}$ & $\square_{1}$ & $\square_{2}$ & $\square_{3}$ & $\square_{4}$ \\
\hline ST131Q03NA & Il arrive que les concepts en <sciences > changent. & $\square_{1}$ & $\square_{2}$ & $\square_{3}$ & $\square_{4}$ \\
\hline ST131Q04NA & $\begin{array}{l}\text { Les bonnes réponses sont basées sur des éléments de preuve issus } \\
\text { de nombreuses expériences différentes. }\end{array}$ & $\square_{1}$ & $\square_{2}$ & $\square_{3}$ & $\square_{4}$ \\
\hline ST131Q06NA & $\begin{array}{l}\text { Il est préférable de répéter des expériences plusieurs fois pour } \\
\text { être sûr des résultats. }\end{array}$ & $\square_{1}$ & $\square_{2}$ & $\square_{3}$ & $\square_{4}$ \\
\hline ST131Q08NA & $\begin{array}{l}\text { Parfois les scientifiques changent d'avis sur ce qui est considéré } \\
\text { comme vrai en }<\text { sciences }>\text {. }\end{array}$ & $\square_{1}$ & $\square_{2}$ & $\square_{3}$ & $\square_{4}$ \\
\hline ST131Q11NA & $\begin{array}{l}\text { Il arrive que des concepts de }<\text { sciences }>\text { figurant dans des livres } \\
\text { de sciences changent. }\end{array}$ & $\square_{1}$ & $\square_{2}$ & $\square_{3}$ & $\square_{4}$ \\
\hline
\end{tabular}

ST146

À quelle fréquence vous arrive-t-il de faire ce qui suit ?

(Sélectionnez une réponse par ligne.)

\begin{tabular}{|c|c|c|c|c|c|}
\hline & & $\begin{array}{c}\text { Très } \\
\text { souvent }\end{array}$ & Régulièrement & Parfois & $\begin{array}{l}\text { Jamais } \\
\text { ou presque } \\
\text { jamais }\end{array}$ \\
\hline ST146Q01TA & $\begin{array}{l}\text { Regarder des programmes télévisés sur des thèmes } \\
\text { de < science au sens large }>\text {. }\end{array}$ & $\square_{1}$ & $\square_{2}$ & $\square_{3}$ & $\square_{4}$ \\
\hline ST146Q02TA & $\begin{array}{l}\text { Acheter ou emprunter des livres sur des thèmes } \\
\text { de < science au sens large }>\text {. }\end{array}$ & $\square_{1}$ & $\square_{2}$ & $\square_{3}$ & $\square_{4}$ \\
\hline ST146Q03TA & $\begin{array}{l}\text { Surfer sur des sites Web traitant de thèmes de < science } \\
\text { au sens large }>\text {. }\end{array}$ & $\square_{1}$ & $\square_{2}$ & $\square_{3}$ & $\square_{4}$ \\
\hline ST146Q04TA & $\begin{array}{l}\text { Lire des revues de }<\text { science au sens large }>\text { ou des articles } \\
\text { scientifiques dans les journaux. }\end{array}$ & $\square_{1}$ & $\square_{2}$ & $\square_{3}$ & $\square_{4}$ \\
\hline ST146Q05TA & Fréquenter un $<$ club de sciences $>$. & $\square_{1}$ & $\square_{2}$ & $\square_{3}$ & $\square_{4}$ \\
\hline ST146Q06NA & $\begin{array}{l}\text { Simuler des phénomènes naturels à l'aide de programmes } \\
\text { informatiques ou dans des laboratoires virtuels. }\end{array}$ & $\square_{1}$ & $\square_{2}$ & $\square_{3}$ & $\square_{4}$ \\
\hline ST146Q07NA & $\begin{array}{l}\text { Simuler des procédés techniques à l'aide de programmes } \\
\text { informatiques ou dans des laboratoires virtuels. }\end{array}$ & $\square_{1}$ & $\square_{2}$ & $\square_{3}$ & $\square_{4}$ \\
\hline ST146Q08NA & Visiter des sites Web d'organisations écologiques. & $\square_{1}$ & $\square_{2}$ & $\square_{3}$ & $\square_{4}$ \\
\hline ST146Q09NA & $\begin{array}{l}\text { Suivre les actualités des organisations scientifiques, } \\
\text { environnementales, écologiques sur des blogs } \\
\text { et des micro-blogs. }\end{array}$ & $\square_{1}$ & $\square_{2}$ & $\square_{3}$ & $\square_{4}$ \\
\hline
\end{tabular}


Le dernier jour où vous êtes allé(e) à l'école, avez-vous fait les choses suivantes le matin avant de partir?

(Sélectionnez une réponse par ligne.)

\begin{tabular}{|c|c|c|c|}
\hline & & Oui & Non \\
\hline ST076Q01NA & J'ai pris un petit déjeuner. & $\square_{1}$ & $\square_{2}$ \\
\hline ST076Q02NA & J'ai étudié pour l'école ou j'ai fait mes devoirs. & $\square_{1}$ & $\square_{2}$ \\
\hline ST076Q03NA & J'ai regardé la télévision/un <DVD>/une vidéo. & $\square_{1}$ & $\square_{2}$ \\
\hline ST076Q04NA & J'ai lu un livre/un journal/un magazine. & $\square_{1}$ & $\square_{2}$ \\
\hline ST076Q05NA & $\begin{array}{l}\text { Je suis allé(e) sur Internet/chatter/sur des réseaux sociaux (par ex. }<\text { Facebook }>\text {, } \\
<\text { réseau social propre au pays }>\text { ). }\end{array}$ & $\square_{1}$ & $\square_{2}$ \\
\hline ST076Q06NA & J'ai joué à des jeux vidéo. & $\square_{1}$ & $\square_{2}$ \\
\hline ST076Q07NA & J'ai rencontré des amis ou parlé avec des amis au téléphone. & $\square_{1}$ & $\square_{2}$ \\
\hline ST076Q08NA & J'ai parlé avec mes parents. & $\square_{1}$ & $\square_{2}$ \\
\hline ST076Q09NA & $\begin{array}{l}\text { J'ai fait des tâches ménagères ou je me suis occupé(e) d'autres membres } \\
\text { de la famille. }\end{array}$ & $\square_{1}$ & $\square_{2}$ \\
\hline ST076Q10NA & J'ai fait un travail pour lequel je suis payé(e). & $\square_{1}$ & $\square_{2}$ \\
\hline ST076Q11NA & J'ai fait de l'exercice ou du sport. & $\square_{1}$ & $\square_{2}$ \\
\hline
\end{tabular}

\section{ST078}

Le dernier jour où vous êtes allé(e) à l'école, avez-vous fait les choses suivantes après l'école ? (Sélectionnez une réponse par ligne.)

\begin{tabular}{|c|c|c|c|}
\hline & & Oui & Non \\
\hline ST078Q01NA & J'ai dîné. & $\square_{1}$ & $\square_{2}$ \\
\hline ST078Q02NA & J'ai étudié pour l'école ou j'ai fait mes devoirs. & $\square_{1}$ & $\square_{2}$ \\
\hline ST078Q03NA & J'ai regardé la télévision/un <DVD>/une vidéo. & $\square_{1}$ & $\square_{2}$ \\
\hline ST078Q04NA & J'ai lu un livre/un journal/un magazine. & $\square_{1}$ & $\square_{2}$ \\
\hline ST078Q05NA & $\begin{array}{l}\text { Je suis allé(e) sur Internet/chatter/sur des réseaux sociaux (par ex. <Facebook>, } \\
<\text { réseau social propre au pays }>\text { ). }\end{array}$ & $\square_{1}$ & $\square_{2}$ \\
\hline ST078Q06NA & J'ai joué à des jeux vidéo. & $\square_{1}$ & $\square_{2}$ \\
\hline ST078Q07NA & J'ai rencontré des amis ou parlé avec des amis au téléphone. & $\square_{1}$ & $\square_{2}$ \\
\hline ST078Q08NA & J'ai parlé avec mes parents. & $\square_{1}$ & $\square_{2}$ \\
\hline ST078Q09NA & $\begin{array}{l}\text { J'ai fait des tâches ménagères ou je me suis occupé(e) d'autres membres } \\
\text { de la famille. }\end{array}$ & $\square_{1}$ & $\square_{2}$ \\
\hline ST078Q10NA & J'ai fait un travail pour lequel je suis payé(e). & $\square_{1}$ & $\square_{2}$ \\
\hline ST078Q11NA & J'ai fait de l'exercice ou du sport. & $\square_{1}$ & $\square_{2}$ \\
\hline
\end{tabular}


QUESTIONNAIRE ÉLÈVE PORTANT SUR LE PARCOURS SCOLAIRE (Option internationale)

\section{Version source pour la campagne définitive}

Comme lors des enquêtes précédentes, d'autres questionnaires ont été élaborés. Ces questionnaires sont proposés à titre d'options aux pays et économies participants. Lors de l'enquête PISA 2015, ces questionnaires facultatifs incluent le questionnaire sur le parcours scolaire des élèves, le questionnaire sur leur maîtrise des TIC, le questionnaire «Parents» et le questionnaire « Enseignant(e)s».

Le questionnaire sur le parcours scolaire est destiné à recueillir des renseignements sur :

- Les cours supplémentaires suivis en sciences

- Les cours supplémentaires suivis en mathématiques

- Les cours supplémentaires suivis en <langue de l'évaluation>

- Le parcours scolaire

Les questions suivantes concernent les cours supplémentaires que vous suivez cette année dans vos matières scolaires ou autres. Ces cours peuvent avoir lieu à l'école ou ailleurs, mais ils ne font pas partie de votre programme de cours obligatoires. Veuillez prendre en compte toutes les activités supplémentaires institutionnalisées ou organisées que vous suivez régulièrement et où vous recevez une certaine forme d'enseignement, d'aide et de conseils ou de soutien (par ex. <exemples nationaux>).

\begin{tabular}{|c|c|c|}
\hline EC001 & \multicolumn{2}{|c|}{$\begin{array}{l}\text { Cette année scolaire-ci, combien d'heures par semaine environ suivez-vous des cours } \\
\text { supplémentaires en plus de vos cours scolaires obligatoires dans les domaines suivants? } \\
\text { (Une heure est égale à } 60 \text { minutes et non pas à la durée d'une période de cours.) } \\
\text { (Pour chaque domaine, positionnez le curseur sur le nombre d'heures que vous suivez, ou positionnez-le } \\
\text { sur " } O » \text { (zéro) si vous ne suivez pas de cours supplémentaires dans ce domaine) }\end{array}$} \\
\hline EC001Q01NA & Le $<$ cours de sciences $>$ ou les $<$ sciences au sens large $>$. & 0 \\
\hline EC001Q02NA & Mathématiques. & 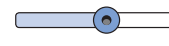 \\
\hline EC001Q03NA & $<$ Langue de l'évaluation>. & 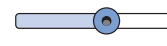 \\
\hline EC001Q04NA & <Langues étrangères > & $\lessdot \circ$ \\
\hline EC001Q05NA & Sciences sociales (par ex. I'histoire, la sociologie, la politique). & 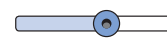 \\
\hline EC001Q06NA & $\begin{array}{l}\text { Musique (par ex. leçons pour apprendre à jouer d'un instrument de musique, } \\
\text { chorale, composition). }\end{array}$ & $(0$ \\
\hline EC001Q07NA & Sport (par ex. en clubs sportifs, en cours, en équipe). & 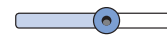 \\
\hline EC001Q08NA & Arts de la scène (par ex. la danse, le théâtre). & 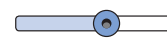 \\
\hline EC001Q09NA & Arts visuels (par ex. la photographie, le dessin, la sculpture). & 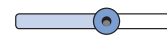 \\
\hline EC001Q10NA & Autres. & 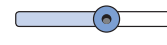 \\
\hline \multicolumn{3}{|c|}{$\begin{array}{l}\text { b) Les sections A, B et C présentées ci-après ne sont applicables que si l'élève suit des " cours supplémentaires » dans les domaines respectivement } \\
\text { concernés: la section A n'est applicable que si l'élève suit des " cours supplémentaires » en <cours de sciences> ou en <sciences au sens large>; } \\
\text { la section B, que si l'élève suit des " cours supplémentaires » en mathématiques; et la section C, que si l'élève suit des « cours supplémentaires " } \\
\text { en <langue de l'évaluation>. }\end{array}$} \\
\hline \multicolumn{3}{|c|}{$\begin{array}{l}\text { Tous les élèves ne suivant aucune forme de cours supplémentaires dans tout ou partie de ces domaines peuvent passer directement aux questions } \\
\text { leur demandant d'en indiquer les raisons. }\end{array}$} \\
\hline
\end{tabular}




\section{SECTION A COURS SUPPLÉMENTAIRES DE SCIENCES}

\begin{tabular}{|c|c|c|}
\hline \multirow{3}{*}{$\begin{array}{c}\text { EC003 } \\
\text { EC003Q01NA }\end{array}$} & \multirow{2}{*}{\multicolumn{2}{|c|}{$\begin{array}{l}\rightarrow \text { Les questions EC003 à EC012 ne sont applicables que si l'élève suit des « cours supplémentaires en sciences ». } \\
\text { Dans le cas contraire, passer les questions EC003 à EC012, et aller directement à la question EC013. } \\
\text { Quelles disciplines du <cours de sciences> figurent dans vos cours supplémentaires de sciences? } \\
\text { (Sélectionnez toutes les réponses qui conviennent.) }\end{array}$}} \\
\hline & & \\
\hline & Physique. & $\square_{1}$ \\
\hline EC003Q02NA & Chimie. & $\square_{1}$ \\
\hline EC003Q03NA & Biologie. & $\square_{1}$ \\
\hline EC003Q04NA & $<$ Sciences de la Terre et de I'Univers $>$. & $\square_{1}$ \\
\hline EC003Q05NA & Sciences appliquées et technologie (par ex. <exemple national>). & $\square_{1}$ \\
\hline EC003Q06NA & <Sciences générales ou intégrées> (par ex. <exemple national>). & $\square_{1}$ \\
\hline
\end{tabular}

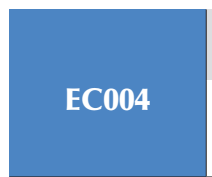

$\rightarrow$ Les questions EC003 à EC012 ne sont applicables que si l'élève suit des « cours supplémentaires en sciences ». Dans le cas contraire, passer les questions EC003 à EC012, et aller directement à la question EC013.

Quelle matière voyez-vous pendant ces cours supplémentaires de sciences?

(Sélectionnez une réponse par ligne.)

\begin{tabular}{l|l|c|c}
\hline \multicolumn{2}{l|}{ EC004Q01NA } & La matière vue pendant les cours ordinaires. & Oui \\
\hline EC004Q02NA & Une matière nouvelle ou complémentaire non vue pendant les cours ordinaires. & $\square_{1}$ & $\square_{2}$ \\
\hline
\end{tabular}

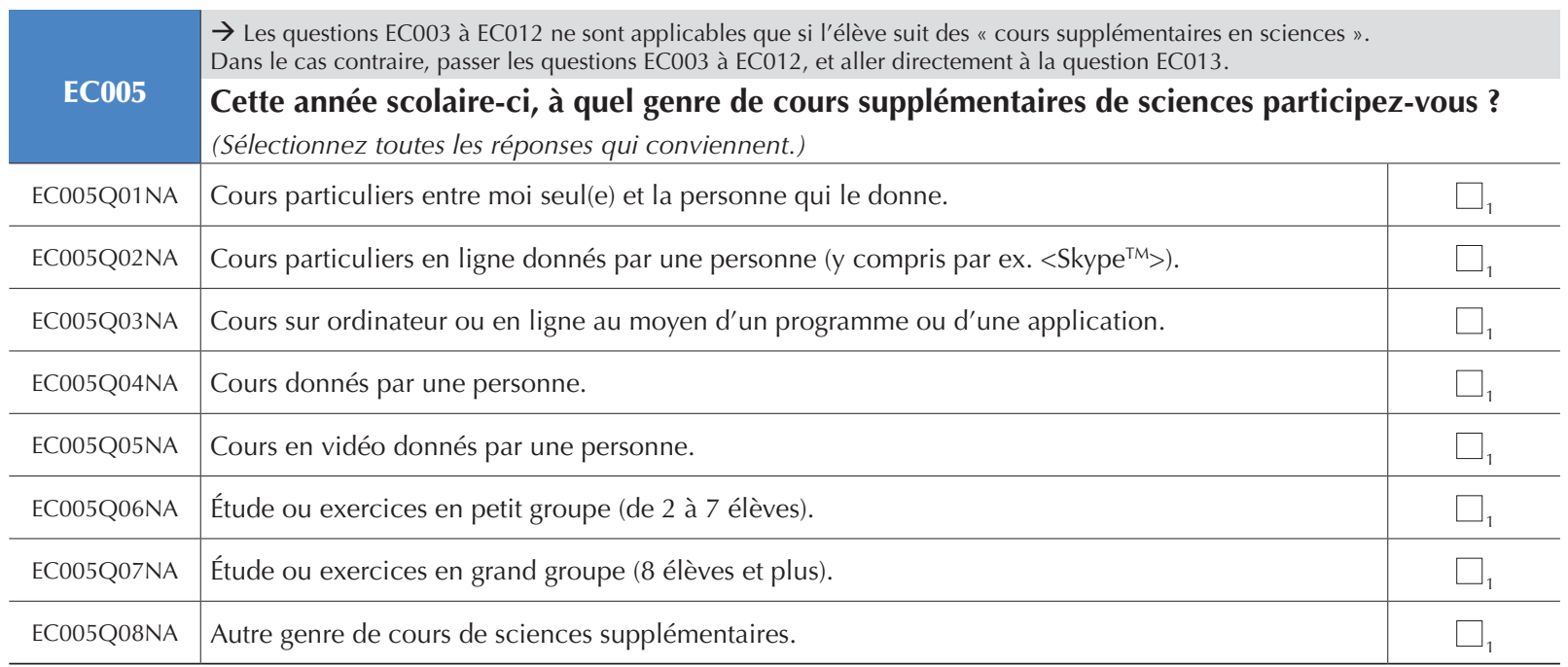

\begin{tabular}{c|l|l}
\multirow{2}{*}{ EC007 } & $\begin{array}{l}\rightarrow \text { Les questions EC003 à EC012 ne sont applicables que si l'élève suit des « cours supplémentaires en sciences ». } \\
\text { Dans le cas contraire, passer les questions EC003 à EC012, et aller directement à la question EC013. } \\
\text { Où suivez-vous ces cours supplémentaires de sciences ? } \\
\text { (Sélectionnez toutes les réponses qui conviennent.) }\end{array}$ \\
\hline EC007Q01NA & Dans mon établissement scolaire habituel. & $\square 1$ \\
\hline EC007Q02NA & Ailleurs, c'est-à-dire en dehors de mon établissement scolaire habituel. & $\square$, \\
\hline
\end{tabular}




\begin{tabular}{c|l|l|}
\multicolumn{1}{c|}{$\begin{array}{l}\rightarrow \text { Les questions EC003 à EC012 ne sont applicables que si l'élève suit des « cours supplémentaires en sciences ». } \\
\text { Dans le cas contraire, passer les questions EC003 à EC012, et aller directement à la question EC013. }\end{array}$} & $\begin{array}{l}\text { Parmi les descriptions suivantes, laquelle ou lesquelles correspond(ent) au professeur ou tuteur } \\
\text { qui vous donne des cours supplémentaires de sciences ? } \\
\text { (Sélectionnez toutes les réponses qui conviennent.) }\end{array}$ \\
\hline EC008Q01NA & Ce professeur est l'un des professeurs de mon école que j'ai cette année. \\
\hline EC008Q02NA & $\begin{array}{l}\text { Ce professeur enseigne régulièrement à des élèves de mon âge dans mon école mais ce n'est pas } \\
\text { un de mes professeurs. }\end{array}$ \\
\hline EC008Q03NA & $\begin{array}{l}\text { Ce professeur travaille principalement pour une société ou une organisation spécialisée } \\
\text { dans les cours supplémentaires. }\end{array}$ \\
\hline EC008Q04NA & Ce professeur n'est pas un enseignant avec une formation spécialisée (par ex. un étudiant). \\
\hline
\end{tabular}

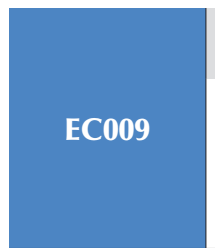

$\rightarrow$ Les questions EC003 à EC012 ne sont applicables que si l'élève suit des " cours supplémentaires en sciences ». Dans le cas contraire, passer les questions EC003 à EC012, et aller directement à la question EC013.

Comparez vos <cours de sciences> à l'école à vos cours supplémentaires de sciences. À quels cours est-il plus probable que le professeur agisse des façons suivantes ?

(Si vous avez plus d'un professeur de <cours de sciences> à l'école, choisissez-en un et un seul pour toutes les comparaisons.) (Sélectionnez une réponse par ligne.)

\begin{tabular}{l|l|c|c|c}
\multicolumn{2}{l|}{} & $\begin{array}{c}\text { Plutôt } \\
\text { à mes cours } \\
\text { ordinaires }\end{array}$ & $\begin{array}{c}\text { Pas de } \\
\text { différence }\end{array}$ & $\begin{array}{c}\text { Plutôt } \\
\text { à mes cours } \\
\text { supplémentaires }\end{array}$ \\
\hline EC009Q03NA & Mon professeur fait beaucoup pour m'aider. & $\square_{1}$ & $\square_{2}$ & $\square_{3}$ \\
\hline EC009Q07NA & $\begin{array}{l}\text { Mon professeur est content quand je trouve de nouvelles solutions } \\
\text { à un problème. }\end{array}$ & $\square_{1}$ & $\square_{2}$ & $\square_{3}$ \\
\hline EC009Q10NA & $\begin{array}{l}\text { Mon professeur donne des conseils ou propose des stratégies } \\
\text { qui m'aident à résoudre un exercice. }\end{array}$ & $\square_{1}$ & $\square_{2}$ & $\square_{3}$ \\
\hline EC009Q12NA & Mon professeur m'aide à trouver des façons de résoudre un problème. & $\square_{1}$ & $\square_{2}$ & $\square_{3}$ \\
\hline EC009Q13NA & $\begin{array}{l}\text { Une fois que nous savons pourquoi j'ai une difficulté particulière, } \\
\text { mon professeur me donne une stratégie de travail. }\end{array}$ & $\square_{1}$ & $\square_{2}$ & $\square_{3}$ \\
\hline EC009Q14NA & Mon professeur adapte le contenu et la méthode à mes besoins. & $\square_{1}$ & $\square_{2}$ & $\square_{3}$ \\
\hline
\end{tabular}

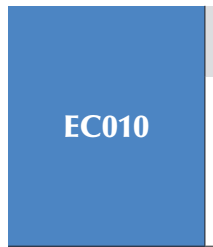

$\rightarrow$ Les questions EC003 à EC012 ne sont applicables que si l'élève suit des « cours supplémentaires en sciences ». Dans le cas contraire, passer les questions EC003 à EC012, et aller directement à la question EC013.

Comparez vos <cours de sciences> à l'école à vos cours supplémentaires de sciences. À quels cours est-il plus probable que les situations suivantes se produisent?

(Si vous avez plus d'un professeur de <cours de sciences> à l'école, choisissez-en un et un seul pour toutes les comparaisons.) (Sélectionnez une réponse par ligne.)

\begin{tabular}{l|l|c|c|c}
\multicolumn{1}{l|}{} & $\begin{array}{c}\text { Plutôt } \\
\text { à mes cours } \\
\text { ordinaires }\end{array}$ & $\begin{array}{c}\text { Pas de } \\
\text { différence }\end{array}$ & $\begin{array}{c}\text { Plutôt } \\
\text { à mes cours } \\
\text { supplémentaires }\end{array}$ \\
\hline EC010Q04NA & $\begin{array}{l}\text { II me faut beaucoup de temps pour rassembler tout le matériel } \\
\text { nécessaire pour commencer à travailler. }\end{array}$ & $\square_{1}$ & $\square_{2}$ & $\square_{3}$ \\
\hline EC010Q06NA & $\begin{array}{l}\text { Je parle de choses qui n'ont rien à voir avec les exercices à faire } \\
\text { ou avec le sujet du cours. }\end{array}$ & $\square_{1}$ & $\square_{2}$ & $\square_{3}$ \\
\hline EC010Q07NA & À la fin d'une leçon, mon professeur résume la matière qui a été vue. & $\square_{1}$ & $\square_{2}$ & $\square_{3}$ \\
\hline EC010Q08NA & Mon professeur souligne les aspects les plus importants du sujet. & $\square_{1}$ & $\square_{2}$ & $\square_{3}$ \\
\hline EC010Q09NA & Je m'ennuie souvent. & $\square_{1}$ & $\square_{2}$ & $\square_{3}$ \\
\hline EC010Q10NA & II me faut longtemps avant d'être prêt(e) à travailler. & $\square_{1}$ & $\square_{2}$ & $\square_{3}$ \\
\hline EC010Q11NA & $\begin{array}{l}\text { Mon professeur me dit ce que je vais apprendre dans une certaine } \\
\text { activité. }\end{array}$ & $\square_{1}$ & $\square_{2}$ & $\square_{3}$ \\
\hline EC010Q12NA & Mon professeur précise le contexte général de la leçon. & $\square_{1}$ & $\square_{2}$ & $\square_{3}$ \\
\hline
\end{tabular}


EC011

$\rightarrow$ Les questions EC003 à EC012 ne sont applicables que si l'élève suit des « cours supplémentaires en sciences ».

Dans le cas contraire, passer les questions EC003 à EC012, et aller directement à la question EC013.

Comparez vos < cours de sciences> à l'école à vos cours supplémentaires de sciences. À quels cours est-il plus probable que les rapports professeur-élève soient les suivants ?

(Si vous avez plus d'un professeur de <cours de sciences> à l'école, choisissez-en un et un seul pour toutes les comparaisons.) (Sélectionnez une réponse par ligne.)

\begin{tabular}{l|l|c|c|c}
\hline \multicolumn{2}{l|}{} & $\begin{array}{c}\text { Plutôt } \\
\text { à mes cours } \\
\text { ordinaires }\end{array}$ & $\begin{array}{c}\text { Pas de } \\
\text { différence }\end{array}$ & $\begin{array}{c}\text { Plutôt } \\
\text { à mes cours } \\
\text { supplémentaires }\end{array}$ \\
\hline EC011Q01NA & Je m'entends bien avec mon professeur. & $\square_{1}$ & $\square_{2}$ & $\square_{3}$ \\
\hline EC011Q02NA & Mon professeur s'intéresse à mon bien-être. & $\square_{1}$ & $\square_{2}$ & $\square_{3}$ \\
\hline EC011Q03NA & Mon professeur écoute vraiment ce que j'ai à dire. & $\square_{1}$ & $\square_{2}$ & $\square_{3}$ \\
\hline EC011Q04NA & Si j'ai besoin d'une aide supplémentaire, mon professeur me l'apporte. & $\square_{1}$ & $\square_{2}$ & $\square_{3}$ \\
\hline EC011Q05NA & Mon professeur est juste envers moi. & $\square_{1}$ & $\square_{2}$ & $\square_{3}$ \\
\hline
\end{tabular}

\begin{tabular}{|c|c|c|}
\hline \multirow[b]{2}{*}{ EC012 } & \multicolumn{2}{|l|}{$\begin{array}{l}\rightarrow \text { Les questions EC003 à EC012 ne sont applicables que si l'élève suit des " cours supplémentaires en sciences ». } \\
\text { Dans le cas contraire, passer les questions EC003 à EC012, et aller directement à la question EC013. }\end{array}$} \\
\hline & $\begin{array}{l}\text { Pourquoi suivez-vous des cours supplémentaires de sciences cette année scolaire-ci } \\
\text { (Sélectionnez toutes les réponses qui conviennent.) }\end{array}$ & \\
\hline EC012Q01NA & Je veux en apprendre davantage. & $\square_{1}$ \\
\hline EC012Q02NA & Je veux me préparer aux examens. & $\square_{1}$ \\
\hline EC012Q03NA & J'ai été attiré(e) par une publicité pour des cours de soutien. & $\square_{1}$ \\
\hline EC012Q04NA & Mes parents voulaient que je suive ces cours. & $\square_{1}$ \\
\hline EC012Q05NA & Beaucoup de mes amis le font. & $\square_{1}$ \\
\hline EC012Q06NA & Mes professeurs le recommandent. & $\square_{1}$ \\
\hline EC012Q07NA & Je veux améliorer mes notes. & $\square_{1}$ \\
\hline EC012Q08NA & Il faut que j'améliore mes notes. & $\square_{1}$ \\
\hline EC012Q09NA & Étudier m'apporte des satisfactions. & $\square_{1}$ \\
\hline EC012Q10NA & Cela fait bonne impression sur un curriculum vitae. & $\square_{1}$ \\
\hline EC012Q11NA & C'est nécessaire pour trouver un emploi. & $\square_{1}$ \\
\hline EC012Q12NA & Autre raison. & $\square_{1}$ \\
\hline
\end{tabular}

\begin{tabular}{l|l|c}
\multicolumn{1}{c|}{ EC013 } & $\begin{array}{l}\rightarrow \text { Uniquement si l'élève ne suit aucun « cours supplémentaires en sciences ». } \\
\text { Dans le cas contraire, passer cette question et aller directement à la question EC014. } \\
\text { Pourquoi ne suivez-vous pas de cours supplémentaires de sciences cette année scolaire-ci ? } \\
\text { (Sélectionnez toutes les réponses qui conviennent.) }\end{array}$ & $\square_{1}$ \\
\hline EC013Q01NA & Je n'ai pas besoin de cours supplémentaires de sciences. & $\square_{1}$ \\
\hline EC013Q02NA & Aucune des offres disponibles ne semblait convenir à mes besoins. & $\square_{1}$ \\
\hline EC013Q03NA & Il n'y a pas beaucoup de mes amis qui le font. & $\square_{1}$ \\
\hline EC013Q04NA & Je n'ai pas le temps. & $\square_{1}$ \\
\hline EC013Q05NA & Je n'ai pas de quoi les payer. & $\square_{1}$ \\
\hline EC013Q06NA & Mes professeurs de l'école s'y connaissent suffisamment. & $\square_{1}$ \\
\hline EC013Q07NA & Mes parents ne veulent pas. & $\square_{1}$ \\
\hline EC013Q08NA & Ils semblent trop chers pour ce qu'ils sont. & $\square_{1}$ \\
\hline EC013Q09NA & Mes professeurs disent que ce n'est pas utile. & $\square_{1}$ \\
\hline EC013Q10NA & Je n'ai jamais envisagé de prendre des cours supplémentaires de sciences. & $\square_{1}$ \\
\hline EC013Q11NA & Il n'y a pas de cours supplémentaires de sciences disponibles là où j'habite. & $\square_{1}$ \\
\hline EC013Q12NA & C'est ma famille qui m'aide. & $\square_{1}$ \\
\hline EC013Q13NA & Ce sont mes amis et mes camarades de classe qui m'aident. &
\end{tabular}




\section{SECTION B COURS SUPPLÉMENTAIRES DE MATHÉMATIQUES}

\begin{tabular}{|c|c|c|c|}
\hline \multirow{3}{*}{ EC014 } & \multicolumn{3}{|c|}{$\begin{array}{l}\rightarrow \text { Les questions EC014 à EC022 ne sont applicables que si l'élève suit des « cours supplémentaires en mathématiques ». } \\
\text { Dans le cas contraire, passer les questions EC014 à EC022, et aller directement à la question EC023. }\end{array}$} \\
\hline & \multicolumn{3}{|c|}{$\begin{array}{l}\text { Quelle matière voyez-vous pendant ces cours supplémentaires de mathématiques? } \\
\text { (Sélectionnez une réponse par ligne.) }\end{array}$} \\
\hline & & Oui & Non \\
\hline EC014Q01NA & La matière vue pendant les cours ordinaires. & $\square_{1}$ & $\square_{2}$ \\
\hline EC014Q02NA & Une matière nouvelle ou complémentaire non vue pendant les cours ordinaires. & $\square_{1}$ & $\square_{2}$ \\
\hline
\end{tabular}

\begin{tabular}{|c|c|c|}
\hline \multirow[b]{2}{*}{ EC015 } & \multicolumn{2}{|c|}{$\begin{array}{l}\rightarrow \text { Les questions EC014 à EC022 ne sont applicables que si l'élève suit des « cours supplémentaires en mathématiques ». } \\
\text { Dans le cas contraire, passer les questions EC014 à EC022, et aller directement à la question EC023. }\end{array}$} \\
\hline & \multicolumn{2}{|c|}{$\begin{array}{l}\text { Cette année scolaire-ci, à quel genre de cours supplémentaires de mathématiques participez-vous? } \\
\text { (Sélectionnez toutes les réponses qui conviennent.) }\end{array}$} \\
\hline EC015Q01NA & Cours particuliers entre moi seul(e) et la personne qui le donne. & $\square_{1}$ \\
\hline EC015Q02NA & Cours particuliers en ligne donnés par une personne (y compris par ex. $<\mathrm{Skype}^{\mathrm{TM}}>$ ). & $\square_{1}$ \\
\hline EC015Q03NA & Cours sur ordinateur ou en ligne au moyen d'un programme ou d'une application. & $\square_{1}$ \\
\hline EC015Q04NA & Cours donnés par une personne. & $\square_{1}$ \\
\hline EC015Q05NA & Cours en vidéo donnés par une personne. & $\square_{1}$ \\
\hline EC015Q06NA & Étude ou exercices en petit groupe (de 2 à 7 élèves). & $\square_{1}$ \\
\hline EC015Q07NA & Étude ou exercices en grand groupe (8 élèves et plus). & $\square_{1}$ \\
\hline EC015Q08NA & Autre genre de cours de mathématiques supplémentaire. & $\square_{1}$ \\
\hline
\end{tabular}

\begin{tabular}{c|l|c}
\multirow{2}{*}{ EC017 } & $\begin{array}{l}\rightarrow \text { Les questions EC014 à EC022 ne sont applicables que si l'élève suit des « cours supplémentaires en mathématiques ». } \\
\text { Dans le cas contraire, passer les questions EC014 à EC022, et aller directement à la question EC023. } \\
\text { Où suivez-vous ces cours supplémentaires de mathématiques ? } \\
\text { (Sélectionnez toutes les réponses qui conviennent.) }\end{array}$ \\
\hline EC017Q01NA & Dans mon établissement scolaire habituel. & $\square$, \\
\hline EC017Q02NA & Ailleurs, c'est-à-dire en dehors de mon établissement scolaire habituel. & $\square$, \\
\hline
\end{tabular}

\begin{tabular}{c|l|c}
\multicolumn{1}{c|}{$\begin{array}{l}\text { EC018 Les questions EC014 à EC022 ne sont applicables que si l'élève suit des « cours supplémentaires en mathématiques ». } \\
\text { Dans le cas contraire, passer les questions EC014 à EC022, et aller directement à la question EC023. }\end{array}$} & $\begin{array}{l}\text { Parmi les descriptions suivantes, laquelle ou lesquelles correspond(ent) au professeur ou tuteur } \\
\text { qui vous donne des cours supplémentaires de mathématiques ? } \\
\text { (Sélectionnez toutes les réponses qui conviennent.) }\end{array}$ \\
\hline EC018Q01NA & Ce professeur est l'un des professeurs de mon école que j'ai cette année. & $\square$ \\
\hline EC018Q02NA & $\begin{array}{l}\text { Ce professeur enseigne régulièrement à des élèves de mon âge dans mon école mais ce n'est pas } \\
\text { un de mes professeurs. }\end{array}$ \\
\hline EC018Q03NA & $\begin{array}{l}\text { Ce professeur travaille principalement pour une société ou une organisation spécialisée } \\
\text { dans les cours supplémentaires. }\end{array}$ \\
\hline EC018Q04NA & Ce professeur n'est pas un enseignant qualifié (par ex. un étudiant). \\
\hline
\end{tabular}




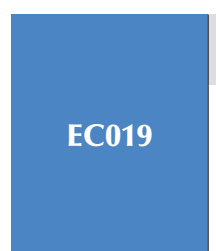

$\rightarrow$ Les questions EC014 à EC022 ne sont applicables que si l'élève suit des " cours supplémentaires en mathématiques ». Dans le cas contraire, passer les questions EC014 à EC022, et aller directement à la question EC023.

Comparez vos cours de mathématiques à l'école à vos cours supplémentaires de mathématiques. À quels cours est-il plus probable que le professeur agisse des façons suivantes?

(Si vous avez plus d'un professeur de mathématiques à l'école, choisissez-en un et un seul pour toutes les comparaisons.) (Sélectionnez une réponse par ligne.)

\begin{tabular}{|c|c|c|c|c|}
\hline & & $\begin{array}{l}\text { Plutôt } \\
\text { à mes cours } \\
\text { ordinaires }\end{array}$ & $\begin{array}{l}\text { Pas de } \\
\text { différence }\end{array}$ & $\begin{array}{c}\text { Plutôt } \\
\text { à mes cours } \\
\text { supplémentaires }\end{array}$ \\
\hline EC019Q03NA & Mon professeur fait beaucoup pour m'aider. & $\square_{1}$ & $\square_{2}$ & $\square_{3}$ \\
\hline EC019Q07NA & $\begin{array}{l}\text { Mon professeur est content quand je trouve de nouvelles solutions } \\
\text { à un problème. }\end{array}$ & & & \\
\hline EC019Q10NA & $\begin{array}{l}\text { Mon professeur donne des conseils ou propose des stratégies } \\
\text { qui m’aident à résoudre un exercice. }\end{array}$ & & & \\
\hline EC019Q12NA & Mon professeur m'aide à trouver des façons de résoudre un problème. & $\square_{1}$ & $\square_{2}$ & $\square_{3}$ \\
\hline EC019Q13NA & $\begin{array}{l}\text { Une fois que nous savons pourquoi j'ai une difficulté particulière, } \\
\text { mon professeur me donne une stratégie de travail. }\end{array}$ & $\square_{1}$ & & $\square_{3}$ \\
\hline EC019Q14NA & Mon professeur adapte le contenu et la méthode à mes besoins. & $\square_{1}$ & $\square_{2}$ & $\square_{3}$ \\
\hline
\end{tabular}

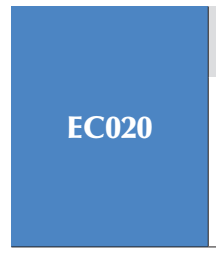

$\rightarrow$ Les questions EC014 à EC022 ne sont applicables que si l'élève suit des « cours supplémentaires en mathématiques ». Dans le cas contraire, passer les questions EC014 à EC022, et aller directement à la question EC023.

Comparez vos cours de mathématiques à l'école à vos cours supplémentaires de mathématiques. À quels cours est-il plus probable que les situations suivantes se produisent ?

(Si vous avez plus d'un professeur de mathématiques à l'école, choisissez-en un et un seul pour toutes les comparaisons.) (Sélectionnez une réponse par ligne.)

\begin{tabular}{|c|c|c|c|c|}
\hline & & $\begin{array}{c}\text { Plutôt } \\
\text { à mes cours } \\
\text { ordinaires }\end{array}$ & $\begin{array}{l}\text { Pas de } \\
\text { différence }\end{array}$ & $\begin{array}{c}\text { Plutôt } \\
\text { à mes cours } \\
\text { supplémentaires }\end{array}$ \\
\hline EC020Q04NA & $\begin{array}{l}\text { Il me faut beaucoup de temps pour rassembler tout le matériel nécessaire } \\
\text { pour commencer à travailler. }\end{array}$ & $\square_{1}$ & $\square_{2}$ & $\square_{3}$ \\
\hline EC020Q06NA & $\begin{array}{l}\text { Je parle de choses qui n'ont rien à voir avec les exercices à faire } \\
\text { ou avec le sujet du cours. }\end{array}$ & $\square_{1}$ & $\square_{2}$ & $\square_{3}$ \\
\hline EC020Q07NA & À la fin d'une leçon, mon professeur résume la matière qui a été vue. & $\square_{1}$ & $\square_{2}$ & $\square_{3}$ \\
\hline EC020Q08NA & Mon professeur souligne les aspects les plus importants du sujet. & $\square_{1}$ & $\square_{2}$ & $\square_{3}$ \\
\hline EC020Q09NA & Je m'ennuie souvent. & $\square_{1}$ & $\square_{2}$ & $\square_{3}$ \\
\hline EC020Q10NA & Il me faut longtemps avant d'être prêt(e) à travailler. & $\square_{1}$ & $\square_{2}$ & $\square_{3}$ \\
\hline EC020Q11NA & $\begin{array}{l}\text { Mon professeur me dit ce que je vais apprendre dans une certaine } \\
\text { activité. }\end{array}$ & $\square_{1}$ & $\square_{2}$ & $\square_{3}$ \\
\hline EC020Q12NA & Mon professeur précise le contexte général de la leçon. & $\square_{1}$ & $\square_{2}$ & $\square_{3}$ \\
\hline
\end{tabular}




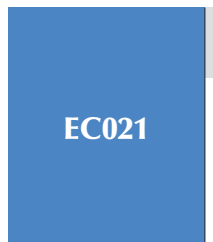

$\rightarrow$ Les questions EC014 à EC022 ne sont applicables que si l'élève suit des « cours supplémentaires en mathématiques ». Dans le cas contraire, passer les questions EC014 à EC022, et aller directement à la question EC023.

Comparez vos cours de mathématiques à l'école à vos cours supplémentaires de mathématiques. À quels cours est-il plus probable que les rapports professeur-élève soient les suivants ?

(Si vous avez plus d'un professeur de mathématiques à l'école, choisissez-en un et un seul pour toutes les comparaisons.) (Sélectionnez une réponse par ligne.)

\begin{tabular}{l|l|c|c|c}
\multicolumn{2}{|l|}{} & $\begin{array}{c}\text { Plutôt } \\
\text { à mes cours } \\
\text { ordinaires }\end{array}$ & $\begin{array}{c}\text { Pas de } \\
\text { différence }\end{array}$ & $\begin{array}{c}\text { Plutôt } \\
\text { à mes cours } \\
\text { supplémentaires }\end{array}$ \\
\hline EC021Q01NA & Je m'entends bien avec mon professeur. & $\square_{1}$ & $\square_{2}$ & $\square_{3}$ \\
\hline EC021Q02NA & Mon professeur s'intéresse à mon bien-être. & $\square_{1}$ & $\square_{2}$ & $\square_{3}$ \\
\hline EC021Q03NA & Mon professeur écoute vraiment ce que j'ai à dire. & $\square_{1}$ & $\square_{2}$ & $\square_{3}$ \\
\hline EC021Q04NA & Si j'ai besoin d'une aide supplémentaire, mon professeur me l'apporte. & $\square_{1}$ & $\square_{2}$ & $\square_{3}$ \\
\hline EC021Q05NA & Mon professeur est juste envers moi. & $\square_{1}$ & $\square_{2}$ & $\square_{3}$ \\
\hline
\end{tabular}

\begin{tabular}{l|l|c}
\multicolumn{1}{c|}{$\begin{array}{l}\text { EC022 Les questions EC014 à EC022 ne sont applicables que si l'élève suit des « cours supplémentaires en mathématiques ». } \\
\text { Dans le cas contraire, passer les questions EC014 à EC022, et aller directement à la question EC023. }\end{array}$} & $\begin{array}{l}\text { Pourquoi suivez-vous des cours supplémentaires de mathématiques cette année scolaire-ci ? } \\
\text { (Sélectionnez toutes les réponses qui conviennent.) }\end{array}$ \\
\hline EC022Q01NA & Je veux en apprendre davantage. & $\square_{1}$ \\
\hline EC022Q02NA & Je veux me préparer aux examens. & $\square_{1}$ \\
\hline EC022Q03NA & J'ai été attiré(e) par une publicité pour des cours de soutien. & $\square_{1}$ \\
\hline EC022Q04NA & Mes parents voulaient que je suive ces cours. & $\square_{1}$ \\
\hline EC022Q05NA & Beaucoup de mes amis le font. & $\square_{1}$ \\
\hline EC022Q06NA & Mes professeurs le recommandent. & $\square_{1}$ \\
\hline EC022Q07NA & Je veux améliorer mes notes. & $\square_{1}$ \\
\hline EC022Q08NA & Il faut que j'améliore mes notes. & $\square_{1}$ \\
\hline EC022Q09NA & Étudier m'apporte des satisfactions. & $\square_{1}$ \\
\hline EC022Q10NA & Cela fait bonne impression sur un curriculum vitae. & $\square_{1}$ \\
\hline EC022Q11NA & C'est nécessaire pour trouver un emploi. & $\square_{1}$ \\
\hline EC022Q12NA & Autre raison. & $\square_{1}$ \\
\hline
\end{tabular}

\begin{tabular}{|c|c|c|}
\hline \multirow[b]{2}{*}{ EC023 } & \multicolumn{2}{|c|}{$\begin{array}{l}\rightarrow \text { Uniquement si l'élève ne suit aucun « cours supplémentaires en mathématiques ». } \\
\text { Dans le cas contraire, passer cette question et aller directement à la question EC024. }\end{array}$} \\
\hline & \multicolumn{2}{|c|}{$\begin{array}{l}\text { Pourquoi ne suivez-vous pas de cours supplémentaires de mathématiques cette année scolaire-ci ? } \\
\text { (Sélectionnez toutes les réponses qui conviennent.) }\end{array}$} \\
\hline EC023Q01NA & Je n'ai pas besoin de cours supplémentaires de mathématiques. & $\square_{1}$ \\
\hline EC023Q02NA & Aucune des offres disponibles ne semblait convenir à mes besoins. & $\square_{1}$ \\
\hline EC023Q03NA & Il n'y a pas beaucoup de mes amis qui le font. & $\square_{1}$ \\
\hline EC023Q04NA & Je n'ai pas le temps. & $\square_{1}$ \\
\hline EC023Q05NA & Je n'ai pas de quoi les payer. & $\square_{1}$ \\
\hline EC023Q06NA & Mes professeurs de l'école s'y connaissent suffisamment. & $\square_{1}$ \\
\hline EC023Q07NA & Mes parents ne veulent pas. & $\square_{1}$ \\
\hline EC023Q08NA & Ils semblent trop chers pour ce qu'ils sont. & $\square_{1}$ \\
\hline EC023Q09NA & Mes professeurs disent que ce n'est pas utile. & $\square_{1}$ \\
\hline EC023Q10NA & Je n'ai jamais envisagé de prendre des cours supplémentaires de mathématiques. & $\square_{1}$ \\
\hline EC023Q11NA & Il n'y a pas de cours supplémentaires de mathématiques disponibles là où j'habite. & $\square_{1}$ \\
\hline EC023Q12NA & C'est ma famille qui m’aide. & $\square_{1}$ \\
\hline EC023Q13NA & Ce sont mes amis et de mes camarades de classe qui m'aident. & $\square_{1}$ \\
\hline
\end{tabular}




\section{SECTION C COURS SUPPLÉMENTAIRES DE <LANGUE DE L'ÉVALUATION>}

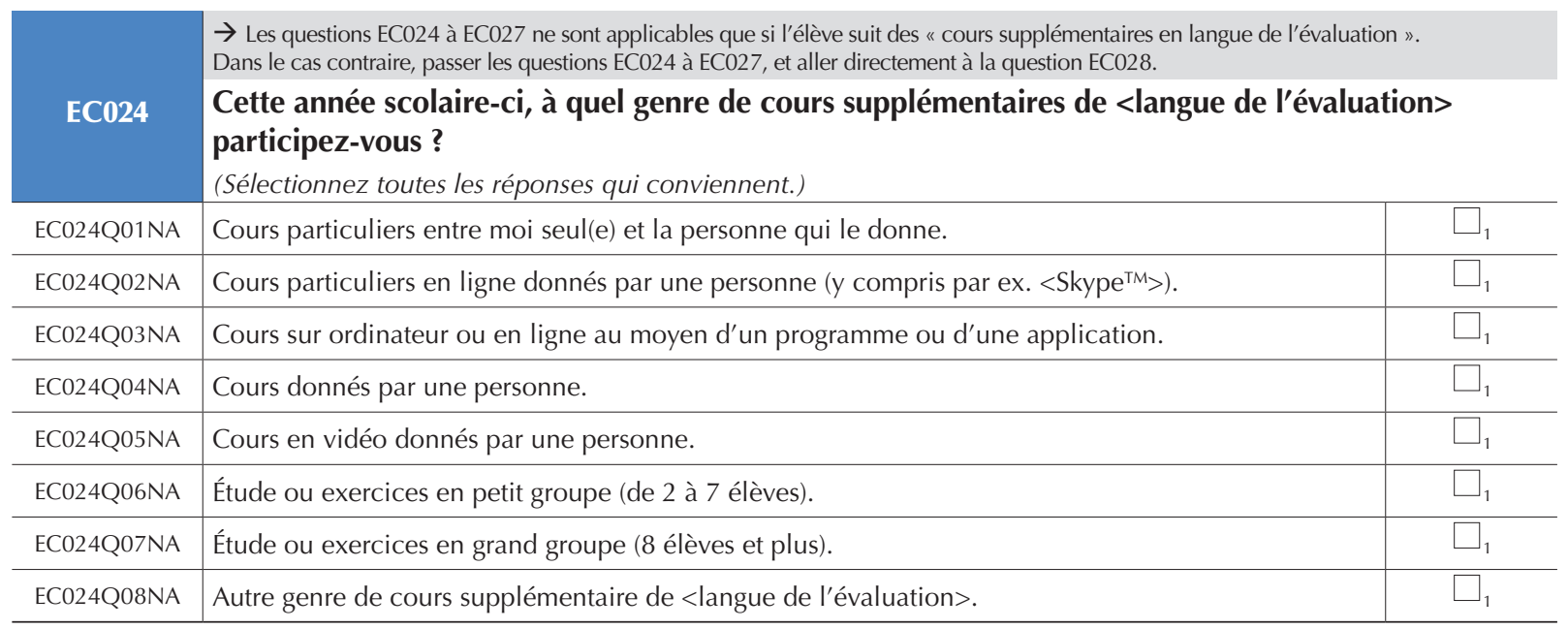

\begin{tabular}{c|l|c}
\multirow{2}{*}{ EC026 } & $\begin{array}{l}\rightarrow \text { Les questions EC024 à EC027 ne sont applicables que si l'élève suit des « cours supplémentaires en langue de l'évaluation ». } \\
\text { Dans le cas contraire, passer les questions EC024 à EC027, et aller directement à la question EC028. } \\
\text { Où suivez-vous ces cours supplémentaires de <langue de l'évaluation> ? } \\
\text { (Sélectionnez toutes les réponses qui conviennent.) }\end{array}$ \\
\hline EC026Q01NA & Dans mon établissement scolaire habituel. & $\square$ \\
\hline EC026Q02NA & Ailleurs, c'est-à-dire en dehors de mon établissement scolaire habituel. & $\square_{1}$ \\
\hline
\end{tabular}

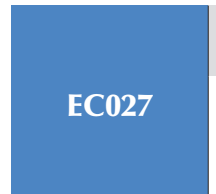

EC027Q01NA EC027Q02NA

EC027Q03NA

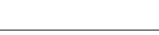

$\rightarrow$ Les questions EC024 à EC027 ne sont applicables que si l'élève suit des « cours supplémentaires en langue de l'évaluation ». Dans le cas contraire, passer les questions EC024 à EC027, et aller directement à la question EC028.

Parmi les descriptions suivantes, laquelle ou lesquelles correspond(ent) au professeur ou tuteur qui vous donne des cours supplémentaires de <langue de l'évaluation>?

(Sélectionnez toutes les réponses qui conviennent)

EC027Q04NA

Ce professeur est l'un des professeurs de mon école que j'ai cette année.

Ce professeur enseigne régulièrement à des élèves de mon âge dans mon école mais ce n'est pas un de mes professeurs.

Ce professeur travaille principalement pour une société ou une organisation spécialisée dans les cours supplémentaires.

Ce professeur n'est pas un enseignant qualifié (par ex. un étudiant).

\section{SECTION D PARCOURS SCOLAIRE}

EC028

Avez-vous déjà suivi des cours supplémentaires plus tôt dans votre scolarité ?

(Sélectionnez une réponse par ligne.)

\begin{tabular}{l|l|c|c}
\hline \multicolumn{2}{l|}{} & Oui & Non \\
\hline EC028Q01NA & Au <niveau CITE 0 $>$ & $\square_{1}$ & $\square_{2}$ \\
\hline EC028Q02NA & Au <niveau CITE 1 $>$ & $\square_{1}$ & $\square_{2}$ \\
\hline EC028Q03NA & Au <niveau CITE 2 $>$ & $\square_{1}$ & $\square_{2}$
\end{tabular}

Il s'agit ici d'une question filtre. La question EC029 n'est applicable que si I'une quelconque des réponses à la question EC028 est supérieure à 0. 

dans sa scolarité).

Au total, pendant combien d'années avez-vous suivi des cours supplémentaires?

(Sélectionnez votre réponse dans le menu déroulant.)

Nombre d'années: Sélectionnez une option $\mathbf{\nabla}$

Option $A$
Option $B$
Option C
Option ...

Menu déroulant : options de réponse 0-16

Dans votre famille, les personnes suivantes vous aident-elles régulièrement à faire vos devoirs ou à étudier?

(Sélectionnez une réponse par ligne.)

\begin{tabular}{|c|c|c|c|}
\hline & & Oui & Non \\
\hline EC030Q01NA & Votre mère ou une autre femme responsable de vous. & $\square_{1}$ & $\square_{2}$ \\
\hline EC030Q02NA & Votre père ou un autre homme responsable de vous. & $\square_{1}$ & $\square_{2}$ \\
\hline EC030Q03NA & Vos frère(s) / sœur(s). & $\square_{1}$ & $\square_{2}$ \\
\hline EC030Q04NA & Vos grands-parents. & $\square_{1}$ & $\square_{2}$ \\
\hline EC030Q05NA & D'autres membres de la famille. & $\square_{1}$ & $\square_{2}$ \\
\hline EC030Q06NA & Personne. & $\square_{1}$ & $\square_{2}$ \\
\hline EC030Q07NA & Quelqu'un d'autre. & $\square_{1}$ & $\square_{2}$ \\
\hline
\end{tabular}

\section{EC031}

EC031Q01TA

EC031Q02TA

EC031Q03TA

Vous est-il arrivé de changer d'école durant votre scolarité de <niveau CITE 1> ?

(Sélectionnez une réponse.)

Non, j'ai fait toute ma scolarité de <niveau CITE $1>$ dans la même école.

Oui, j'ai changé d'école une fois.

Oui, j'ai changé d'école deux fois ou plus.

EC032

EC032Q01TA

EC032Q02TA

EC032Q03TA

Oui, j'ai changé d'école deux fois ou plus.

(Sélectionnez une réponse.)

Oui, j'ai changé d'école une fois.

Vous est-il arrivé de changer d'école durant votre scolarité de <niveau CITE 2> ?

Non, j'ai fait toute ma scolarité de <niveau CITE 2> dans la même école.

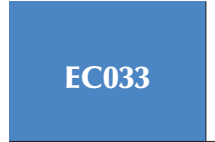

Avez-vous déjà changé de <programme d'études> ?

(<exemple>)

(Sélectionnez une réponse.)

\begin{tabular}{l|l|c} 
EC033Q01NA & Non. & $\square$ \\
\hline EC033Q02NA & Oui, j'ai changé de<programme d'études> une fois. & $\square$, \\
\hline EC033Q03NA & Oui, j'ai changé de <programme d'études> deux fois ou plus. & $\square_{2}$ \\
\hline
\end{tabular}


QUESTIONNAIRE PORTANT SUR LA MAÎTRISE DES TECHNOLOGIES

DE L'INFORMATION ET DE LA COMMUNICATION

(Option internationale)

\section{Version source pour la campagne définitive}

Le questionnaire portant sur la maîtrise des technologies de l'information et de la communication (TIC) se compose d'une série de questions sur l'accès des élèves aux TIC ainsi que sur leur utilisation de l'informatique et leurs attitudes à cet égard. Les élèves peuvent remplir ce questionnaire en 5 minutes environ, après avoir répondu au questionnaire " Élève ».

Ce questionnaire est destiné à recueillir des renseignements sur :

- L'accès aux TIC

- L'utilisation générale de l'informatique

- L'utilisation des TIC en dehors de l'école

- L'utilisation des TIC à l'école

- Les attitudes à l'égard de l'informatique

Les questions suivantes portent sur différents aspects en rapport avec les médias et appareils numériques, y compris les ordinateurs de bureau, les ordinateurs portables, les agendas électroniques, les smartphones, les tablettes tactiles, les téléphones portables sans connexion à Internet, les consoles de jeux et les télévisions connectées à Internet.

\section{IC001}

À la maison, avez-vous la possibilité d'utiliser les équipements suivants ?

(Sélectionnez une réponse par ligne.)

\begin{tabular}{|c|c|c|c|c|}
\hline & & $\begin{array}{l}\text { Oui, et } \\
\text { je l'utilise }\end{array}$ & $\begin{array}{c}\text { Oui, mais } \\
\text { je ne l'utilise pas }\end{array}$ & Non \\
\hline IC001Q01TA & Un ordinateur de bureau & $\square_{1}$ & $\square_{2}$ & $\square_{3}$ \\
\hline IC001Q02TA & Un ordinateur portable ou un agenda électronique & $\square_{1}$ & $\square_{2}$ & $\square_{3}$ \\
\hline IC001Q03TA & Une $<$ tablette tactile $>$ (par ex. un $<$ iPad $^{\circ}>$ ou un $<$ BlackBerry $^{\circ}$ PlayBook $^{\mathrm{TM}}>$ ) & $\square_{1}$ & $\square_{2}$ & $\square_{3}$ \\
\hline IC001Q04TA & Une connexion à Internet & $\square_{1}$ & $\square_{2}$ & $\square_{3}$ \\
\hline IC001Q05TA & 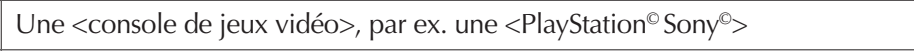 & $\square_{1}$ & $\square_{2}$ & $\square_{3}$ \\
\hline IC001Q06TA & Un <téléphone portable> (sans connexion à Internet) & $\square_{1}$ & $\square_{2}$ & $\square_{3}$ \\
\hline IC001Q07TA & Un < téléphone portable> (avec connexion à Internet) & $\square_{1}$ & $\square_{2}$ & $\square_{3}$ \\
\hline IC001Q08TA & Un lecteur de musique portable (lecteur $\mathrm{Mp} 3$ ou $\mathrm{Mp} 4$, un $\mathrm{iPod}^{\circ}$ ou équivalent) & $\square_{1}$ & $\square_{2}$ & $\square_{3}$ \\
\hline IC001Q09TA & Une imprimante & $\square_{1}$ & $\square_{2}$ & $\square_{3}$ \\
\hline IC001Q10TA & Une clé USB & $\square_{1}$ & $\square_{2}$ & $\square_{3}$ \\
\hline IC001Q11TA & Un $<$ lecteur électronique $>$, par ex. un $<$ Amazon $^{\odot}$ Kindlle $^{T M}>$ & $\square_{1}$ & $\square_{2}$ & $\square_{3}$ \\
\hline
\end{tabular}

IC009

À l'école, avez-vous la possibilité d'utiliser les équipements suivants ?

(Sélectionnez une réponse par ligne.)

\begin{tabular}{|c|c|c|c|c|}
\hline & & $\begin{array}{l}\text { Oui, et } \\
\text { je l'utilise }\end{array}$ & $\begin{array}{l}\text { Oui, mais } \\
\text { je ne l'utilise pas }\end{array}$ & Non \\
\hline IC009Q01TA & Un ordinateur de bureau & $\square_{1}$ & $\square_{2}$ & $\square_{3}$ \\
\hline IC009Q02TA & Un ordinateur portable ou un agenda électronique & $\square_{1}$ & $\square_{2}$ & $\square_{3}$ \\
\hline IC009Q03TA & Une $<$ tablette tactile $>$ (par ex. un $<$ iPad $^{\odot}>$ ou un $<$ BlackBerry $^{\odot}$ PlayBook $^{\mathrm{TM}}>$ ) & $\square_{1}$ & $\square_{2}$ & $\square_{3}$ \\
\hline IC009Q05NA & Des ordinateurs de l'école connectés à Internet & $\square_{1}$ & $\square_{2}$ & $\square_{3}$ \\
\hline IC009Q06NA & Une connexion Internet accessible par un réseau sans fil & $\square_{1}$ & $\square_{2}$ & $\square_{3}$ \\
\hline IC009Q07NA & $\begin{array}{l}\text { Un espace de stockage pour des données scolaires, par ex. un dossier } \\
\text { où sauvegarder mes fichiers }\end{array}$ & $\square_{1}$ & $\square_{2}$ & $\square_{3}$ \\
\hline IC009Q08TA & Une clé USB & $\square_{1}$ & $\square_{2}$ & $\square_{3}$ \\
\hline IC009Q09TA & Un $<$ lecteur électronique $>$, par ex. un $<$ Amazon $^{\odot}$ Kindle $^{\mathrm{TM}}>$ & $\square_{1}$ & $\square_{2}$ & $\square_{3}$ \\
\hline IC009Q10NA & Un vidéoprojecteur, par exemple pour la présentation d'un diaporama & $\square_{1}$ & $\square_{2}$ & $\square_{3}$ \\
\hline IC009Q11NA & Un tableau blanc interactif, par ex. $<$ SmartBoard $^{\circledR}>$ & $\square_{1}$ & $\square_{2}$ & $\square_{3}$ \\
\hline
\end{tabular}


ICOO2

IC002Q01NA
À quel âge avez-vous utilisé un appareil numérique pour la première fois ?

(Pensez à plusieurs types d'appareils numériques comme, par exemple, des ordinateurs de bureau, des ordinateurs portables, des agendas électroniques, des smartphones, des tablettes tactiles, des téléphones portables sans connexion à Internet, des consoles de jeux, ou des télévisions connectées à Internet.)

(Sélectionnez une réponse.)

$\rightarrow$ Si l'élève choisit l'option de réponse « Je n'ai jamais utilisé d'appareil numérique », il peut s'arrêter ici, dans la mesure où les questions suivantes ne seront pas pertinentes pour lui.

IC003 IC003Q01TA
À quel âge avez-vous utilisé un ordinateur pour la première fois ?

(Sélectionnez une réponse.)
IC004 IC004Q01T
À quel âge vous êtes-vous servi d'Internet pour la première fois ?

(Sélectionnez une réponse.)
IC005

IC005Q01TA $\rightarrow$ Uniquement si l'élève a choisi une des options de réponse 1 à 4 à la question IC004.

Un jour de semaine ordinaire, combien de temps utilisez-vous Internet à l'école?

(Ne sélectionnez qu'une seule réponse.)

\begin{tabular}{c|c|c|c|c|c|c} 
Pas du tout & $\begin{array}{c}\text { De } 1 \\
\text { à } 30 \text { minutes } \\
\text { par jour }\end{array}$ & $\begin{array}{c}\text { De } 31 \\
\text { à } 60 \text { minutes } \\
\text { par jour }\end{array}$ & $\begin{array}{c}\text { Entre } 1 \\
\text { et } 2 \text { heures } \\
\text { par jour }\end{array}$ & $\begin{array}{c}\text { Entre } 2 \\
\text { et } 4 \text { heures } \\
\text { par jour }\end{array}$ & $\begin{array}{c}\text { Entre } 4 \\
\text { et } 6 \text { heures } \\
\text { par jour }\end{array}$ & $\begin{array}{c}\text { Plus de } \\
6 \text { heures } \\
\text { par jour }\end{array}$ \\
\hline$\square_{1}$ & $\square_{2}$ & $\square_{3}$ & $\square_{4}$ & $\square_{5}$ & $\square_{6}$ & $\square_{7}$ \\
\hline
\end{tabular}

IC006

IC006Q01TA

$\rightarrow$ Uniquement si l'élève a choisi une des options de réponse 1 à 4 à la question IC004.

Un jour de semaine ordinaire, combien de temps utilisez-vous Internet en dehors de l'école ?

(Ne sélectionnez qu'une seule réponse.)

\begin{tabular}{c|c|c|c|c|c|c|c} 
& Pas du tout & $\begin{array}{c}\text { De } 1 \\
\text { à } 30 \text { minutes } \\
\text { par jour }\end{array}$ & $\begin{array}{c}\text { De } 31 \\
\text { à } 60 \text { minutes } \\
\text { par jour }\end{array}$ & $\begin{array}{c}\text { Entre } 1 \\
\text { et } 2 \text { heures } \\
\text { par jour }\end{array}$ & $\begin{array}{c}\text { Entre } 2 \\
\text { et } 4 \text { heures } \\
\text { par jour }\end{array}$ & $\begin{array}{c}\text { Entre } 4 \\
\text { et } 6 \text { heures } \\
\text { par jour }\end{array}$ & $\begin{array}{c}\text { Plus de } \\
\mathbf{6} \text { heures } \\
\text { par jour }\end{array}$ \\
\hline & $\square_{1}$ & $\square_{2}$ & $\square_{3}$ & $\square_{4}$ & $\square_{5}$ & $\square_{6}$ & $\square_{7}$ \\
\hline
\end{tabular}

IC007

IC007Q01TA

$\rightarrow$ Uniquement si l'élève a choisi une des options de réponse 1 à 4 à la question IC004.

Un jour de week-end ordinaire, combien de temps utilisez-vous Internet en dehors de l'école?

(Ne sélectionnez qu'une seule réponse.)

\begin{tabular}{|c|c|c|c|c|c|c|}
\hline Pas du tout & $\begin{array}{c}\text { De } 1 \\
\text { à } 30 \text { minutes } \\
\text { par jour }\end{array}$ & $\begin{array}{c}\text { De } 31 \\
\text { à } 60 \text { minutes } \\
\text { par jour }\end{array}$ & $\begin{array}{c}\text { Entre } 1 \\
\text { et } 2 \text { heures } \\
\text { par jour }\end{array}$ & $\begin{array}{c}\text { Entre } 2 \\
\text { et } 4 \text { heures } \\
\text { par jour }\end{array}$ & $\begin{array}{c}\text { Entre } 4 \\
\text { et } 6 \text { heures } \\
\text { par jour }\end{array}$ & $\begin{array}{l}\text { Plus de } \\
6 \text { heures } \\
\text { par jour }\end{array}$ \\
\hline$\square_{1}$ & $\square_{2}$ & $\square_{3}$ & $\square_{4}$ & $\square_{5}$ & $\square_{6}$ & $\square_{7}$ \\
\hline
\end{tabular}


En dehors de l'école, à quelle fréquence utilisez-vous un appareil numérique pour les activités suivantes?

(Sélectionnez une réponse par ligne.)

\begin{tabular}{|c|c|c|c|c|c|c|}
\hline & & $\begin{array}{c}\text { Jamais } \\
\text { ou presque } \\
\text { jamais }\end{array}$ & $\begin{array}{l}\text { Une ou } \\
\text { deux fois } \\
\text { par mois }\end{array}$ & $\begin{array}{c}\text { Une ou } \\
\text { deux fois } \\
\text { par semaine }\end{array}$ & $\begin{array}{l}\text { Presque } \\
\text { tous } \\
\text { les jours }\end{array}$ & $\begin{array}{l}\text { Tous } \\
\text { les jours }\end{array}$ \\
\hline IC008Q01TA & Jouer à des jeux à un seul joueur & $\square_{1}$ & $\square_{2}$ & $\square_{3}$ & $\square_{4}$ & $\square_{5}$ \\
\hline IC008Q02TA & $\begin{array}{l}\text { Jouer à des jeux à plusieurs (sur Internet } \\
\text { ou en réseau) }\end{array}$ & $\square_{1}$ & $\square_{2}$ & $\square_{3}$ & $\square_{4}$ & $\square_{5}$ \\
\hline IC008Q03TA & Utiliser le courrier électronique (e-mail) & $\square_{1}$ & $\square_{2}$ & $\square_{3}$ & $\square_{4}$ & $\square_{5}$ \\
\hline IC008Q04TA & $<$ Chatter en ligne $>$ (par ex. sur $<\mathrm{MSN}^{\circ}>$ ) & $\square_{1}$ & $\square_{2}$ & $\square_{3}$ & $\square_{4}$ & $\square_{5}$ \\
\hline IC008Q05TA & $\begin{array}{l}\text { Participer à des réseaux sociaux } \\
(\text { par ex. }<\text { Facebook }>,<\text { MySpace }>\text { ) }\end{array}$ & $\square_{1}$ & $\square_{2}$ & $\square_{3}$ & $\square_{4}$ & $\square_{5}$ \\
\hline IC008Q07NA & $\begin{array}{l}\text { Jouer à des jeux en ligne via des réseaux sociaux } \\
\left(\text { par ex. }<\text { Farmville }^{\circledR}>,<\text { The Sims Social }>\text { ) }\right.\end{array}$ & $\square_{1}$ & $\square_{2}$ & $\square_{3}$ & $\square_{4}$ & $\square_{5}$ \\
\hline IC008Q08TA & $\begin{array}{l}\text { Surfer sur Internet pour m'amuser (par ex. pour regarder } \\
\text { des vidéos sur }<\text { YouTube }^{\mathrm{TM}}>\text { ) }\end{array}$ & $\square_{1}$ & $\square_{2}$ & $\square_{3}$ & $\square_{4}$ & $\square_{5}$ \\
\hline IC008Q09TA & Suivre I'actualité sur Internet & $\square_{1}$ & $\square_{2}$ & $\square_{3}$ & $\square_{4}$ & $\square_{5}$ \\
\hline IC008Q10TA & $\begin{array}{l}\text { Obtenir des informations pratiques sur Internet } \\
\text { (par exemple le lieu et la date d'un événement) }\end{array}$ & $\square_{1}$ & $\square_{2}$ & $\square_{3}$ & $\square_{4}$ & $\square_{5}$ \\
\hline IC008Q11TA & $\begin{array}{l}\text { Télécharger de la musique, des films, des jeux } \\
\text { ou des logiciels à partir d'Internet }\end{array}$ & $\square_{1}$ & $\square_{2}$ & $\square_{3}$ & $\square_{4}$ & $\square_{5}$ \\
\hline IC008Q12TA & $\begin{array}{l}\text { Mettre en ligne mes propres créations pour } \\
\text { les partager (par exemple de la musique, des poèmes, } \\
\text { des vidéos, des programmes informatiques) }\end{array}$ & $\square_{1}$ & $\square_{2}$ & $\square_{3}$ & $\square_{4}$ & $\square_{5}$ \\
\hline IC008Q13NA & $\begin{array}{l}\text { Télécharger de nouvelles applications } \\
\text { sur un appareil mobile }\end{array}$ & $\square_{1}$ & $\square_{2}$ & $\square_{3}$ & $\square_{4}$ & $\square_{5}$ \\
\hline
\end{tabular}

En dehors de l'école, à quelle fréquence utilisez-vous des appareils numériques pour les activités suivantes?

(Sélectionnez une réponse par ligne.)

\begin{tabular}{c|l|c|c|c|c|c} 
& \multicolumn{1}{|c|}{$\begin{array}{c}\text { Jamais } \\
\text { ou presque } \\
\text { jamais }\end{array}$} & $\begin{array}{c}\text { Une ou } \\
\text { deux fois } \\
\text { par mois }\end{array}$ & $\begin{array}{c}\text { Une ou } \\
\text { deux fois } \\
\text { par semaine }\end{array}$ & $\begin{array}{c}\text { Presque } \\
\text { tous } \\
\text { les jours }\end{array}$ & $\begin{array}{c}\text { Tous } \\
\text { les jours }\end{array}$ \\
\hline IC010Q01TA & $\begin{array}{l}\text { Surfer sur Internet pour le travail scolaire (pour } \\
\text { préparer une rédaction ou un exposé, par exemple) }\end{array}$ & $\square_{1}$ & $\square_{2}$ & $\square_{3}$ & $\square_{4}$ \\
\hline IC010Q02NA & $\begin{array}{l}\text { Surfer sur Internet quand jerevois mes cours, } \\
\text { pour trouver des explications par exemple }\end{array}$ & $\square_{1}$ & $\square_{2}$ & $\square_{3}$ & $\square_{4}$ \\
\hline IC010Q03TA & $\begin{array}{l}\text { Échanger des e-mails avec d'autres élèves à propos } \\
\text { du travail scolaire }\end{array}$ & $\square_{1}$ & $\square_{2}$ & $\square_{3}$ & $\square_{4}$ \\
\hline IC010Q04TA & $\begin{array}{l}\text { Communiquer par e-mail avec les professeurs } \\
\text { et rendre mes devoirs ou autres travaux }\end{array}$ & $\square_{1}$ & $\square_{2}$ & $\square_{3}$ & $\square_{4}$ \\
\hline IC010Q05NA & $\begin{array}{l}\text { Utiliser les réseaux sociaux (par ex. <Facebook>, } \\
\text { <MySpace>) pour communiquer avec d'autres élèves } \\
\text { à proposdutravail scolaire }\end{array}$ & $\square_{1}$ & $\square_{2}$ & $\square_{3}$ & $\square_{4}$ \\
\hline IC010Q06NA & $\begin{array}{l}\text { Utiliser les réseaux sociaux (par ex. <Facebook>, } \\
\text { <MySpace>) pour communiquer avec les professeurs }\end{array}$ & $\square_{1}$ & $\square_{2}$ & $\square_{3}$ & $\square_{4}$ \\
\hline IC010Q07TA & $\begin{array}{l}\text { Télécharger ou consulter des documents } \\
\text { sur le site web de mon école (par ex.des horaires } \\
\text { ou documents de cours) }\end{array}$ & $\square_{1}$ & $\square_{2}$ & $\square_{3}$ & $\square_{4}$ \\
\hline IC010Q08TA & $\begin{array}{l}\text { Consulter le site web de l'école pour aller } \\
\text { aux renseignements (par exemple les absences } \\
\text { de professeurs) }\end{array}$ & $\square_{1}$ & $\square_{2}$ & $\square_{3}$ & $\square_{5}$ \\
\hline IC010Q09NA & Faire mes devoirs sur ordinateur & $\square_{1}$ & $\square_{2}$ & $\square_{3}$ & $\square_{4}$ \\
\hline IC010Q10NA & Faire mes devoirs sur un appareil mobile & $\square_{1}$ & $\square_{2}$ & $\square_{3}$ & $\square_{4}$ \\
\hline IC010Q11NA & $\begin{array}{l}\text { Télécharger des applications éducatives } \\
\text { sur un appareil mobile }\end{array}$ & $\square_{5}$ \\
\hline IC010Q12NA & $\begin{array}{l}\text { Télécharger des applications éducatives en rapport } \\
\text { avec les sciences sur un appareil mobile }\end{array}$ & $\square_{1}$ & $\square_{2}$ & $\square_{3}$ & $\square_{5}$ \\
\hline
\end{tabular}


IC011

À l'école, à quelle fréquence utilisez-vous des appareils numériques pour les activités suivantes ? (Sélectionnez une réponse par ligne.)

\begin{tabular}{|c|c|c|c|c|c|c|}
\hline & & $\begin{array}{l}\text { Jamais } \\
\text { ou presque } \\
\text { jamais }\end{array}$ & $\begin{array}{l}\text { Une } \\
\text { ou deux fois } \\
\text { par mois }\end{array}$ & $\begin{array}{l}\text { Une } \\
\text { ou deux fois } \\
\text { par semaine }\end{array}$ & $\begin{array}{l}\text { Presque } \\
\text { tous } \\
\text { les jours }\end{array}$ & $\begin{array}{l}\text { Tous } \\
\text { les jours }\end{array}$ \\
\hline IC011Q01TA & $<$ Chatter en ligne > à l'école & $\square_{1}$ & $\square_{2}$ & $\square_{3}$ & & $\square_{5}$ \\
\hline IC011Q02TA & Utiliser le courrier électronique (e-mail) & & & $\square_{3}$ & & \\
\hline IC011Q03TA & Surfer sur Internet pour un travail scolaire & & & $\square_{3}$ & & $\square_{5}$ \\
\hline IC011Q04TA & $\begin{array}{l}\text { Télécharger, consulter des documents sur } \\
\text { le site web de l'école }\left(I^{\prime}<\text { intranet> par ex.) }\right. \\
\text { ou y déposer des fichiers }\end{array}$ & & & & & \\
\hline IC011Q05TA & $\begin{array}{l}\text { Déposer mes travaux sur le site web } \\
\text { de l'école }\end{array}$ & & & & & \\
\hline IC011Q06TA & Utiliser des logiciels de simulation à l'école & $\square_{1}$ & $\square_{2}$ & $\square_{3}$ & & $\square_{5}$ \\
\hline IC011Q07TA & $\begin{array}{l}\text { Faire des exercices (par ex. pour le cours de } \\
\text { langue étrangère ou celui de mathématiques) }\end{array}$ & & & $\square_{3}$ & & $\square_{5}$ \\
\hline IC011Q08TA & $\begin{array}{l}\text { Faire mes devoirs sur un ordinateur } \\
\text { de l'école }\end{array}$ & $\square_{1}$ & $\square_{2}$ & $\square_{3}$ & $\square_{4}$ & $\square_{5}$ \\
\hline IC011Q09TA & $\begin{array}{l}\text { Utiliser les ordinateurs de l'école pour un } \\
\text { travail de groupe ou pour communiquer avec } \\
\text { d'autres élèves }\end{array}$ & $\square_{1}$ & $\square_{2}$ & $\square_{3}$ & $\square_{4}$ & $\square_{5}$ \\
\hline
\end{tabular}

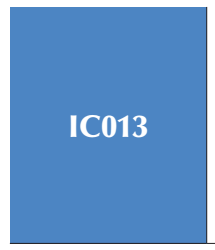

Pensez à l'expérience que vous avez des médias numériques et des appareils numériques. Dans quelle mesure êtes-vous d'accord ou non avec les affirmations suivantes?

(Pensez à plusieurs types d'appareils numériques comme, par exemple, des ordinateurs de bureau, des ordinateurs portables, des agendas électroniques, des smartphones, des tablettes tactiles, des téléphones portables sans connexion à Internet, des consoles de jeux, ou des télévisions connectées à Internet.)

(Sélectionnez une réponse par ligne.)

\begin{tabular}{c|l|c|c|c|c}
\multicolumn{1}{l|}{} & $\begin{array}{c}\text { Pas du tout } \\
\text { d'accord }\end{array}$ & $\begin{array}{c}\text { Pas } \\
\text { d'accord }\end{array}$ & $\begin{array}{c}\text { Tout à fait } \\
\text { D'accord } \\
\text { d'accord }\end{array}$ \\
\hline IC013Q01NA & $\begin{array}{l}\text { Quand j'utilise des appareils numériques, je ne vois } \\
\text { pas le temps passer. }\end{array}$ & $\square_{1}$ & $\square_{2}$ & $\square_{3}$ & $\square_{4}$ \\
\hline IC013Q04NA & $\begin{array}{l}\text { Internet est une formidable source pour trouver } \\
\text { QQQQles informations qui m'intéressent (par ex. } \\
\text { actualités, sports, dictionnaire). }\end{array}$ & $\square_{1}$ & $\square_{2}$ & $\square_{3}$ \\
\hline IC013Q05NA & Les réseaux sociaux sur Internet sont très utiles. & $\square_{1}$ & $\square_{2}$ & $\square_{3}$ & $\square_{4}$ \\
\hline IC013Q11NA & $\begin{array}{l}\text { Je suis toujours impatient(e) de découvrir de nouveaux } \\
\text { appareils ou applications numériques. }\end{array}$ & $\square_{1}$ & $\square_{2}$ & $\square_{3}$ & $\square_{4}$ \\
\hline IC013Q12NA & Cela m'énerve quand je n'ai pas d'accès à Internet. & $\square_{1}$ & $\square_{2}$ & $\square_{3}$ & $\square_{4}$ \\
\hline IC013Q13NA & J'aime utiliser des appareils numériques. & $\square_{1}$ & $\square_{2}$ & $\square_{3}$ & $\square 4$ \\
\hline
\end{tabular}




\begin{tabular}{|c|c|c|c|c|c|}
\hline \multirow[t]{2}{*}{ IC014 } & \multicolumn{5}{|c|}{$\begin{array}{l}\text { Pensez à l'expérience que vous avez des médias numériques et des appareils numériques. } \\
\text { Dans quelle mesure êtes-vous d'accord ou non avec les affirmations suivantes? } \\
\text { (Pensez à plusieurs types d'appareils numériques comme, par exemple, des ordinateurs de bureau, des ordinateurs } \\
\text { portables, des agendas électroniques, des smartphones, des tablettes tactiles, des téléphones portables sans connexion } \\
\text { à Internet, des consoles de jeux, ou des télévisions connectées à Internet.) } \\
\text { (Sélectionnez une réponse par ligne.) }\end{array}$} \\
\hline & & $\begin{array}{l}\text { Pas du tout } \\
\text { d'accord }\end{array}$ & $\begin{array}{c}\text { Pas } \\
\text { d'accord }\end{array}$ & D'accord & $\begin{array}{l}\text { Tout à fait } \\
\text { d'accord }\end{array}$ \\
\hline IC014Q03NA & $\begin{array}{l}\text { Je me sens à l'aise dans I'utilisation des appareils } \\
\text { numériques que je connais moins bien. }\end{array}$ & $\square_{1}$ & $\square_{2}$ & $\square_{3}$ & $\square_{4}$ \\
\hline IC014Q04NA & $\begin{array}{l}\text { Si mes amis ou ma famille veulent acheter } \\
\text { de nouveaux appareils ou applications numériques, } \\
\text { je peux les conseiller. }\end{array}$ & $\square_{1}$ & $\square_{2}$ & $\square 3$ & $\square_{4}$ \\
\hline IC014Q06NA & $\begin{array}{l}\text { Je me sens à l'aise dans I'utilisation des appareils } \\
\text { numériques à la maison. }\end{array}$ & $\square_{1}$ & $\square_{2}$ & $\square_{3}$ & $\square_{4}$ \\
\hline IC014Q08NA & $\begin{array}{l}\text { Quand je rencontre des problèmes avec un appareil } \\
\text { numérique, je pense pouvoir les résoudre. }\end{array}$ & $\square_{1}$ & $\square_{2}$ & $\square_{3}$ & $\square_{4}$ \\
\hline IC014Q09NA & $\begin{array}{l}\text { Si mes amis ou ma famille rencontrent un problème } \\
\text { avec un appareil numérique, je peux les aider. }\end{array}$ & $\square_{1}$ & $\square_{2}$ & $\square_{3}$ & $\square_{4}$ \\
\hline
\end{tabular}

IC015

Pensez à l'expérience que vous avez des médias numériques et des appareils numériques. Dans quelle mesure êtes-vous d'accord ou non avec les affirmations suivantes ?

(Sélectionnez une réponse par ligne.)

\begin{tabular}{|c|c|c|c|c|c|}
\hline & & $\begin{array}{l}\text { Pas du tout } \\
\text { d'accord }\end{array}$ & $\begin{array}{c}\text { Pas } \\
\text { d'accord }\end{array}$ & D'accord & $\begin{array}{l}\text { Tout à fait } \\
\text { d'accord }\end{array}$ \\
\hline IC015Q02NA & $\begin{array}{l}\text { Si j'ai besoin d'un nouveau logiciel, je l'installe } \\
\text { moi-même. }\end{array}$ & $\square_{1}$ & & & \\
\hline IC015Q03NA & $\begin{array}{l}\text { Je lis de la documentation sur les appareils numériques } \\
\text { afin d'être autonome. }\end{array}$ & $\square_{1}$ & & & \\
\hline IC015Q05NA & J'utilise les appareils numériques comme j'en ai envie. & $\square_{1}$ & $\square_{2}$ & $\square_{3}$ & $\square$ \\
\hline IC015Q07NA & $\begin{array}{l}\text { Si j'ai un problème avec un appareil numérique, } \\
\text { je commence par essayerdele résoudre par moi même. }\end{array}$ & $\square_{1}$ & $\square_{2}$ & & \\
\hline IC015Q09NA & $\begin{array}{l}\text { Si j'ai besoin d'une nouvelle application, je la choisis } \\
\text { moi même. }\end{array}$ & $\square_{1}$ & $\square_{2}$ & & $\square_{4}$ \\
\hline
\end{tabular}

Pensez à l'expérience que vous avez des médias numériques et des appareils numériques. Dans quelle mesure êtes-vous d'accord ou non avec les affirmations suivantes ?

(Sélectionnez une réponse par ligne.)

\begin{tabular}{|c|c|c|c|c|c|}
\hline & & $\begin{array}{l}\text { Pas du tout } \\
\text { d'accord }\end{array}$ & $\begin{array}{c}\text { Pas } \\
\text { d'accord }^{\prime}\end{array}$ & D'accord & $\begin{array}{l}\text { Tout à fait } \\
\text { d'accord }\end{array}$ \\
\hline IC016Q01NA & $\begin{array}{l}\text { J'aime discuter avec mes amis des appareils } \\
\text { numériques afin d'en apprendre plus. }\end{array}$ & $\square_{1}$ & & & \\
\hline IC016Q02NA & $\begin{array}{l}\text { J'aime échanger des solutions à des problèmes liés } \\
\text { aux appareils numériques avec d'autres personnes } \\
\text { sur Internet. }\end{array}$ & $\square_{1}$ & & & \\
\hline IC016Q04NA & $\begin{array}{l}\text { J'aime être avec mes amis pour jouer sur l'ordinateur } \\
\text { et à des jeux vidéo avec eux. }\end{array}$ & $\square_{1}$ & & & \\
\hline IC016Q05NA & $\begin{array}{l}\text { J'aime partager des informations sur les appareils } \\
\text { numériques avec mes amis. }\end{array}$ & $\square_{1}$ & $\square_{2}$ & & \\
\hline IC016Q07NA & $\begin{array}{l}\text { J'en apprends beaucoup sur les médias numériques } \\
\text { en discutant avec mes amis et ma famille. }\end{array}$ & $\square_{1}$ & $\square_{2}$ & & $\square_{4}$ \\
\hline
\end{tabular}




\section{QUESTIONNAIRE PARENTS}

(Option internationale)

\section{Version source pour la campagne définitive}

Un questionnaire " Parents » est distribué à chaque élève participant à l'enquête. Environ 20 minutes sont nécessaires pour le remplir. Il est destiné à recueillir des renseignements sur :

- L'enfant et sa famille

- L'établissement de l'enfant

- Le parcours éducatif de l'enfant durant la petite enfance

- Le point de vue des parents sur les sciences et l'environnement

- La situation des parents

\section{SECTION A VOTRE FAMILLE}

\begin{tabular}{c|l|c}
\multicolumn{1}{c|}{ QA001 } & $\begin{array}{l}\text { Qui remplira ce questionnaire ? } \\
\text { (Cochez toutes les cases qui conviennent.) }\end{array}$ & $\square$ \\
\hline PA001Q01TA & La mère (ou une autre femme tenant lieu de mère) & $\square$ \\
\hline PA001Q02TA & Le père (ou un autre homme tenant lieu de père) & $\square$ \\
\hline PA001Q03TA & Une autre personne & $\square$, \\
\hline
\end{tabular}

\begin{tabular}{|c|c|c|c|c|c|}
\hline \multirow[t]{2}{*}{ PA002 } & \multicolumn{5}{|c|}{$\begin{array}{l}\text { Quand votre enfant avait environ } 10 \text { ans, se livrait-il( elle) aux activités suivantes? } \\
\text { (Cochez une case par ligne.) }\end{array}$} \\
\hline & & Très souvent & Régulièrement & Parfois & Jamais \\
\hline PA002Q01TA & $\begin{array}{l}\text { Regarder des programmes télévisés sur des thèmes } \\
\text { scientifiques }\end{array}$ & & & & \\
\hline PA002Q02TA & Lire des livres sur les découvertes scientifiques & & & & \\
\hline PA002Q03TA & Regarder, lire ou écouter de la science-fiction & & $\square_{2}$ & & \\
\hline PA002Q04TA & Surfer sur des sites Web à caractère scientifique & $\square$ & 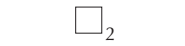 & & \\
\hline PA002Q05TA & Fréquenter un club de sciences & & & & \\
\hline PA002Q06NA & Jouer à des jeux de construction (par. ex Lego ${ }^{\circledR}$ ) & & & & \\
\hline PA002Q07NA & Démonter des appareils & $\square_{1}$ & $\square_{2}$ & $\square_{3}$ & \\
\hline PA002Q08NA & $\begin{array}{l}\text { Réparer des objets cassés, par ex. des jouets } \\
\text { électroniques cassés }\end{array}$ & & & & $\square$ \\
\hline PA002Q09NA & $\begin{array}{l}\text { Faire des expériences avec un coffret-jeu de science, } \\
\text { d'électronique ou de chimie, utiliser un microscope } \\
\text { ou un télescope }\end{array}$ & L & & & \\
\hline PA002Q10NA & Jouer à des jeux de science sur ordinateur & $\square_{1}$ & $\square_{2}$ & $\square_{3}$ & $\square_{4}$ \\
\hline
\end{tabular}


À quelle fréquence faites-vous (vous ou quelqu'un d'autre à la maison) les choses suivantes avec votre enfant?

(Cochez une case par ligne.)

\begin{tabular}{|c|c|c|c|c|c|c|}
\hline & & $\begin{array}{l}\text { Jamais } \\
\text { ou presque } \\
\text { jamais }\end{array}$ & $\begin{array}{l}\text { Une ou } \\
\text { deux fois } \\
\text { par an }\end{array}$ & $\begin{array}{l}\text { Une ou } \\
\text { deux fois } \\
\text { par mois }\end{array}$ & $\begin{array}{c}\text { Une ou } \\
\text { deux fois } \\
\text { par semaine }\end{array}$ & $\begin{array}{l}\text { Chaque jour } \\
\text { ou presque } \\
\text { chaque jour }\end{array}$ \\
\hline PA003Q01TA & $\begin{array}{l}\text { Discuter avec mon enfant de la qualité } \\
\text { de son travail scolaire }\end{array}$ & $\square_{1}$ & $\square_{2}$ & $\square_{3}$ & $\square_{4}$ & $\square_{5}$ \\
\hline PA003Q02TA & $\begin{array}{l}\text { Prendre le <repas principal > à table } \\
\text { avec mon enfant }\end{array}$ & $\square$ & $\square$ & $\square$ & $\square 4$ & $\square_{5}$ \\
\hline PA003Q03TA & $\begin{array}{l}\text { Passer du temps simplement à parler } \\
\text { avec mon enfant }\end{array}$ & $\square_{1}$ & $\square_{2}$ & $\square_{3}$ & $\square_{4}$ & $\square 5$ \\
\hline PA003Q04NA & $\begin{array}{l}\text { Aider mon enfant à faire ses devoirs } \\
\text { de sciences }\end{array}$ & $\square_{1}$ & $\square_{2}$ & $\square_{3}$ & $\square_{4}$ & $\square_{5}$ \\
\hline PA003Q05NA & $\begin{array}{l}\text { Discuter avec mon enfant de ses résultats } \\
\text { en sciences }\end{array}$ & $\square_{1}$ & $\square_{2}$ & $\square_{3}$ & $\square$ प & $\square_{5}$ \\
\hline PA003Q06NA & $\begin{array}{l}\text { Trouver du matériel de sciences } \\
\text { (par ex. des programmes, des logiciels, } \\
\text { des guides d'études, etc.) pour mon enfant }\end{array}$ & $\square_{1}$ & $\square_{2}$ & $\square_{3}$ & $\square_{4}$ & $\square_{5}$ \\
\hline PA003Q07NA & $\begin{array}{l}\text { Discuter avec mon enfant } \\
\text { de l'utilisation des sciences dans la vie } \\
\text { de tous les jours }\end{array}$ & $\square_{1}$ & $\square_{2}$ & $\square_{3}$ & $\square_{4}$ & $\square_{5}$ \\
\hline PA003Q08NA & $\begin{array}{l}\text { Discuter avec mon enfant des possibilités } \\
\text { de }<\text { professions à caractère scientifique }>\end{array}$ & $\square_{1}$ & $\square_{2}$ & $\square_{3}$ & $\square_{4}$ & $\square_{5}$ \\
\hline
\end{tabular}

En repensant à <l'année scolaire écoulée>, dans quelle mesure êtes-vous d'accord ou non avec les affirmations suivantes ?

(Cochez une case par ligne.)

\begin{tabular}{l|l|c|c|c|c}
\multicolumn{2}{l|}{} & $\begin{array}{c}\text { Pas du tout } \\
\text { d'accord }\end{array}$ & $\begin{array}{c}\text { Pas } \\
\text { d'accord }\end{array}$ & $\begin{array}{c}\text { Tout à fait } \\
\text { D'accord } \\
\text { d'accord }\end{array}$ \\
\hline PA004Q01NA & $\begin{array}{l}\text { Je me suis intéressé(e) aux activités scolaires } \\
\text { de mon enfant. }\end{array}$ & $\square_{1}$ & $\square_{2}$ & $\square_{3}$ & $\square_{4}$ \\
\hline PA004Q02NA & $\begin{array}{l}\text { J'ai encouragé mon enfant dans ses efforts et sa réussite } \\
\text { scolaire. }\end{array}$ & $\square_{1}$ & $\square_{2}$ & $\square_{3}$ & $\square_{4}$ \\
\hline PA004Q03NA & $\begin{array}{l}\text { J'ai soutenu mon enfant quand il a rencontré } \\
\text { des difficultés à l'école. }\end{array}$ & $\square_{1}$ & $\square_{2}$ & $\square_{3}$ & $\square_{4}$ \\
\hline PA004Q04NA & J'ai encouragé mon enfant à avoir confiance en lui. & $\square_{1}$ & $\square_{2}$ & $\square_{3}$ & $\square_{4}$ \\
\hline
\end{tabular}

\section{SECTION B L'ÉTABLISSEMENT DE VOTRE ENFANT}

Nous souhaiterions connaître les possibilités de choix que vous aviez, en tant que parents, au moment où vous avez choisi l'établissement que fréquente actuellement votre enfant.

PA005

PA005Q01TA

Parmi les énoncés suivants, lequel décrit le mieux les choix d'établissement qui sont offerts aux élèves là où vous vivez ?

(Ne cochez qu'une seule case.)

Il y a dans cette zone deux autres établissements, ou davantage, qui sont en concurrence avec l'établissement fréquenté actuellement par mon enfant.

Il y a dans cette zone un autre établissement qui est en concurrence avec l'établissement fréquenté actuellement par mon enfant.

Il n'y a dans cette zone aucun autre établissement en concurrence avec l'établissement fréquenté actuellement par mon enfant. 
Dans quelle mesure les critères suivants sont-ils importants pour choisir un établissement pour votre enfant?

(Cochez une case par ligne.)

\begin{tabular}{|c|c|c|c|c|c|}
\hline & & $\begin{array}{c}\text { Pas } \\
\text { important }\end{array}$ & $\begin{array}{c}\text { Assez } \\
\text { important }\end{array}$ & Important & $\begin{array}{c}\text { Très } \\
\text { important }\end{array}$ \\
\hline PA006Q01TA & L'établissement est proche de notre domicile. & $\square_{1}$ & $\square_{2}$ & $\square_{3}$ & $\square_{4}$ \\
\hline PA006Q02TA & L'établissement a bonne réputation. & $\square_{1}$ & $\square_{2}$ & $\square_{3}$ & $\square_{4}$ \\
\hline PA006Q03TA & $\begin{array}{l}\text { L'établissement propose des cours ou des matières } \\
\text { spécifiques. }\end{array}$ & $\square_{1}$ & $\square_{2}$ & $\square_{3}$ & $\square_{4}$ \\
\hline PA006Q04TA & $\begin{array}{l}\text { L'établissement adhère à une < « philosophie » religieuse> } \\
\text { particulière. }\end{array}$ & $\square_{1}$ & $\square_{2}$ & $\square_{3}$ & $\square_{4}$ \\
\hline PA006Q05TA & $\begin{array}{l}\text { L'établissement a une approche }<\text { pédagogique ou } \\
\text { didactique }>\text { particulière, par ex. }<\text { exemple }>\text {. }\end{array}$ & $\square_{1}$ & $\square_{2}$ & $\square_{3}$ & $\square_{4}$ \\
\hline PA006Q06TA & $\begin{array}{l}\text { D'autres membres de la famille fréquentent cet établissement, } \\
\text { ou l'ont fréquenté. }\end{array}$ & $\square_{1}$ & $\square_{2}$ & $\square_{3}$ & $\square_{4}$ \\
\hline PA006Q07TA & $\begin{array}{l}\text { Les < frais sont peu élevés }>\text { (par ex. droits de scolarité, coût } \\
\text { des manuels, frais de logement et de nourriture). }\end{array}$ & $\square_{1}$ & $\square_{2}$ & $\square_{3}$ & $\square_{4}$ \\
\hline PA006Q08TA & $\begin{array}{l}\text { L'établissement propose une <aide financière > (par ex. prêt } \\
\text { scolaire, bourse ou subvention). }\end{array}$ & $\square_{1}$ & $\square_{2}$ & $\square_{3}$ & $\square_{4}$ \\
\hline PA006Q09TA & $\begin{array}{l}\text { Il règne dans l'établissement une ambiance dynamique } \\
\text { et agréable. }\end{array}$ & $\square_{1}$ & $\square_{2}$ & $\square_{3}$ & $\square_{4}$ \\
\hline PA006Q10TA & Les résultats scolaires des élèves de l'établissement sont bons. & $\square_{1}$ & $\square_{2}$ & $\square_{3}$ & $\square_{4}$ \\
\hline PA006Q11TA & La sécurité y est bonne. & $\square_{1}$ & $\square_{2}$ & $\square_{3}$ & $\square_{4}$ \\
\hline
\end{tabular}

Nous souhaiterions connaître votre opinion sur l'établissement fréquenté par votre enfant.

PA007

\begin{tabular}{|c|c|c|c|c|c|}
\hline & & $\begin{array}{l}\text { Tout à fait } \\
\text { d'accord }\end{array}$ & $\mathrm{D}^{\prime}$ accord & $\begin{array}{c}\text { Pas } \\
\text { d'accord }\end{array}$ & $\begin{array}{l}\text { Pas du tout } \\
\text { d'accord }\end{array}$ \\
\hline PA007Q01TA & $\begin{array}{l}\text { La plupart des enseignants de l'établissement fréquenté } \\
\text { par mon enfant paraissent compétents et motivés. }\end{array}$ & $\square_{1}$ & $\square_{2}$ & $\square_{3}$ & $\square_{4}$ \\
\hline PA007Q02TA & Le niveau d'exigence est élevé dans cet établissement. & $\square_{1}$ & $\square_{2}$ & $\square_{3}$ & $\square_{4}$ \\
\hline PA007Q03TA & $\begin{array}{l}\text { Je suis satisfait(e) du contenu des cours et des méthodes } \\
\text { d'enseignement utilisées dans cet établissement. }\end{array}$ & $\square_{1}$ & $\square_{2}$ & $\square_{3}$ & $\square_{4}$ \\
\hline PA007Q04TA & Je suis satisfait(e) du climat de discipline de cet établissement. & $\square_{1}$ & $\square_{2}$ & $\square_{3}$ & $\square_{4}$ \\
\hline PA007Q05TA & $\begin{array}{l}\text { Les progrès de mon enfant sont suivis de près } \\
\text { par l'établissement. }\end{array}$ & & $\square_{2}$ & $\square_{3}$ & $\square_{4}$ \\
\hline PA007Q06TA & $\begin{array}{l}\text { Je reçois de l'établissement des informations régulières } \\
\text { et utiles sur les progrès scolaires de mon enfant. }\end{array}$ & $\square_{1}$ & $\square_{2}$ & $\square_{3}$ & $\square_{4}$ \\
\hline PA007Q07TA & Cet établissement donne une bonne formation aux élèves. & $\square_{1}$ & $\square_{2}$ & $\square_{3}$ & $\square_{4}$ \\
\hline PA007Q09NA & $\begin{array}{l}\text { Cet établissement m'invite régulièrement à participer } \\
\text { aux activités qu'il organise. }\end{array}$ & $\square_{1}$ & $\square_{2}$ & $\square_{3}$ & $\square_{4}$ \\
\hline PA007Q11NA & $\begin{array}{l}\text { L'établissement a mis en place des moyens de communication } \\
\text { efficaces entre l'école et les familles. }\end{array}$ & $\square_{1}$ & $\square_{2}$ & $\square_{3}$ & $\square_{4}$ \\
\hline PA007Q12NA & $\begin{array}{l}\text { Cet établissement implique les parents dans ses prises } \\
\text { de décision. }\end{array}$ & $\square_{1}$ & $\square_{2}$ & $\square 3$ & $\square 4$ \\
\hline PA007Q13NA & $\begin{array}{l}\text { Cet établissement propose des cours pour les parents (par ex. } \\
\text { des <cours d'alphabétisation }>\text { ) ou des programmes d'aide } \\
\text { aux familles (par ex. <aide en matière de santé, de nutrition>). }\end{array}$ & $\square_{1}$ & $\square_{2}$ & $\square_{3}$ & $\square 4$ \\
\hline PA007Q14NA & $\begin{array}{l}\text { Cet établissement fournit aux familles des informations } \\
\text { leur permettant d'aider les élèves dans leurs devoirs } \\
\text { et dans d'autres activités liées à l'école. }\end{array}$ & $\square_{1}$ & $\square_{2}$ & $\square_{3}$ & $\square_{4}$ \\
\hline PA007Q15NA & $\begin{array}{l}\text { Cet établissement collabore avec des <services à } \\
\text { la collectivité> destinés à renforcer les programmes scolaires } \\
\text { et le développement des élèves. }\end{array}$ & $\square_{1}$ & $\square_{2}$ & $\square_{3}$ & $\square$ प \\
\hline
\end{tabular}


Au cours de <l'année scolaire écoulée $>$, avez-vous pris part aux activités suivantes en rapport avec l'établissement de votre enfant?

(Cochez une case par ligne.)

\begin{tabular}{|c|c|c|c|c|}
\hline & & Oui & Non & $\begin{array}{l}\text { Non prévu } \\
\text { par l'établissement }\end{array}$ \\
\hline PA008Q01TA & $\begin{array}{l}\text { Discuter du comportement de mon enfant avec un professeur, } \\
\text { à ma demande. }\end{array}$ & $\square_{1}$ & $\square_{2}$ & $\square_{3}$ \\
\hline PA008Q02TA & $\begin{array}{l}\text { Discuter du comportement de mon enfant avec un professeur, à la demande } \\
\text { de ce dernier. }\end{array}$ & $\square_{1}$ & $\square_{2}$ & $\square_{3}$ \\
\hline PA008Q03TA & Discuter des progrès de mon enfant avec un professeur, à ma demande. & $\square_{1}$ & $\square_{2}$ & $\square_{3}$ \\
\hline PA008Q04TA & $\begin{array}{l}\text { Discuter des progrès de mon enfant avec l'un de ses professeurs, } \\
\text { à la demande de ce dernier. }\end{array}$ & $\square_{1}$ & $\square_{2}$ & $\square_{3}$ \\
\hline PA008Q05TA & $\begin{array}{l}\text { Participer à des activités liées à la gestion de l'école, par ex. faire partie d'un } \\
\text { comité consultatif de parents d'élèves ou du comité de direction de l'école. }\end{array}$ & $\square_{1}$ & $\square_{2}$ & $\square_{3}$ \\
\hline PA008Q06NA & $\begin{array}{l}\text { Me porter volontaire pour des tâches manuelles ou des activités parascolaires } \\
\text { (par ex. travaux de menuiserie, de jardinage ou d'entretien des bâtiments ou } \\
\text { de la cour ; pièce de théâtre, sports, excursion). }\end{array}$ & $\square_{1}$ & $\square_{2}$ & $\square_{3}$ \\
\hline PA008Q07NA & $\begin{array}{l}\text { Me porter volontaire pour participer à des activités scolaires (travailler } \\
\text { à la bibliothèque, à la médiathèque ou à la cantine, aider un professeur, } \\
\text { donner une conférence). }\end{array}$ & $\square_{1}$ & $\square_{2}$ & $\square_{3}$ \\
\hline PA008Q08NA & $\begin{array}{l}\text { Assister à une réunion programmée ou à des conférences } \\
\text { dans l'établissement. }\end{array}$ & $\square_{1}$ & $\square_{2}$ & $\square_{3}$ \\
\hline PA008Q09NA & $\begin{array}{l}\text { Échanger des idées avec les professeurs sur les stratégies pouvant aider } \\
\text { mon enfant dans ses apprentissages et ses devoirs à la maison. }\end{array}$ & $\square_{1}$ & $\square_{2}$ & $\square_{3}$ \\
\hline PA008Q10NA & $\begin{array}{l}\text { Échanger des idées avec les professeurs de mon enfant sur le rôle } \\
\text { des parents, le soutien familial et développement de l'enfant. }\end{array}$ & $\square_{1}$ & $\square_{2}$ & $\square_{3}$ \\
\hline
\end{tabular}

Au cours de <l'année scolaire écoulée $>$, vous a-t-il été difficile de participer à des activités de l'établissement de votre enfant pour l'une des raisons suivantes?

(Cochez une case par ligne.)

\begin{tabular}{l|l|c|c}
\multicolumn{2}{l|}{} & Oui & Non \\
\hline PA009Q01NA & Les heures des réunions ne me convenaient pas. & $\square_{1}$ & $\square_{2}$ \\
\hline PA009Q02NA & Je ne pouvais pas quitter mon travail. & $\square_{1}$ & $\square_{2}$ \\
\hline PA009Q03NA & Je n'avais personne pour garder mon ou mes enfants. & $\square_{1}$ & $\square_{2}$ \\
\hline PA009Q04NA & Le trajet jusqu'à l'école est dangereux & $\square_{1}$ \\
\hline PA009Q05NA & J'avais des problèmes de transport. & $\square_{2}$ \\
\hline PA009Q06NA & Je me sentais mal accueilli(e) dans cet établissement. & $\square_{1}$ \\
\hline PA009Q08NA & Je ne savais pas suffisamment la <langue d'évaluation>. & $\square_{2}$ \\
\hline PA009Q09NA & Je pensais que cela n'avait rien à voir avec le développement de mon enfant. & $\square_{2}$ \\
\hline PA009Q10NA & J'ignorais que je pouvais participer à des activités de l'établissement. & $\square_{1}$ \\
\hline PA009Q11NA & Mon enfant ne voulait pas que j'y participe. & $\square_{1}$ \\
\hline
\end{tabular}

Nous souhaitons en savoir plus sur l'interaction des parents avec les camarades d'école de leur enfant et avec le personnel de l'établissement.

(Cochez une case par ligne.)

\begin{tabular}{c|l|c|c|c|c}
\multicolumn{2}{l|}{} & $\mathbf{0}$ & $\mathbf{1}$ à 2 & $\mathbf{3}$ à 5 & $\mathbf{6}$ ou plus \\
\hline PA011Q01NA & $\begin{array}{l}\text { Combien de parents de camarades d'école de votre enfant } \\
\text { connaissez-vous? }\end{array}$ & $\square_{1}$ & $\square_{2}$ & $\square_{3}$ & $\square_{4}$ \\
\hline PA011Q02NA & $\begin{array}{l}\text { Combien de camarades d'école de votre enfant } \\
\text { connaissez-vous par leur prénom? }\end{array}$ & $\square_{1}$ & $\square_{2}$ & $\square_{3}$ & $\square_{4}$ \\
\hline PA011Q03NA & $\begin{array}{l}\text { Combien de membres du personnel de l'établissement } \\
\text { vous sentiriez-vous à l'aise de contacter si vous aviez } \\
\text { une question à propos de votre enfant ? }\end{array}$ & $\square_{1}$ & $\square_{2}$ & $\square_{3}$ & $\square_{4}$ \\
\hline
\end{tabular}




\section{SECTION C PARCOURS ÉDUCATIF DANS LA PETITE ENFANCE}

\section{PA014 À quel âge votre enfant est-t-il entré au <niveau CITE 1> ?}

PA014Q01NA Âge en années :

PA018

Avant d'entrer en <grade 1 du niveau CITE 1 , votre enfant a-t-il fréquenté de façon régulière une structure ayant l'un des objectifs suivants?

(Cochez une case par ligne.)

\begin{tabular}{|c|c|c|c|c|}
\hline & & Oui & Non & \\
\hline PA018Q01NA & Accueil et garde (par ex. <exemples nationaux $>$ ) & $\square_{1}$ & $\square_{2}$ & \begin{tabular}{|l}
$\mathrm{Si}$ "Oui " \\
veuillez répondre \\
aux questions 19 à 22 \\
\end{tabular} \\
\hline PA018Q02NA & $\begin{array}{l}\text { Développement éducatif de la petite enfance (par ex. } \\
<\text { <exemples nationaux }>\text { ) }\end{array}$ & $\square_{1}$ & $\square_{2}$ & $\begin{array}{l}\mathrm{Si} \text { "Oui », } \\
\text { veuillez répondre } \\
\text { aux questions } 23 \text { à } 26 \\
\end{array}$ \\
\hline PA018Q03NA & Enseignement pré-primaire (par ex. <exemples nationaux $>$ ) & $\square_{1}$ & $\square_{2}$ & $\begin{array}{l}\mathrm{Si} \text { "Oui », } \\
\text { veuillez répondre } \\
\text { aux questions } 27 \text { à } 30\end{array}$ \\
\hline
\end{tabular}

Si votre enfant n'a pas fréquenté de <structure d'éducation et d'accueil de la petite enfance $>$ avant le $<$ grade 1 du niveau CITE $1>$, veuillez passer à la question 32

\begin{tabular}{l|l|r}
\multicolumn{1}{c}{ PA019 } & $\begin{array}{l}\text { À quel(s) âge(s) votre enfant a-t-il fréquenté une <structure d'accueil et de garde> } \\
\text { avant d'entrer en <grade 1 du niveau CITE 1> ? } \\
\text { (Cochez toutes les cases qui conviennent.) }\end{array}$ & $\square_{1}$ \\
\hline PA019Q01NA & À moins d'un an & $\square_{1}$ \\
\hline PA019Q02NA & $\grave{A} 1$ an & $\square_{1}$ \\
\hline PA019Q03NA & $\grave{A} 2$ ans & $\square_{1}$ \\
\hline PA019Q04NA & $\grave{A} 3$ ans & $\square_{1}$ \\
\hline PA019Q05NA & $\grave{A} 4$ ans & $\square_{1}$ \\
\hline PA019Q06NA & $\grave{A} 5$ ans & $\square_{1}$ \\
\hline PA019Q07NA & $\grave{A} 6$ ans & $\square_{1}$ \\
\hline PA019Q08NA & $\grave{A} 7$ ans & \\
\hline
\end{tabular}

Qui s'est occupé de votre enfant et s'est chargé de son éducation dans la <structure d'accueil et de garde $>$ ?

(Cochez toutes les cases qui conviennent.)

PA020Q01NA $\quad$ Un frère ou une sœur mineurs de l'enfant (y compris les demi frères et demi-sœurs)

PA020Q02NA Un adulte membre de la famille de l'enfant (par ex. un des grands parents)

PA020Q03NA $\quad$ Un adulte sans formation sur la petite enfance et sans lien de parenté, par ex. baby-sitter, ami(e), voisin(e)

PA020Q04NA Un adulte formé sur la petite enfance (par ex. <professeur des écoles>, nourrice)

\begin{tabular}{|c|c|c|}
\hline PA021 & \multicolumn{2}{|c|}{$\begin{array}{l}\text { Où était située la <structure d'accueil et de garde> qui s'est occupée de votre enfant } \\
\text { ou s'est chargée de son éducation? } \\
\text { (Cochez toutes les cases qui conviennent.) }\end{array}$} \\
\hline PA021Q01NA & Au domicile de l'enfant & $\square_{1}$ \\
\hline PA021Q02NA & Au domicile d'une autre personne & $\square_{1}$ \\
\hline PA021Q03NA & Dans une institution de la petite enfance (par ex. <exemple national >) & $\square_{1}$ \\
\hline PA021Q04NA & À un autre endroit & $\square_{1}$ \\
\hline
\end{tabular}


PA022

PA022Q01NA

Quelle est la principale raison pour laquelle votre enfant a fréquenté une <structure d'accueil et de garde $>$ ?

(Ne cochez qu'une seule case.)

La fréquentation était obligatoire.

Nous ne pouvions pas nous occuper de l'enfant / je ne pouvais m'en occuper (par ex. travail, maladie).

Nous voulions / je voulais davantage de stimulation des apprentissages pour notre enfant / mon enfant (par ex. au niveau social, scolaire).

La plupart des autres enfants fréquentaient une $<$ structure d'accueil et de garde $>$.

\begin{tabular}{l|l} 
& \\
\hline e). & $\square_{2}$ \\
\hline & $\square_{3}$ \\
\hline
\end{tabular}

À quel(s) âge(s) votre enfant a-t-il fréquenté une <structure de développement éducatif

PA023 de la petite enfance $>$ avant d'entrer en $<$ grade 1 du niveau CITE $1>$ ?

(Cochez toutes les cases qui conviennent.)

PA023Q01NA À moins d'un an

PA023Q02NA $\grave{A} 1$ an

PA023Q03NA

PA023Q04NA

PA023Q05NA

PA023Q06NA À 5 ans

PA023Q07NA

PA023Q08NA

PA026

PA026Q01NA

Quelle est la principale raison pour laquelle votre enfant a fréquenté une <structure de développement éducatif de la petite enfance>?

(Ne cochez qu'une seule case.)

La fréquentation était obligatoire.

Nous ne pouvions pas nous occuper de l'enfant / je ne pouvais m'en occuper (par ex. travail, maladie).

Nous voulions / je voulais davantage de stimulation des apprentissages pour notre enfant / mon enfant (par ex. au niveau social, scolaire).

La plupart des autres enfants fréquentaient une <structure de développement éducatif de la petite enfance $>$.

\begin{tabular}{l|l}
$\square_{1}$ \\
$\square_{2}$ \\
$\square_{3}$ \\
\hline
\end{tabular}

\begin{tabular}{l|l|r}
\multicolumn{1}{c|}{ PA027 quel(s) âge(s) votre enfant a-t-il fréquenté une <structure d'enseignement pré-primaire> } \\
& $\begin{array}{l}\text { avant d'entrer en <grade 1 du niveau CITE 1 }>\text { ? } \\
\text { (Cochez toutes les cases qui conviennent.) }\end{array}$ & $\square_{1}$ \\
\hline PA027Q01NA & À moins d'un an & $\square_{1}$ \\
\hline PA027Q02NA & À 1 an & $\square_{1}$ \\
\hline PA027Q03NA & $\grave{A} 2$ ans & $\square_{1}$ \\
\hline PA027Q04NA & $\grave{A} 3$ ans & $\square_{1}$ \\
\hline PA027Q05NA & $\grave{A} 4$ ans & $\square_{1}$ \\
\hline PA027Q06NA & $\grave{A} 5$ ans & $\square_{1}$ \\
\hline PA027Q07NA & $\grave{A} 6$ ans & $\square_{1}$ \\
\hline PA027Q08NA & $\grave{A} 7$ ans &
\end{tabular}

Pour cette question, veuillez prendre en considération la dernière <structure d'enseignement pré-primaire $>$ que votre enfant a fréquentée avant d'entrer en <grade 1 du niveau CITE $1>$.

PA028 Quel type d'organisme proposait cette <structure d'enseignement pré-primaire> ?

PA028Q01NA (Ne cochez qu'une seule case.)

Organisme public avec un financement essentiellement public (par ex. <exemple national>)

Organisme privé avec un financement essentiellement public (par ex. <exemple national>)

Organisme privé avec un financement essentiellement privé (par ex. <exemple national>)

\begin{tabular}{|c}
$\square_{1}$ \\
$\square_{2}$ \\
$\square_{3}$
\end{tabular}


PA029

PA029Q01NA
Combien d'heures par semaine votre enfant fréquentait-il une <structure d'enseignement pré-primaire> à l'âge de trois ans ?

(Ne cochez qu'une seule case.)

\begin{tabular}{|l|c}
\hline 0 heure par semaine & $\square_{1}$ \\
\hline Jusqu'à 10 heures par semaine & $\square_{2}$ \\
\hline 11 à 20 heures par semaine & $\square_{3}$ \\
\hline 21 à 30 heures par semaine & $\square_{4}$ \\
\hline 31 à 40 heures par semaine & $\square_{5}$ \\
\hline 41 à 50 heures par semaine & $\square_{6}$ \\
\hline 51 heures par semaine ou plus & $\square_{7}$ \\
\hline
\end{tabular}

PA030
Quelle est la principale raison pour laquelle votre enfant a fréquenté une <structure d'enseignement pré-primaire> ?

(Ne cochez qu'une seule case.)

La fréquentation était obligatoire.

Nous ne pouvions pas nous occuper de l'enfant / je ne pouvais m'en occuper (par ex. travail, maladie).

Nous voulions / je voulais davantage de stimulation des apprentissages pour notre enfant / mon enfant (par ex. au niveau social, scolaire).

La plupart des autres enfants fréquentaient une $<$ structure d'enseignement pré-primaire $>$.

\section{SECTION D VOTRE POINT DE VUE SUR LES SCIENCES ET L'ENVIRONNEMENT}

Les questions qui suivent portent sur les <professions à caractère scientifique>. Nous entendons par <profession à caractère scientifique $>$ une profession pour laquelle il faut suivre des études supérieures dans un domaine scientifique, à l'université par exemple. Comme exemples de <professions à caractère scientifique>, citons le métier d'ingénieur (qui fait appel à la physique), de météorologue (qui fait appel aux sciences de la Terre), d'opticien (qui fait appel à la biologie et à la physique) et de médecin (qui fait appel aux sciences médicales).

\begin{tabular}{c|l|c|c}
\multicolumn{1}{c|}{ PA032 } & $\begin{array}{l}\text { Veuillez répondre aux questions ci-dessous. } \\
\text { (Cochez une case par ligne.) }\end{array}$ & \multicolumn{2}{c}{ Oui Non } \\
\hline PA032Q01TA & $\begin{array}{l}\text { Y a-t-il un membre de votre famille (y compris vous) qui exerce une <profession } \\
\text { à caractère scientifique }>\end{array}$ & $\square_{1}$ \\
\hline PA032Q02TA & Votre enfant manifeste-t-il de l'intérêt pour une <profession à caractère scientifique> ? & $\square_{1}$ \\
\hline PA032Q03TA & $\begin{array}{l}\text { Vous attendez-vous à ce que votre enfant s'oriente vers une <profession à caractère } \\
\text { scientifique }>\text { ? }\end{array}$ & $\square_{1}$ \\
\hline PA032Q04TA & $\begin{array}{l}\text { Votre enfant a-t-il manifesté de l'intérêt à l'idée de poursuivre des études scientifiques } \\
\text { quand il aura terminé <l'enseignement secondaire }>\end{array}$ & $\square_{1}$ & $\square_{2}$ \\
\hline PA032Q05TA & $\begin{array}{l}\text { Vous attendez-vous à ce que votre enfant poursuive des études scientifiques après } \\
\text { sa sortie de }<\text { l'enseignement secondaire }>\text { ? }\end{array}$ & $\square_{2}$ \\
\hline
\end{tabular}


PA033

Les sciences occupent une place importante dans l'enquête PISA. Nous souhaiterions connaître l'avis des parents d'élèves sur les sciences et les problèmes environnementaux.

Les questions suivantes portent sur vos opinions à propos des sciences.

Dans quelle mesure êtes-vous d'accord ou non avec les affirmations suivantes?

(Cochez une case par ligne.)

\begin{tabular}{c|l|c|c|c|c}
\multicolumn{2}{l|}{} & $\begin{array}{c}\text { Tout à fait } \\
\text { d'accord }\end{array}$ & D'accord & $\begin{array}{c}\text { Pas d'accord } \\
\text { Pas du tout } \\
\text { d'accord }\end{array}$ \\
\hline PA033Q02TA & $\begin{array}{l}\text { La <science au sens large> est importante pour } \\
\text { nous aider à comprendre le monde naturel. }\end{array}$ & $\square_{1}$ & $\square_{2}$ & $\square_{3}$ & $\square_{4}$ \\
\hline PA033Q06TA & La <science au sens large> est utile à la société. & $\square_{1}$ & $\square_{2}$ & $\square_{3}$ & $\square_{4}$ \\
\hline PA033Q07TA & $\begin{array}{l}\text { La <science au sens large> a beaucoup } \\
\text { d'importance à mes yeux. }\end{array}$ & $\square_{1}$ & $\square_{2}$ & $\square_{3}$ & $\square_{4}$ \\
\hline PA033Q08TA & $\begin{array}{l}\text { Je trouve que la <science au sens large> m'aide } \\
\text { à comprendre les choses qui m'entourent. }\end{array}$ & $\square_{1}$ & $\square_{2}$ & $\square_{3}$ & $\square_{4}$ \\
\hline PA033Q09TA & $\begin{array}{l}\text { En général, les avancées de la <science au sens } \\
\text { large> sont porteuses de progrès sociaux. }\end{array}$ & $\square_{1}$ & $\square_{2}$ & $\square_{3}$ & $\square_{4}$ \\
\hline
\end{tabular}

Considérez-vous que les problèmes environnementaux suivants constituent un grave sujet d'inquiétude pour vous-même et/ou pour d'autres?

(Cochez une case par ligne.)

\begin{tabular}{l|l|c|c|c|c} 
& \multicolumn{1}{|c|}{} & $\begin{array}{c}\text { C'est un } \\
\text { grave sujet } \\
\text { d'inquiétude } \\
\text { pour moi-même } \\
\text { ainsi que } \\
\text { pour d'autres }\end{array}$ & $\begin{array}{c}\text { C'est un } \\
\text { grave sujet } \\
\text { d'inquiétude } \\
\text { pour d'autres gens } \\
\text { de mon pays, } \\
\text { mais pas pour moi }\end{array}$ & $\begin{array}{c}\text { C'est un } \\
\text { grave sujet } \\
\text { d'inquiétude, } \\
\text { mais seulement } \\
\text { dans } \\
\text { d'autres pays }\end{array}$ \\
$\begin{array}{c}\text { Ce n'est un } \\
\text { grave sujet } \\
\text { d'inquiétude } \\
\text { pour personne }\end{array}$ \\
\hline PA035Q01TA & La pollution de l'air & $\square_{1}$ & $\square_{2}$ & $\square_{3}$ & $\square_{4}$ \\
\hline PA035Q03TA & $\begin{array}{l}\text { L'extinction de certaines plantes } \\
\text { et animaux }\end{array}$ & $\square_{1}$ & $\square_{2}$ & $\square_{3}$ & $\square_{4}$ \\
\hline PA035Q04TA & $\begin{array}{l}\text { L'abattage des forêts en vue } \\
\text { de l'exploitation des sols }\end{array}$ & $\square_{1}$ & $\square_{2}$ & $\square_{3}$ & $\square_{4}$ \\
\hline PA035Q05TA & Les pénuries d'eau & $\square_{1}$ & $\square_{2}$ & $\square_{3}$ & $\square_{4}$ \\
\hline PA035Q06TA & Les déchets nucléaires & $\square_{1}$ & $\square_{2}$ & $\square_{3}$ & $\square_{4}$ \\
\hline PA035Q07NA & Les conditions météorologiques extrêmes & $\square_{1}$ & $\square_{2}$ & $\square_{3}$ & $\square_{4}$ \\
\hline PA035Q08NA & $\begin{array}{l}\text { La transmission à l'homme de maladies } \\
\text { d'origine animale }\end{array}$ & $\square_{1}$ & $\square_{2}$ & $\square_{3}$ & $\square_{4}$ \\
\hline
\end{tabular}

\begin{tabular}{|c|c|c|c|c|}
\hline & & $\begin{array}{l}\text { Ils vont } \\
\text { s'atténuer }\end{array}$ & $\begin{array}{c}\text { Ils vont } \\
\text { rester } \\
\text { à peu près } \\
\text { les mêmes }\end{array}$ & $\begin{array}{c}\text { Ils vont } \\
\text { s'aggraver }\end{array}$ \\
\hline PA036Q01TA & La pollution de l'air & $\square_{1}$ & $\square_{2}$ & $\square_{3}$ \\
\hline PA036Q03TA & L'extinction de certaines plantes et animaux & $\square_{1}$ & $\square_{2}$ & $\square_{3}$ \\
\hline PA036Q04TA & L'abattage des forêts en vue de l'exploitation des sols & $\square_{1}$ & $\square_{2}$ & $\square_{3}$ \\
\hline PA036Q05TA & Les pénuries d'eau & $\square_{1}$ & $\square_{2}$ & $\square_{3}$ \\
\hline PA036Q06TA & Les déchets nucléaires & $\square_{1}$ & $\square_{2}$ & $\square_{3}$ \\
\hline PA036Q07NA & Les conditions météorologiques extrêmes & $\square_{1}$ & $\square_{2}$ & $\square_{3}$ \\
\hline PA036Q08NA & La transmission à l'homme de maladies d'origine animale & $\square_{1}$ & $\square_{2}$ & $\square_{3}$ \\
\hline
\end{tabular}




\section{SECTION E VOTRE SITUATION}

PA039

Dans quel pays chacun des membres suivants de la famille de l'enfant est-il né ?

(Cochez une case par colonne.)

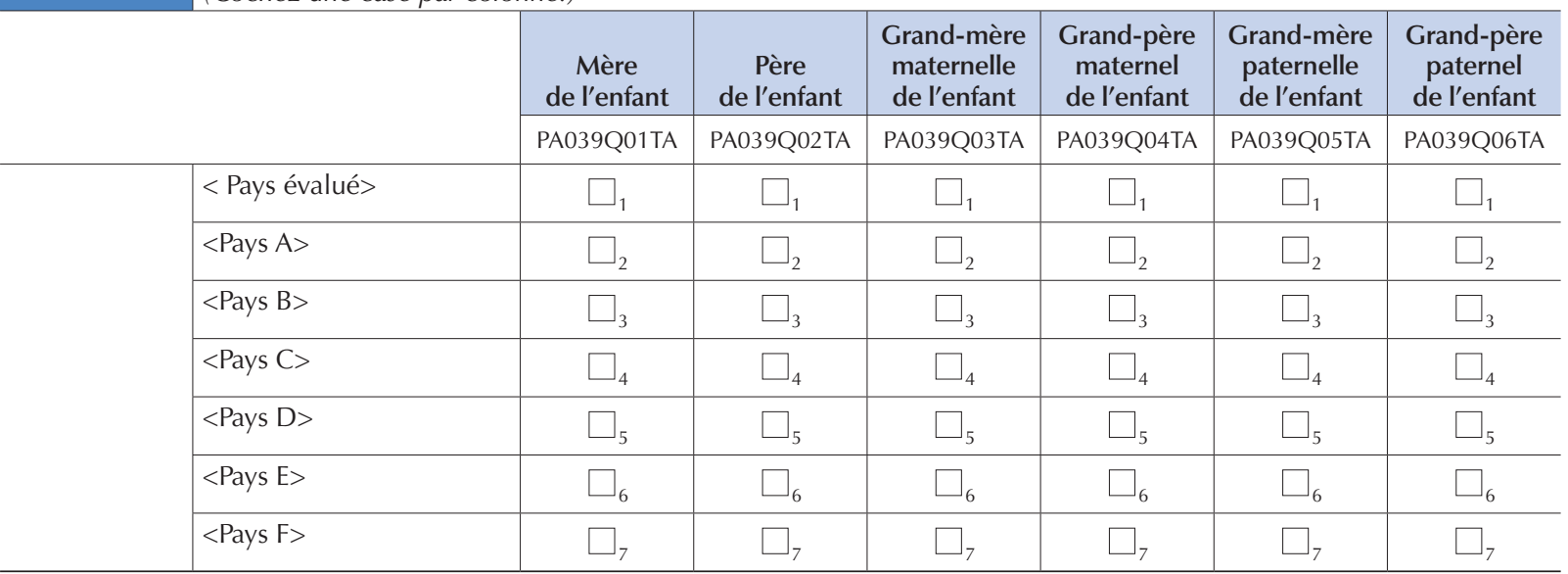

PA041

PA041Q01TA

Veuillez répondre à la question suivante en tenant compte uniquement des dépenses relatives à <l'élève qui vous a remis ce questionnaires.

Au cours des 12 mois écoulés, à peu près combien d'argent avez-vous dépensé pour des services liés à la scolarité de votre enfant?

Pour déterminer ce montant, veuillez tenir compte de tous les frais de scolarité que vous avez réglés à l'établissement de votre enfant, en y ajoutant les honoraires payés pour des cours particuliers éventuellement donnés à votre enfant par des professeurs de l'établissement ou par tout autre professeur, ainsi que les montants versés pour tout cours éventuel de bachotage.

Ne tenez pas compte des dépenses engagées pour l'achat de matériel (par ex., équipements de sport, uniforme de l'école, ordinateur ou manuels scolaires) si vous avez effectué ces achats séparément et que leur prix n'est pas inclus dans le montant global payé à l'établissement.

(Ne cochez qu'une seule case.)

\begin{tabular}{ll|l}
\hline & Rien & $\square_{1}$ \\
\hline & $<$ Plus de 0 euro, mais moins de W euros $>$ & $\square_{2}$ \\
\hline & $<$ W euros ou plus, mais moins de X euros $>$ & $\square_{3}$ \\
\hline & $<$ X euros ou plus, mais moins de Y euros $>$ & $\square_{4}$ \\
\hline & $<$ Y euros ou plus, mais moins de Z euros $>$ & $\square_{5}$ \\
\hline & $<$ Z euros $>$ ou plus & $\square_{6}$ \\
\hline
\end{tabular}

PA042

PA042Q01TA
Quel est le revenu annuel de votre ménage ?

Additionnez le revenu global avant impôts de tous les membres de votre ménage.

Souvenez-vous que nous vous demandons de répondre à ces questions seulement si cela ne vous embarrasse pas de le faire et que toutes vos réponses seront gardées strictement confidentielles.

(Ne cochez qu'une seule case.)

\begin{tabular}{l|l|c} 
& Moins de $<$ A euros $>$ & $\square_{1}$ \\
\hline & $<$ A euros $>$ ou plus, mais moins de $<$ B euros $>$ & $\square_{2}$ \\
\hline & $<$ B euros $>$ ou plus, mais moins de $<$ C euros $>$ & $\square_{3}$ \\
\hline & $<$ C euros $>$ ou plus, mais moins de $<$ D euros $>$ & $\square$ \\
\hline & $<$ D euros $>$ ou plus, mais moins de $<$ E euros $>$ & $\square_{4}$ \\
\hline & $<$ E euros $>$ ou plus & $\square_{5}$ \\
\hline
\end{tabular}




\section{QUESTIONNAIRES ENSEIGNANT(E)S}

(Option internationale)

\section{Versions sources pour la campagne définitive}

Lors de l'enquête PISA 2015, les pays avaient la possibilité d'administrer un questionnaire supplémentaire à l'intention des enseignants. Deux versions de ce questionnaire ont été préparées : la première pour les enseignants de sciences ; et la seconde pour les enseignants d'autres matières. Environ 30 minutes sont nécessaires pour remplir chacune de ces versions.

Le questionnaire à l'intention des enseignants de sciences est destiné à recueillir des renseignements :

- généraux

- sur la formation initiale de l'enseignant et sa formation continue

- sur l'établissement de l'enseignant

- sur les pratiques pédagogiques en sciences

Le questionnaire à l'intention des enseignants d'autres matières est quant à lui destiné à recueillir des renseignements :

- généraux

- sur la formation initiale de l'enseignant et sa formation continue

- sur l'établissement de l'enseignant

- sur les pratiques pédagogiques

\section{PARTIE A QUESTIONNAIRE À L'INTENTION DES ENSEIGNANT(E)S DE SCIENCES}

\section{Renseignements généraux}

TC001 Êtes-vous une femme ou un homme?

TC001Q01NA (Sélectionnez une réponse.)

\begin{tabular}{|l|c}
\hline Une femme & $\square_{1}$ \\
\hline Un homme & $\square_{2}$
\end{tabular}

$\mathrm{TCOO2}$

TC002Q01NA
Quel âge avez-vous?

(Positionnez le curseur sur le nombre d'années correspondant.)

Nombre d'années:

Curseur : position initiale ; plage : « 20 ans ou moins » - 70 ans ou plus » ; graduation=1.

$\mathrm{TCOO4}$

TC004Q01NA
Sous quel contrat de travail enseignez-vous dans cet établissement ?

(Sélectionnez une réponse.)

\begin{tabular}{|l|c}
\hline Contrat de travail permanent (à durée indéterminée sans expiration prévue avant l'âge de la retraite) & $\square_{1}$ \\
\hline Contrat de travail à durée déterminée de plus d'une année scolaire & $\square_{2}$ \\
\hline Contrat de travail à durée déterminée d'une année scolaire ou moins & $\square_{3}$ \\
\hline
\end{tabular}


(Tenez compte de tous vos postes actuels d'enseignant(e) pour déterminer si vous enseignez à temps plein ou à temps partiel.)

(Sélectionnez une réponse par ligne.)

\begin{tabular}{|c|c|c|c|c|c|}
\hline & & $\begin{array}{c}\text { À temps plein } \\
\text { (plus de } 90 \% \\
\text { du temps } \\
\text { de travail à } \\
\text { temps plein) }\end{array}$ & $\begin{array}{c}\text { À temps } \\
\text { partiel (entre } \\
71 \text { et } 90 \% \\
\text { du temps } \\
\text { de travail à } \\
\text { temps plein) }\end{array}$ & $\begin{array}{c}\text { À temps } \\
\text { partiel (entre } \\
50 \text { et } 70 \% \\
\text { du temps } \\
\text { de travail à } \\
\text { temps plein) }\end{array}$ & $\begin{array}{c}\text { À temps } \\
\text { partiel (moins } \\
\text { de } 50 \% \\
\text { du temps } \\
\text { de travail à } \\
\text { temps plein) }\end{array}$ \\
\hline TC005Q01NA & Mon poste dans cet établissement & $\square_{1}$ & $\square_{2}$ & $\square_{3}$ & $\square_{4}$ \\
\hline TC005Q02NA & L'ensemble de mes postes d'enseignant(e) & $\square_{1}$ & $\square_{2}$ & $\square_{3}$ & $\square_{4}$ \\
\hline
\end{tabular}

TC006

TC006Q01NA
Dans combien d'établissements avez-vous travaillé au cours de votre carrière d'enseignant(e) ?

(Prenez en compte tous les établissements, même si vous avez travaillé dans plusieurs d'entre eux simultanément.)

(Positionnez le curseur sur le nombre correspondant d'établissements.)

Nombre d'établissements :

Curseur : position initiale ; plage : « 1 établissement » - « 20 établissements ou plus » ; graduation=1

\begin{tabular}{l|l|l}
\multicolumn{1}{c|}{$\begin{array}{l}\text { Combien d'années d'expérience professionnelle avez-vous ? } \\
\text { (Arrondissez les nombres d'années à l'unité, que vous ayez travaillé à temps partiel ou à temps plein. Positionnez le curseur } \\
\text { sur le nombre d'années correspondant. Indiquez " } 0 \text { » [zéro] si une des options ne correspond pas à votre situation.) }\end{array}$} \\
\hline TC007Q01NA & Année(s) d'expérience en tant qu'enseignant(e) dans cet établissement \\
\hline TC007Q02NA & Année(s) d'expérience en tant qu'enseignant(e) en tout & \\
\hline $\begin{array}{l}\text { Curseur : position initiale ; plage : « } 0 \text { année » - " } 50 \text { années ou plus »; graduation=1. } \\
\text { Contrôle de cohérence/rappel si la réponse à la question TC007Q01NA est supérieure à celle donnée à la question TC007Q02NA. }\end{array}$
\end{tabular}

\section{Votre formation initiale et votre formation continue}

TC012
TC013

TC013Q01NA
Quel est le niveau du diplôme le plus élevé que vous ayez obtenu dans l'enseignement institutionnel ?

(Sélectionnez une réponse.)

\begin{tabular}{|l|c|}
\hline <Inférieur au Niveau 5 de la CITE $>$ & $\square_{1}$ \\
\hline$<$ Niveau 5B de la CITE $>$ & $\square_{2}$ \\
\hline$<$ Niveau 5A de la CITE - Bachelier / licence> & $\square_{3}$ \\
\hline$<$ Niveau 5A de la CITE - Master $>$ & $\square_{4}$ \\
\hline$<$ Niveau 6 de la CITE $>$ & $\square_{5}$
\end{tabular}

À la fin du <Niveau 3 de la CITE ou inférieur>, aviez-vous pour objectif de poursuivre une carrière dans l'enseignement?

(Sélectionnez une réponse.)

\begin{tabular}{l|l|l} 
& Oui & $\square$ \\
\hline & Non & $\square_{1}$ \\
\hline
\end{tabular}

Avez-vous terminé une formation ou des études d'enseignant ?

(Sélectionnez une réponse.)

\begin{tabular}{|l|l}
\hline Oui & $\square_{1}$ \\
\hline Non & $\square_{2}$ \\
\hline
\end{tabular}


TC015

TC015Q01NA
Comment avez-vous obtenu les qualifications requises pour enseigner?

(Sélectionnez une réponse.)

J'ai suivi uneformation ou des études classiques d'enseignant en fréquentant un <institut de formation

pédagogique habilité à former des enseignants>.

J'ai suivi uneformation ou des études d'enseignantdans le cadre de la formation continue.

J'ai suivi uneformation ou des études d'enseignant sur le lieu de travail.

J'ai suivi une formation dans une autre profession à caractère pédagogique.

Autre

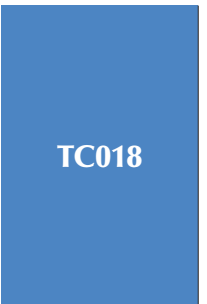

Les groupes de matières suivants figuraient-ils au programme de votre formation d'enseignant ou d'une autre qualification professionnelle? Enseignez-vous ces matières en <grade modal du pays pour les élèves de $\mathbf{1 5}$ ans> au cours de cette année scolaire?

(Comme il s'agit d'une enquête internationale, nous avons dû choisir des catégories assez vastes et y classer de nombreuses matières enseignées à l'école. Si l'intitulé exact d'une de vos matières ne figure pas dans la liste, sélectionnez la catégorie qui vous semble le mieux correspondre à cette matière.)

(Si vous avez besoin d'explications au sujet des termes utilisés dans cette question, utilisez le bouton d'aide.) (Sélectionnez toutes les réponses qui conviennent.)

\begin{tabular}{|c|c|c|c|}
\hline & & professionnelle & de cette année scolaire \\
\hline TC018Q01N & Lecture, expression écrite et littérature & $\square_{1}$ & $\square_{1}$ \\
\hline TC018Q02N & Mathématiques & $\square_{1}$ & $\square_{1}$ \\
\hline TC018Q03N & Sciences & $\square_{1}$ & $\square_{1}$ \\
\hline TC018Q04N & Technologie & $\square_{1}$ & $\square_{1}$ \\
\hline TC018Q05N & Sciences humaines & $\square_{1}$ & $\square_{1}$ \\
\hline TC018Q06N & Langues vivantes étrangères & $\square_{1}$ & $\square_{1}$ \\
\hline TC018Q07N & Langues anciennes (par ex. le latin) & $\square_{1}$ & $\square_{1}$ \\
\hline TC018Q08N & Disciplines artistiques & $\square_{1}$ & $\square_{1}$ \\
\hline TC018Q09N & Éducation physique & $\square_{1}$ & $\square_{1}$ \\
\hline TC018Q10N & Religion et/ou morale & $\square_{1}$ & $\square_{1}$ \\
\hline TC018Q11N & Disciplines pratiques et professionnelles & $\square_{1}$ & $\square_{1}$ \\
\hline
\end{tabular}

Bouton d'aide Lecture, expression écrite et littérature : la lecture et l'expression écrite (et la littérature) dans la langue maternelle, dans la langue d'enseignement et dans la langue nationale (ou régionale) en tant que deuxième langue (pour les allophones), la linguistique, l'expression orale et la littérature

Mathématiques : les mathématiques, les statistiques, la géométrie, l'algèbre, etc.

Sciences : les sciences naturelles, la physique, la chimie, la biologie, la biologie humaine, les sciences de la Terre et de l'Univers, les sciences de l'environnement, ainsi que l'agronomie, I'horticulture et la foresterie

Technologie : l'initiation à la technologie, dont les technologies de l'information, l'informatique, la construction et la topométrie, l'électronique, le graphisme et le design, la dactylographie, le traitement de texte, les techniques $d^{\prime}$ atelier et le dessin technique

Sciences humaines : les sciences humaines, l'étude des collectivités, l'étude des sociétés contemporaines, l'économie, l'étude du milieu, la géographie, I'histoire, les sciences sociales, le droit, l'étude du pays, la pensée éthique et la philosophie

Langues vivantes étrangères : les langues vivantes autres que la langue d'enseignement

Langues anciennes (par ex. le latin)

Disciplines artistiques : les arts, la musique, les arts visuels, les arts appliqués, le théâtre, les arts du spectacle,

la photographie, le dessin, I'artisanat et la couture artistique

Éducation physique : l'éducation physique, la gymnastique, la danse et l'hygiène

Religion et/ou morale : la religion, l'histoire des religions, la culture religieuse et la morale

Disciplines pratiques et professionnelles : les enseignements à vocation professionnelle (qui préparent à exercer un métier spécifique), la technique, les arts ménagers, la comptabilité, le commerce, l'enseignement professionnel, I'habillement et la couture, les cours de conduite, l'économie ménagère, les cours polytechniques, le secrétariat, le tourisme et l'accueil et l'artisanat. 


\begin{tabular}{c|l|l|l} 
& $\begin{array}{l}\text { Quelle proportion de votre formation d'enseignant ou d'une autre qualification professionnelle } \\
\text { était consacrée à chacun des domaines suivants ? } \\
\text { TC029 }\end{array}$ & $\begin{array}{l}\text { (Pour chaque domaine, indiquez un pourcentage approximatif, par exemple, " } 20 \text { » à la première ligne } \\
\text { pour indiquer que } 20 \% \text { du temps de votre formation initiale étaient consacrés à un contenu en rapport } \\
\text { avec les <sciences> et la technologie.) } \\
\text { (La somme des pourcentages doit être égale à 100.) }\end{array}$ & - \\
\hline TC029Q01NA & $\begin{array}{l}\text { Contenus scientifiques et technologiques : connaissances et compétences dans n'importe quelle } \\
\text { discipline en rapport avec les <sciences> }\end{array}$ & \\
\hline TC029Q02NA & $\begin{array}{l}\text { Enseignement et apprentissage des <cours de sciences> : méthodologie d'enseignement } \\
\text { des <cours de sciences>, compétences pédagogiques (par ex. le recours à des expériences), } \\
\text { conceptions erronées des élèves }\end{array}$ & - \\
\hline TC029Q03NA & $\begin{array}{l}\text { Sujets pédagogiques généraux : par ex., les interactions élève-enseignant, la gestion des classes, } \\
\text { l'évaluation des élèves, l'enseignement aux élèves présentant des besoins éducatifs particuliers }\end{array}$ & - \\
\hline TC029Q04NA & Autres sujets & $\%$ \\
\hline Contrôle de cohérence/rappel si la somme est supérieure ou inférieure à $100 \%$.
\end{tabular}

Quelle proportion de votre formation d'enseignant ou d'une autre qualification professionnelle était consacrée à chacun des domaines suivants ?

(Pour chaque domaine, indiquez un pourcentage approximatif, par exemple, "20 » à la première ligne pour indiquer que 20 \% du temps de votre formation initiale étaient consacrés à un contenu en rapport avec les <sciences> et la technologie.)

(La somme des pourcentages doit être égale à 100.)

Contenus scientifiques et technologiques : connaissances et compétences dans n'importe quelle discipline en rapport avec les <sciences $>$

Enseignement et apprentissage des <cours de sciences $>$ : méthodologie d'enseignement des <cours de sciences $>$, compétences pédagogiques (par ex., le recours à des expériences), conceptions erronées des élèves

Sujets pédagogiques généraux : par ex. les interactions élève-enseignant, la gestion des classes, l'évaluation des élèves, I'enseignement aux élèves présentant des besoins éducatifs particuliers

Autres sujets

$<<$ Précendent

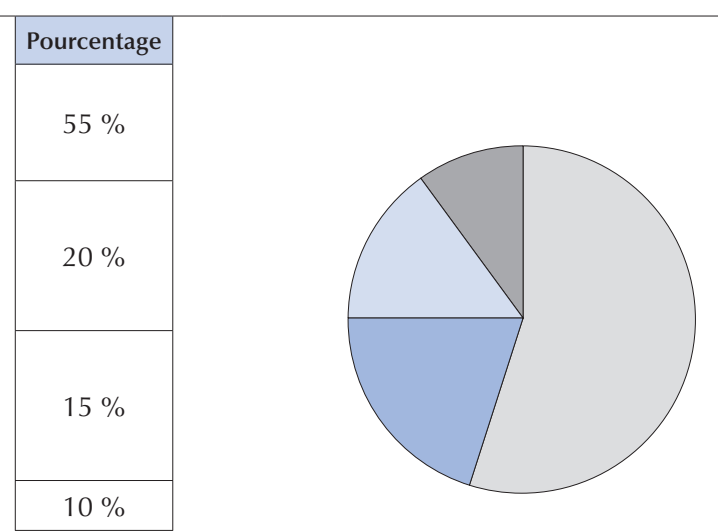

$10 \%$

Le diagramme circulaire offre un feedback interactif immédiat et le répondant peut changer ses réponses autant de fois qu'il le souhaite.

TC020

Au cours des 12 derniers mois, avez-vous participé aux activités suivantes ?

(Sélectionnez une réponse par ligne.)

\begin{tabular}{l|l|c|c}
\multicolumn{2}{c|}{} & Oui & Non \\
\hline TC020Q01NA & À un programme de qualification (par ex. <sanctionné par un diplôme>) & $\square_{1}$ & $\square_{2}$ \\
\hline TC020Q02NA & $\begin{array}{l}\text { Aux activités d'un réseau d'enseignants axé sur la formation continue } \\
\text { des enseignants }\end{array}$ & $\square_{1}$ & $\square_{2}$ \\
\hline TC020Q03NA & $\begin{array}{l}\text { À des recherches individuelles ou en groupe sur un sujet en rapport avec } \\
\text { votre profession }\end{array}$ & $\square_{1}$ & $\square_{2}$ \\
\hline TC020Q04NA & $\begin{array}{l}\text { À des activités de tutorat, d'observation collégiale ou de coaching officiellement } \\
\text { organisées dans votre établissement }\end{array}$ & $\square_{1}$ & $\square_{2}$ \\
\hline TC020Q05NA & $\begin{array}{l}\text { Lecture d'ouvrages spécialisés (par ex. des revues, des travaux de recherche, } \\
\text { des thèses). }\end{array}$ & $\square_{1}$ & $\square_{2}$ \\
\hline TC020Q06NA & Dialogue informel avec vos collègues sur l'amélioration de votre enseignement & $\square_{1}$ & $\square_{2}$ \\
\hline
\end{tabular}




\begin{tabular}{|c|c|c|}
\hline TC030 & \multicolumn{2}{|l|}{$\begin{array}{l}\text { Au cours des } \mathbf{1 2} \text { derniers mois, quelle proportion de vos activités de formation continue } \\
\text { a été consacrée à chacun des domaines suivants ? } \\
\text { (Pour chaque domaine, indiquez un pourcentage approximatif, par exemple, " } 20 \text { » à la première ligne } \\
\text { pour indiquer que } 20 \% \text { du temps de votre formation initiale étaient consacrés à un contenu en rapport } \\
\text { avec les <sciences> et la technologie.) } \\
\text { (La somme des pourcentages doit être égale à 100.) }\end{array}$} \\
\hline TC030Q01NA & $\begin{array}{l}\text { Contenus scientifiques et technologiques : connaissances et compétences dans n'importe quelle } \\
\text { discipline en rapport avec les <sciences }>\end{array}$ & — $\%$ \\
\hline TC030Q02NA & $\begin{array}{l}\text { Enseignement et apprentissage des <cours de sciences }>\text { : méthodologie } \mathrm{d}^{\prime} \text { enseignement des }<\text { cours } \\
\text { de sciences }>\text {, compétences pédagogiques (par ex. le recours à des expériences), conceptions } \\
\text { erronées des élèves }\end{array}$ & — $\%$ \\
\hline TC030Q03NA & $\begin{array}{l}\text { Sujets pédagogiques généraux : par ex. les interactions élève-enseignant, la gestion des classes, } \\
\text { l'évaluation des élèves, I'enseignement aux élèves présentant des besoins éducatifs particuliers }\end{array}$ & — \\
\hline TC030Q04NA & Autres sujets & $\%$ \\
\hline
\end{tabular}

Au cours des 12 derniers mois, quelle proportion de vos activités de formation continue a été consacrée à chacun des domaines suivants ?

(Pour chaque domaine, indiquez un pourcentage approximatif, par exemple, "20 » à la première ligne pour indiquer que 20 \% du temps de votre formation initiale étaient consacrés à un contenu en rapport avec les <sciences> et la technologie.)

(La somme des pourcentages doit être égale à 100.)

Contenus scientifiques et technologiques : connaissances et compétences dans n'importe quelle discipline en rapport avec les $<$ sciences $>$

Enseignement et apprentissage des <cours de sciences $>$ : méthodologie d'enseignement des <cours de sciences $>$, compétences pédagogiques (par ex. le recours à des expériences), conceptions erronées des élèves

Sujets pédagogiques généraux : par ex. les interactions élève-enseignant, la gestion des classes, l'évaluation des élèves, I'enseignement aux élèves présentant des besoins éducatifs particuliers

Autres sujets
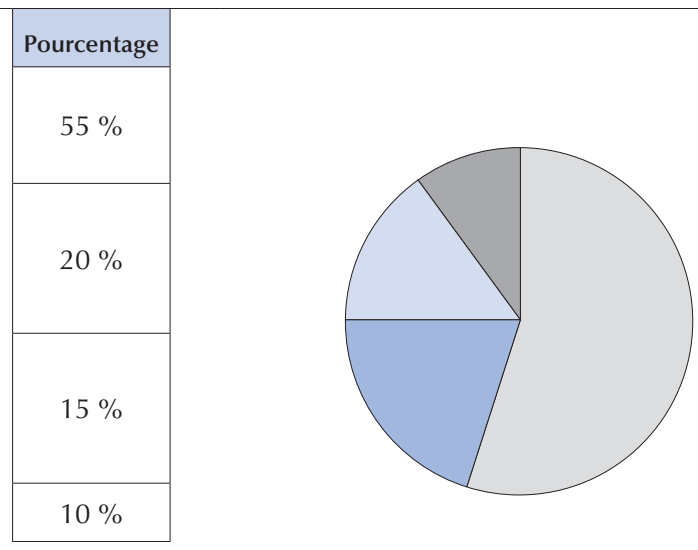

$$
<<\text { Précédent }
$$

Suivant $>>$

Le diagramme circulaire offre un feedback interactif immédiat et le répondant peut changer ses réponses autant de fois qu'il le souhaite.

TC021

\begin{tabular}{|l|c}
\hline Oui & $\square_{1}$ \\
\hline Non & $\square_{2}$ \\
\hline
\end{tabular}




\section{Votre établissement}

TC028

L'enseignement que votre établissement est à même de dispenser est-il affecté par les problèmes suivants?

(Sélectionnez une réponse par ligne.)

\begin{tabular}{|c|c|c|c|c|c|}
\hline & & Pas du tout & Très peu & $\begin{array}{l}\text { Dans } \\
\text { une certaine } \\
\text { mesure }\end{array}$ & Beaucoup \\
\hline TC028Q01NA & Manque de personnel enseignant & $\square_{1}$ & $\square_{2}$ & $\square_{3}$ & $\square_{4}$ \\
\hline TC028Q02NA & Personnel enseignant inadéquat ou peu qualifié & $\square_{1}$ & $\square_{2}$ & $\square_{3}$ & $\square_{4}$ \\
\hline TC028Q03NA & Manque de personnel auxiliaire & $\square_{1}$ & $\square_{2}$ & $\square_{3}$ & $\square_{4}$ \\
\hline TC028Q04NA & Personnel auxiliaire inadéquat ou peu qualifié & $\square_{1}$ & $\square_{2}$ & $\square_{3}$ & $\square_{4}$ \\
\hline TC028Q05NA & $\begin{array}{l}\text { Manque de matériel pédagogique (par ex. manuels } \\
\text { scolaires, équipement informatique, matériel } \\
\text { de bibliothèque ou de laboratoire) }\end{array}$ & $\square_{1}$ & $\square_{2}$ & $\square_{3}$ & $\square_{4}$ \\
\hline TC028Q06NA & $\begin{array}{l}\text { Matériel pédagogique inadéquat ou de mauvaise qualité } \\
\text { (par ex. manuels scolaires, équipement informatique, } \\
\text { matériel de bibliothèque ou de laboratoire) }\end{array}$ & $\square_{1}$ & $\square_{2}$ & $\square_{3}$ & $\square_{4}$ \\
\hline TC028Q07NA & $\begin{array}{l}\text { Manque d'infrastructures (par ex. bâtiments, terrain, } \\
\text { chauffage/climatisation, systèmes d'éclairage et } \\
\text { acoustiques) }\end{array}$ & $\square_{1}$ & $\square_{2}$ & $\square_{3}$ & $\square_{4}$ \\
\hline TC028Q08NA & $\begin{array}{l}\text { Infrastructures inadéquates ou de mauvaise qualité } \\
\text { (par ex. bâtiments, terrain, chauffage/ climatisation, } \\
\text { systèmes d'éclairage et acoustiques) }\end{array}$ & $\square_{1}$ & $\square_{2}$ & $\square_{3}$ & $\square_{4}$ \\
\hline
\end{tabular}

TC039

TC039Q01NA

Existe-t-il un programme officiel pour les <cours de sciences $>$ destiné au < grade modal du pays pour les élèves de 15 ans>?

(Tenez compte des politiques à l'échelle nationale, provinciale, régionale ou de l'établissement.)

(Sélectionnez une réponse.)

\begin{tabular}{|l|l}
\hline Oui & $\square_{1}$ \\
\hline Non & $\square_{2}$ \\
\hline
\end{tabular}

$\rightarrow$ Ne répondre à cette question que si TC039 = « Oui ». Sinon, passer à la question TC031.

Considérez le programme officiel pour les <cours de sciences $>$ destiné au < grade modal du pays pour les élèves de 15 ans>. Quelle importance y accorde-t-on aux approches et procédés suivants ?

(Sélectionnez une réponse par ligne.)

\begin{tabular}{l|l|c|c|c|c}
\multicolumn{2}{l|}{} & $\begin{array}{c}\text { Aucune } \\
\text { importance }\end{array}$ & $\begin{array}{c}\text { Très peu } \\
\text { d'importance }\end{array}$ & $\begin{array}{c}\text { Quelque } \\
\text { importance }\end{array}$ & $\begin{array}{c}\text { Beaucoup } \\
\text { d'importance }\end{array}$ \\
\hline TC041Q01NA & $\begin{array}{l}\text { Connaître des faits et principes scientifiques } \\
\text { fondamentaux }\end{array}$ & $\square_{1}$ & $\square_{2}$ & $\square_{3}$ & $\square_{4}$ \\
\hline TC041Q02NA & Observer les phénomènes naturels et les décrire & $\square_{1}$ & $\square_{2}$ & $\square_{3}$ & $\square_{4}$ \\
\hline TC041Q03NA & Expliquer le contenu de la leçon & $\square_{1}$ & $\square_{2}$ & $\square_{3}$ & $\square_{4}$ \\
\hline TC041Q04NA & Concevoir et organiser des expériences ou des recherches & $\square_{1}$ & $\square_{2}$ & $\square_{3}$ & $\square_{4}$ \\
\hline TC041Q05NA & Mener des expériences ou des recherches & $\square_{1}$ & $\square_{2}$ & $\square_{3}$ & $\square_{4}$ \\
\hline TC041Q06NA & Intégrer les sciences à d'autres disciplines & $\square_{1}$ & $\square_{2}$ & $\square_{3}$ & $\square_{4}$ \\
\hline TC041Q07NA & $\begin{array}{l}\text { Établir un lien entre ce que les élèves apprennent } \\
\text { et leur vie quotidienne }\end{array}$ & $\square_{1}$ & $\square_{2}$ & $\square_{3}$ & $\square_{4}$ \\
\hline TC041Q08NA & Intégrer le vécu dedifférents groupes ethniques/ culturels & $\square_{1}$ & $\square_{2}$ & $\square_{3}$ & $\square_{4}$ \\
\hline
\end{tabular}


TC043

TC043Q01NA

$\rightarrow$ Ne répondre à cette question que si TC039 = «Oui ». Sinon, passer à la question TC031.

Les parents sont-ils informés de l'existence et du contenu du programme officiel pour les <cours de sciences> (par ex. lors d'une réunion parents/professeurs ou par une lettre d'information)?

(Sélectionnez une réponse.)

\begin{tabular}{l|l}
\hline Oui & $\square_{1}$ \\
\hline Non & $\square_{2}$ \\
\hline
\end{tabular}

Dans quelle mesure êtes-vous d'accord ou non avec les affirmations suivantes concernant votre coopération habituelle avec vos collègues enseignants de <cours de sciences> ?

(Sélectionnez une réponse par ligne.)

\begin{tabular}{|c|c|c|c|c|c|}
\hline & & $\begin{array}{l}\text { Pas du tout } \\
\text { d'accord }\end{array}$ & $\begin{array}{c}\text { Pas } \\
\text { d'accord }\end{array}$ & D'accord & $\begin{array}{l}\text { Tout à fait } \\
\text { d'accord }\end{array}$ \\
\hline TC031Q04NA & $\begin{array}{l}\text { Nous discutons des critères de réussite en <cours } \\
\text { de sciences }>\text { quand nous préparons les évaluations. }\end{array}$ & & & & \\
\hline TC031Q07NA & $\begin{array}{l}\text { Il nous semble naturel de coopérer pour déterminer quels } \\
\text { devoirs donner à nos élèves. }\end{array}$ & & & & \\
\hline TC031Q11NA & $\begin{array}{l}\text { Nous discutons des critères que nous utilisons pour noter } \\
\text { les évaluations écrites. }\end{array}$ & & & & \\
\hline TC031Q13NA & $\begin{array}{l}\text { Nous échangeons des exercices de différents niveaux } \\
\text { de difficulté à faire en classe ou à la maison. }\end{array}$ & & & & \\
\hline TC031Q14NA & $\begin{array}{l}\text { Je prépare une partie de mes cours avec mes collègues } \\
\text { enseignants de }<\text { cours de sciences }>\text {. }\end{array}$ & & & & \\
\hline TC031Q15NA & $\begin{array}{l}\text { Nous discutons des façons d'enseigner des stratégies } \\
\text { et des techniques d'apprentissage à nos élèves. }\end{array}$ & & & & \\
\hline TC031Q18NA & $\begin{array}{l}\text { Mes compétences et intérêts personnels profitent } \\
\text { à mes collègues enseignants de <cours de sciences }>\text {. }\end{array}$ & $\square_{1}$ & $\square_{2}$ & & \\
\hline TC031Q20NA & $\begin{array}{l}\text { Nous discutons des moyens nous permettant de mieux } \\
\text { identifier les points forts et les points faibles de chaque élève. }\end{array}$ & $\square_{1}$ & $\square_{2}$ & $\square$ & $\square_{4}$ \\
\hline
\end{tabular}

Nous aimerions savoir quels sentiments vos fonctions vous inspirent dans l'ensemble. Dans quelle mesure êtes-vous d'accord avec les affirmations suivantes?

(Sélectionnez une réponse par ligne.)

\begin{tabular}{l|l|c|c|c|c}
\multicolumn{1}{l|}{} & \multicolumn{1}{c|}{$\begin{array}{c}\text { Pas du tout } \\
\text { d'accord }\end{array}$} & $\begin{array}{c}\text { Tout à fait } \\
\text { d'accord }\end{array}$ & $\begin{array}{c}\text { D'accord } \\
\text { d'accord }\end{array}$ \\
\hline TC026Q01NA & $\begin{array}{l}\text { Les avantages du métier d'enseignant compensent } \\
\text { largement ses inconvénients. }\end{array}$ & $\square_{1}$ & $\square_{2}$ & $\square_{3}$ & $\square_{4}$ \\
\hline TC026Q02NA & $\begin{array}{l}\text { Si c'était à refaire, je choisirais de nouveau le métier } \\
\text { d'enseignant(e). }\end{array}$ & $\square_{1}$ & $\square_{2}$ & $\square_{3}$ & $\square_{4}$ \\
\hline TC026Q04NA & Je regrette ma décision de devenir enseignant(e). & $\square_{1}$ & $\square_{2}$ & $\square_{3}$ & $\square_{4}$ \\
\hline TC026Q05NA & J'aime travailler dans cet établissement. & $\square_{1}$ & $\square_{2}$ & $\square_{3}$ & $\square_{4}$ \\
\hline TC026Q06NA & $\begin{array}{l}\text { Je me demande si je n'aurais pas mieux fait de choisir } \\
\text { une autre profession. }\end{array}$ & $\square_{1}$ & $\square_{2}$ & $\square_{3}$ & $\square_{4}$ \\
\hline TC026Q07NA & $\begin{array}{l}\text { Mon établissement est un endroit agréable où travailler, } \\
\text { je le recommanderais à d'autres enseignants. }\end{array}$ & $\square_{1}$ & $\square_{2}$ & $\square_{3}$ & $\square_{4}$ \\
\hline TC026Q09NA & Je suis satisfait(e) de mon travail dans cet établissement. & $\square_{1}$ & $\square_{2}$ & $\square_{3}$ & $\square_{4}$ \\
\hline TC026Q10NA & Dans l'ensemble, mon travail me donne satisfaction. & $\square_{1}$ & $\square_{2}$ & $\square_{3}$ & $\square$. \\
\hline
\end{tabular}




\section{Les pratiques pédagogiques en sciences}

\section{TC037}

À quelle fréquence les situations suivantes se produisent-elles pendant vos <cours de sciences $>$ ?

(Sélectionnez une réponse par ligne.)

\begin{tabular}{|c|c|c|c|c|c|}
\hline & & $\begin{array}{c}\text { Jamais ou } \\
\text { presque } \\
\text { jamais }\end{array}$ & Parfois & Souvent & $\begin{array}{l}\text { À chaque } \\
\text { cours ou } \\
\text { presque }\end{array}$ \\
\hline TC037Q01NA & $\begin{array}{l}\text { Les élèves sont amenés à tirer des conclusions } \\
\text { d'une expérience qu'ils ont réalisée. }\end{array}$ & $\square_{1}$ & $\square_{2}$ & $\square_{3}$ & $\square_{4}$ \\
\hline TC037Q02NA & Les élèvesont l'occasion d'exposerleurs idées. & $\square_{1}$ & $\square_{2}$ & $\square_{3}$ & $\square_{4}$ \\
\hline TC037Q03NA & J'explique des concepts scientifiques. & $\square_{1}$ & $\square_{2}$ & $\square_{3}$ & $\square_{4}$ \\
\hline TC037Q04NA & Les élèves discutent par petits groupes. & $\square_{1}$ & $\square_{2}$ & $\square_{3}$ & $\square_{4}$ \\
\hline TC037Q05NA & $\begin{array}{l}\text { Une discussion a lieu à l'échelle de la classe, } \\
\text { et j'y participe. }\end{array}$ & $\square_{1}$ & $\square_{2}$ & $\square_{3}$ & $\square_{4}$ \\
\hline TC037Q06NA & Nous discutons de questions scientifiques actuelles. & $\square_{1}$ & $\square_{2}$ & $\square_{3}$ & $\square_{4}$ \\
\hline TC037Q07NA & Les élèves font descalculs à l'aidede formules scientifiques. & $\square_{1}$ & $\square_{2}$ & $\square_{3}$ & $\square_{4}$ \\
\hline TC037Q08NA & J'utiliseun tableau blanc interactif. & $\square_{1}$ & $\square_{2}$ & $\square_{3}$ & $\square_{4}$ \\
\hline TC037Q09NA & $\begin{array}{l}\text { Les élèves réalisentleur propre étude scientifique } \\
\text { et la recherche associée. }\end{array}$ & $\square_{1}$ & $\square_{2}$ & $\square_{3}$ & $\square_{4}$ \\
\hline TC037Q10NA & Je discute des questions posées par les élèves. & $\square_{1}$ & $\square_{2}$ & $\square_{3}$ & $\square_{4}$ \\
\hline TC037Q11NA & Les élèves font des travaux pratiques. & $\square_{1}$ & $\square_{2}$ & $\square_{3}$ & $\square_{4}$ \\
\hline TC037Q12NA & $\begin{array}{l}\text { Les élèves rédigent des comptes rendus d'expériences } \\
\text { en laboratoire. }\end{array}$ & $\square_{1}$ & $\square_{2}$ & $\square_{3}$ & $\square_{4}$ \\
\hline TC037Q13NA & Je démontre une idée. & $\square_{1}$ & $\square_{2}$ & $\square_{3}$ & $\square_{4}$ \\
\hline TC037Q14NA & Je discute de questions d'ordre pratique. & $\square_{1}$ & $\square_{2}$ & $\square_{3}$ & $\square_{4}$ \\
\hline TC037Q15NA & Les élèves lisent des documents dans un manuel scolaire. & $\square_{1}$ & $\square_{2}$ & $\square_{3}$ & $\square_{4}$ \\
\hline TC037Q16NA & Les élèves notent ce qui est écrit au tableau. & $\square_{1}$ & $\square_{2}$ & $\square_{3}$ & $\square_{4}$ \\
\hline TC037Q17NA & $\begin{array}{l}\text { Les élèves discutent sur la base de documents du manuel } \\
\text { scolaire. }\end{array}$ & $\square_{1}$ & $\square_{2}$ & $\square_{3}$ & $\square_{4}$ \\
\hline TC037Q18NA & Les élèves regardent des films vidéo. & $\square_{1}$ & $\square_{2}$ & $\square_{3}$ & $\square_{4}$ \\
\hline TC037Q19NA & Les élèves utilisent Internet. & $\square_{1}$ & $\square_{2}$ & $\square_{3}$ & $\square_{4}$ \\
\hline TC037Q20NA & La classe corrige les devoirs à la maison ou une évaluation. & $\square_{1}$ & $\square_{2}$ & $\square_{3}$ & $\square_{4}$ \\
\hline TC037Q21NA & Les élèves remplissent des feuilles de calcul. & $\square_{1}$ & $\square_{2}$ & $\square_{3}$ & $\square_{4}$ \\
\hline TC037Q22NA & Les élèves font une présentation au reste de la classe. & $\square_{1}$ & $\square_{2}$ & $\square_{3}$ & $\square_{4}$ \\
\hline
\end{tabular}

$\mathrm{TCO33}$

Dans quelle mesure pouvez-vous (ou pourriez-vous) faire ce qui suit ?

(Sélectionnez une réponse par ligne.)

\begin{tabular}{l|l|c|c|c|c}
\multicolumn{2}{l|}{} & Pas du tout & Très peu & $\begin{array}{c}\text { Dans } \\
\text { une certaine } \\
\text { mesure }\end{array}$ & $\begin{array}{c}\text { Dans } \\
\text { une grande } \\
\text { mesure }\end{array}$ \\
\hline TC033Q04NA & $\begin{array}{l}\text { Concevoir des expériences et des activités pratiques pour } \\
\text { I'<apprentissage fondé sur une démarche de recherche> }\end{array}$ & $\square_{1}$ & $\square_{2}$ & $\square_{3}$ \\
\hline TC033Q05NA & $\begin{array}{l}\text { Donner des exercices personnalisés aux élèves les plus } \\
\text { faibles comme aux plus forts }\end{array}$ & $\square_{1}$ & $\square_{2}$ & $\square_{3}$ \\
\hline TC033Q06NA & Utiliser des modalités d'évaluation variées & $\square_{1}$ & $\square_{2}$ & $\square_{3}$ & $\square_{4}$ \\
\hline TC033Q08NA & $\begin{array}{l}\text { Favoriser les discussions entre élèves sur la façon } \\
\text { d'interpréter les résultats d'une expérience }\end{array}$ & $\square_{1}$ & $\square_{2}$ & $\square_{3}$ & $\square_{4}$ \\
\hline
\end{tabular}


Dans quelle mesure pouvez-vous (ou pourriez-vous) faire ce qui suit ?

(Si vous avez besoin d'explications au sujet du terme "ma discipline scientifique », utilisez le bouton d'aide.) (Sélectionnez une réponse par ligne.)

\begin{tabular}{|c|c|c|c|c|c|}
\hline & & Pas du tout & Très peu & $\begin{array}{l}\text { Dans } \\
\text { une certaine } \\
\text { mesure }\end{array}$ & $\begin{array}{l}\text { Dans } \\
\text { une grande } \\
\text { mesure }\end{array}$ \\
\hline TC034Q01NA & $\begin{array}{l}\text { Expliquer un concept scientifique complexe } \\
\text { à un collègue }\end{array}$ & $\square_{1}$ & $\square_{2}$ & $\square_{3}$ & $\square_{4}$ \\
\hline TC034Q02NA & $\begin{array}{l}\text { Énoncer et défendre une position éclairée sur } \\
\text { des questions éthiques liées aux<sciences }>\end{array}$ & $\square_{1}$ & $\square_{2}$ & $\square_{3}$ & $\square_{4}$ \\
\hline TC034Q04NA & Lire des articles pointus dans ma discipline scientifique & $\square_{1}$ & $\square_{2}$ & $\square_{3}$ & $\square_{4}$ \\
\hline TC034Q06NA & $\begin{array}{l}\text { Expliquer les liens existant entre la biologie, } \\
\text { la physique et la chimie }\end{array}$ & $\square_{1}$ & $\square_{2}$ & $\square_{3}$ & $\square_{4}$ \\
\hline Bouton d'aide & \multicolumn{5}{|c|}{$\begin{array}{l}\text { Votre }<\text { discipline scientifique }>\text { correspond à une discipline scientifique particulière dont votre }<\text { cours de sciences }> \\
\text { principal fait partie. Si vous enseignez le même nombre d'heures pour plusieurs }<\text { cours de sciences }>\text { différents, } \\
\text { choisissez-en un seul pour vos réponses. }\end{array}$} \\
\hline
\end{tabular}

\section{PARTIE B QUESTIONNAIRE À L'INTENTION DE L'ENSEIGNANT(E)}

\section{Renseignements généraux}

\section{TC001 \\ Êtes-vous une femme ou un homme?}

(Sélectionnez une réponse.)

Une femme

Un homme

TCOO2

TC002Q01NA
Quel âge avez-vous ?

(Positionnez le curseur sur le nombre d'années correspondant.)

Nombre d'années :

Curseur : position initiale ; plage : « 20 ans ou moins » - 70 ans ou plus » ; graduation=1.

TC004

TC004Q01NA

Sous quel contrat de travail enseignez-vous dans cet établissement?

(Sélectionnez une réponse.)

Contrat de travail permanent (à durée indéterminée sans expiration prévue avant l'âge de la retraite)

Contrat de travail à durée déterminée de plus d'une année scolaire

Contrat de travail à durée déterminée d'une année scolaire ou moins

\section{Actuellement, enseignez-vous à temps plein ou à temps partiel ?}

TC005

(Tenez compte de tous vos postes actuels d'enseignant(e) pour déterminer si vous enseignez à temps plein ou à temps partiel.)

(Sélectionnez une réponse par ligne.)

TC005Q01N

TC005Q02NA

Mon poste dans cet établissement

L'ensemble de mes postes d'enseignant(e)

\begin{tabular}{|c|c}
$\begin{array}{c}\text { À temps plein } \\
\text { (plus de } 90 \% \\
\text { du temps } \\
\text { de travail } \\
\text { à temps plein) }\end{array}$ \\
\hline$\square_{1}$ \\
\hline$\square_{1}$
\end{tabular}

À temps partiel À temps partiel (entre 71 et $\quad$ (entre 50 et $90 \% \quad 70 \%$ du temps de travail du temps de travail à temps plein) à temps plein)

À temps partiel (moins de $50 \%$ du temps de travail à temps plein) 
TC006

TC006Q01NA

Dans combien d'établissements avez-vous travaillé au cours de votre carrière d'enseignant(e) ?

(Prenez en compte tous les établissements, même si vous avez travaillé dans plusieurs d'entre eux simultanément.) (Positionnez le curseur sur le nombre correspondant d'établissements.)

Nombre d'établissements :

Curseur : position initiale ; plage : « 1 établissement » - « 20 établissements ou plus » ; graduation=1

TC007

TC007Q01NA

TC007Q02NA

Combien d'années d'expérience professionnelle avez-vous?

(Arrondissez les nombres d'années à l'unité, que vous ayez travaillé à temps partiel ou à temps plein. Positionnez le curseur sur le nombre d'années correspondant. Indiquez « 0 » [zéro] si une des options ne correspond pas à votre situation.)

Curseur : position initiale ; plage : « 0 année » - « 50 années ou plus » ; graduation=1.

Contrôle de cohérence/rappel si la réponse à la question TC007Q01 NA est supérieure à celle donnée à la question TC007Q02NA.

\section{Votre formation initiale et votre formation continue}

TC012 TC012Q01NA

TC013

TC013Q01NA

TC014

TC014Q01NA

TC015

TC015Q01NA
Quel est le niveau du diplôme le plus élevé que vous ayez obtenu dans l'enseignement institutionnel ?

(Sélectionnez une réponse.)

\begin{tabular}{l|c}
\hline <Inférieur au Niveau 5 de la CITE> & $\square_{1}$ \\
\hline <Niveau 5B de la CITE $>$ & $\square_{2}$ \\
\hline <Niveau 5A de la CITE - Bachelier / licence> & $\square_{3}$ \\
\hline$<$ Niveau 5A de la CITE - Master> & $\square_{4}$ \\
\hline$<$ Niveau 6 de la CITE $>$ & $\square_{5}$
\end{tabular}

À la fin du <Niveau 3 de la CITE ou inférieur>, aviez-vous pour objectif de poursuivre une carrière dans l'enseignement?

(Sélectionnez une réponse.)

\begin{tabular}{l|l} 
Oui & $\square_{1}$ \\
\hline Non & $\square_{2}$ \\
\hline
\end{tabular}

Avez-vous terminé une formation ou des études d'enseignant ?

(Sélectionnez une réponse.)

\begin{tabular}{l|l}
\hline Oui & $\square_{1}$ \\
\hline Non & $\square_{2}$
\end{tabular}

\section{Comment avez-vous obtenu les qualifications requises pour enseigner?}

(Sélectionnez une réponse.)

J'ai suivi une formation ou des études classiques d'enseignant en fréquentant un <institut de formation pédagogique habilité à former des enseignants>.

J'ai suivi une formation ou des études d'enseignant dans le cadre de la formation continue.

J'ai suivi une formation ou des études d'enseignant sur le lieu de travail.

J'ai suivi une formation dans une autre profession à caractère pédagogique.

Autre

\begin{tabular}{c|c}
$\square_{1}$ \\
$\square_{2}$ \\
$\square_{3}$ \\
$\square_{4}$ \\
$\square_{5}$
\end{tabular}


Les groupes de matières suivants figuraient-ils au programme de votre formation d'enseignant ou d'une autre qualification professionnelle ? Enseignez-vous ces matières en <grade modal du pays pour les élèves de $\mathbf{1 5}$ ans> au cours de cette année scolaire ?

(Comme il s'agit d'une enquête internationale, nous avons dû choisir des catégories assez vastes et y classer de nombreuses matières enseignées à l'école. Si l'intitulé exact d'une de vos matières ne figure pas dans la liste, sélectionnez la catégorie qui vous semble le mieux correspondre à cette matière.)

(Si vous avez besoin d'explications au sujet des termes utilisés dans cette question, utilisez le bouton d'aide.) (Sélectionez toutes les réponses qui conviennent.)

\begin{tabular}{|c|c|c|c|}
\hline & & & \\
\hline TC018Q01N & Lecture, expression écrite et littérature & $\square_{1}$ & $\square_{1}$ \\
\hline TC018Q02N & Mathématiques & $\square_{1}$ & $\square_{1}$ \\
\hline TC018Q03N & Sciences & $\square_{1}$ & $\square_{1}$ \\
\hline TC018Q04N & Technologie & $\square_{1}$ & $\square_{1}$ \\
\hline TC018Q05N & Sciences humaines & $\square_{1}$ & $\square_{1}$ \\
\hline TC018Q06N & Langues vivantes étrangères & $\square_{1}$ & $\square_{1}$ \\
\hline TC018Q07N & Langues anciennes (par ex. le latin) & $\square_{1}$ & $\square_{1}$ \\
\hline TC018Q08N & Disciplines artistiques & $\square_{1}$ & $\square_{1}$ \\
\hline TC018Q09N & Éducation physique & $\square_{1}$ & $\square_{1}$ \\
\hline TC018Q10N & Religion et/ou morale & $\square_{1}$ & $\square_{1}$ \\
\hline TC018Q11N & Disciplines pratiques et professionnelles & $\square_{1}$ & $\square_{1}$ \\
\hline
\end{tabular}

Bouton d'aide Lecture, expression écrite et littérature : la lecture et l'expression écrite (et la littérature) dans la langue maternelle, dans la langue d'enseignement et dans la langue nationale (ou régionale) en tant que deuxième langue (pour les allophones), la linguistique, l'expression orale et la littérature

Mathématiques : les mathématiques, les statistiques, la géométrie, l'algèbre, etc.

Sciences : les sciences naturelles, la physique, la chimie, la biologie, la biologie humaine, les sciences de la Terre et de l'Univers, les sciences de l'environnement, ainsi que l'agronomie, I'horticulture et la foresterie Technologie : I'initiation à la technologie, dont les technologies de l'information, l'informatique, la construction et la topométrie, l'électronique, le graphisme et le design, la dactylographie, le traitement de texte, les techniques d'atelier et le dessin technique

Sciences humaines : les sciences humaines, l'étude des collectivités, l'étude des sociétés contemporaines,

l'économie, l'étude du milieu, la géographie, l'histoire, les sciences sociales, le droit, l'étude du pays, la pensée éthique et la philosophie

Langues vivantes étrangères : les langues vivantes autres que la langue d'enseignement

Langues anciennes (par ex. le latin)

Disciplines artistiques : les arts, la musique, les arts visuels, les arts appliqués, le théâtre, les arts du spectacle, la photographie, le dessin, l'artisanat et la couture artistique

Éducation physique : I'éducation physique, la gymnastique, la danse et l'hygiène

Religion et/ou morale : la religion, I'histoire des religions, la culture religieuse et la morale

Disciplines pratiques et professionnelles : les enseignements à vocation professionnelle (qui préparent à exercer un métier spécifique), la technique, les arts ménagers, la comptabilité, le commerce, l'enseignement professionnel, l'habillement et la couture, les cours de conduite, l'économie ménagère, les cours polytechniques, le secrétariat, le tourisme et l'accueil, et l'artisanat. 
TC020

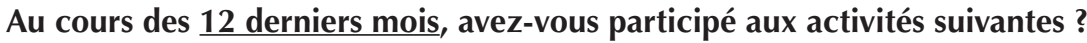

(Sélectionnez une réponse par ligne.)

\begin{tabular}{l|l|c|c}
\multicolumn{1}{l|}{} & Oui & Non \\
\hline TC020Q01NA & À un programme de qualification (par ex. <sanctionné par un diplôme>) & $\square_{1}$ & $\square_{2}$ \\
\hline TC020Q02NA & $\begin{array}{l}\text { Aux activités d'un réseau d'enseignants axé sur la formation continue } \\
\text { des enseignants }\end{array}$ & $\square_{1}$ & $\square_{2}$ \\
\hline TC020Q03NA & $\begin{array}{l}\text { À des recherches individuelles ou en groupe sur un sujet en rapport } \\
\text { avec votre profession }\end{array}$ & $\square_{1}$ & $\square_{2}$ \\
\hline TC020Q04NA & $\begin{array}{l}\text { À des activités de tutorat, d'observation collégiale ou de coaching officiellement } \\
\text { organisées dans votre établissement }\end{array}$ & $\square_{1}$ & $\square_{2}$ \\
\hline TC020Q05NA & $\begin{array}{l}\text { Lecture d'ouvrages spécialisés (par ex. des revues, des travaux } \\
\text { de recherche, des thèses). }\end{array}$ & $\square_{1}$ & $\square_{2}$ \\
\hline TC020Q06NA & Dialogue informel avec vos collègues sur l'amélioration de votre enseignement & $\square_{1}$ & $\square_{2}$ \\
\hline
\end{tabular}

TC021

TC021Q01NA

TC045
Êtes-vous tenu(e) de participer à des activités de formation continue ?

(Sélectionnez une réponse.)

\begin{tabular}{l} 
Oui \\
\hline Non
\end{tabular}
Les éléments suivants figuraient-ils au programme de votre formation d'enseignant (ou d'une autre qualification professionnelle) et/ou dans vos activités de formation continue ? (Sélectionnez toutes les réponses qui conviennent.)

\begin{tabular}{|c|c|c|c|}
\hline & & $\begin{array}{c}\text { Figurait } \\
\text { au programme } \\
\text { de ma formation } \\
\text { d'enseignant } \\
\text { ou d'une autre } \\
\text { qualification } \\
\text { professionnelle } \\
\end{array}$ & $\begin{array}{c}\text { Figurait } \\
\text { dans mes activités } \\
\text { de formation } \\
\text { continue au cours } \\
\text { des } 12 \text { derniers mois }\end{array}$ \\
\hline TC045Q01N & Connaissance et maitrise de la ou des matière(s) que j'enseigne & $\square_{1}$ & $\square_{1}$ \\
\hline TC045Q02N & Compétences pédagogiques dans la ou les matière(s) que j'enseigne & $\square_{1}$ & $\square_{1}$ \\
\hline TC045Q03N & Connaissance des programmes de cours & $\square_{1}$ & $\square_{1}$ \\
\hline TC045Q04N & Pratiques d'évaluation des élèves & $\square_{1}$ & $\square_{1}$ \\
\hline TC045Q05N & $\begin{array}{l}\text { Compétences en TIC (technologies de l'information et de la communication) } \\
\text { à l'appui de l'enseignement }\end{array}$ & $\square_{1}$ & $\square_{1}$ \\
\hline TC045Q06N & Gestion de la classe et du comportement des élèves & $\square_{1}$ & $\square_{1}$ \\
\hline TC045Q07N & Gestion et administration de l'établissement & $\square_{1}$ & $\square_{1}$ \\
\hline TC045Q08N & Approches pédagogiques individualisées & $\square_{1}$ & $\square_{1}$ \\
\hline TC045Q09N & Prise en charge d'élèves présentant des besoins éducatifs particuliers & $\square_{1}$ & $\square_{1}$ \\
\hline TC045Q10N & Enseignement en milieu multiculturel ou plurilingue & $\square_{1}$ & $\square_{1}$ \\
\hline TC045Q11N & $\begin{array}{l}\text { Enseignement de compétences transversales (résolution de problèmes, } \\
\text { méthodes d'apprentissage) }\end{array}$ & $\square_{1}$ & $\square_{1}$ \\
\hline TC045Q12N & Conseil et orientation professionnelle des élèves & $\square_{1}$ & $\square_{1}$ \\
\hline TC045Q13N & Évaluation interne ou autoévaluation des établissements & $\square_{1}$ & $\square_{1}$ \\
\hline TC045Q14N & Exploitation des résultats d'évaluation & $\square_{1}$ & $\square_{1}$ \\
\hline TC045Q15N & Coopération entre parents et enseignants & $\square_{1}$ & $\square_{1}$ \\
\hline
\end{tabular}




\section{Votre établissement}

TC028

L'enseignement que votre établissement est à même de dispenser est-il affecté par les problèmes suivants?

(Sélectionnez une réponse par ligne.)

\begin{tabular}{|c|c|c|c|c|c|}
\hline & & Pas du tout & Très peu & $\begin{array}{l}\text { Dans } \\
\text { une certaine } \\
\text { mesure }\end{array}$ & Beaucoup \\
\hline TC028Q01NA & Manque de personnel enseignant & $\square_{1}$ & $\square_{2}$ & $\square_{3}$ & $\square_{4}$ \\
\hline TC028Q02NA & Personnel enseignant inadéquat ou peu qualifié & $\square_{1}$ & $\square_{2}$ & $\square_{3}$ & $\square_{4}$ \\
\hline TC028Q03NA & Manque de personnel auxiliaire & $\square_{1}$ & $\square_{2}$ & $\square_{3}$ & \\
\hline TC028Q04NA & Personnel auxiliaire inadéquat ou peu qualifié & $\square_{1}$ & $\square_{2}$ & $\square_{3}$ & $\square_{4}$ \\
\hline TC028Q05NA & $\begin{array}{l}\text { Manque de matériel pédagogique (par ex. manuels } \\
\text { scolaires, équipement informatique, matériel } \\
\text { de bibliothèque ou de laboratoire) }\end{array}$ & $\square_{1}$ & $\square_{2}$ & $\square_{3}$ & 4 \\
\hline TC028Q06NA & $\begin{array}{l}\text { Matériel pédagogique inadéquat ou de mauvaise qualité } \\
\text { (par ex. manuels scolaires, équipement informatique, } \\
\text { matériel de bibliothèque ou de laboratoire) }\end{array}$ & $\square_{1}$ & 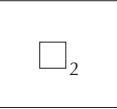 & $\square_{3}$ & 4 \\
\hline TC028Q07NA & $\begin{array}{l}\text { Manque d'infrastructures (par ex. bâtiments, terrain, } \\
\text { chauffage/climatisation, systèmes d'éclairage } \\
\text { et acoustiques) }\end{array}$ & $\square_{1}$ & $\square_{2}$ & $\square_{3}$ & ]$_{4}$ \\
\hline TC028Q08NA & $\begin{array}{l}\text { Infrastructures inadéquates ou de mauvaise qualité } \\
\text { (par ex. bâtiments, terrain, chauffage/ climatisation, } \\
\text { systèmes d'éclairage et acoustiques) }\end{array}$ & $\square_{1}$ & $\square_{2}$ & $\square_{3}$ & $\square_{4}$ \\
\hline
\end{tabular}

Dans quelle mesure êtes-vous d'accord ou non avec les affirmations suivantes à propos de votre établissement?

(Sélectionnez une réponse par ligne.)

\begin{tabular}{c|l|c|c|c|c}
\multicolumn{2}{c|}{} & $\begin{array}{c}\text { Pas du tout } \\
\text { d'accord }\end{array}$ & $\begin{array}{c}\text { Pas } \\
\text { d'accord }\end{array}$ & $\begin{array}{c}\text { Tout à fait } \\
\text { D'accord } \\
\text { d'accord }\end{array}$ \\
\hline TC060Q02NA & $\begin{array}{l}\text { Le chef d'établissement vise un consensus avec l'ensemble } \\
\text { du personnel quand il détermine les priorités et objectifs } \\
\text { de l'établissement. }\end{array}$ & $\square_{1}$ & $\square_{2}$ & $\square_{3}$ & $\square_{4}$ \\
\hline TC060Q04NA & Le chef d'établissement est conscient de mes besoins. & $\square_{1}$ & $\square_{2}$ & $\square_{3}$ & $\square_{4}$ \\
\hline TC060Q06NA & $\begin{array}{l}\text { Le chef d'établissement suscite de nouvelles idées } \\
\text { pour mon apprentissage professionnel. }\end{array}$ & $\square_{1}$ & $\square_{2}$ & $\square_{3}$ & $\square_{4}$ \\
\hline TC060Q07NA & $\begin{array}{l}\text { Le chef d'établissement traite le personnel enseignant } \\
\text { en professionnels. }\end{array}$ & $\square_{1}$ & $\square_{2}$ & $\square_{3}$ & $\square_{4}$ \\
\hline TC060Q09NA & $\begin{array}{l}\text { Le chef d'établissement veille à nous impliquer } \\
\text { dans la prise de décisions. }\end{array}$ & $\square_{1}$ & $\square_{2}$ & $\square_{3}$ & $\square_{4}$ \\
\hline
\end{tabular}


TC046

À quelle fréquence vous livrez-vous aux activités suivantes dans cet établissement, en moyenne ? (Sélectionnez une réponse par ligne.)

\begin{tabular}{|c|c|c|c|c|c|c|c|}
\hline & & Jamais & $\begin{array}{l}\text { Pas plus } \\
\text { de } 1 \text { fois } \\
\text { par an }\end{array}$ & $\begin{array}{l}\text { Entre } 2 \\
\text { et } 4 \text { fois } \\
\text { par an }\end{array}$ & $\begin{array}{l}\text { Entre } 5 \\
\text { et } 10 \text { fois } \\
\text { par an }\end{array}$ & $\begin{array}{l}\text { Entre } 1 \\
\text { et } 3 \text { fois } \\
\text { par mois }\end{array}$ & $\begin{array}{l}\text { Au moins } \\
1 \text { fois par } \\
\text { semaine }\end{array}$ \\
\hline TC046Q01NA & $\begin{array}{l}\text { Faire cours en équipe dans la même } \\
\text { classe }\end{array}$ & & & & & $\square_{5}$ & 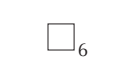 \\
\hline TC046Q02NA & $\begin{array}{l}\text { Observer le travail d'autres enseignants } \\
\text { en classe et le commenter }\end{array}$ & & & & & & \\
\hline TC046Q03NA & $\begin{array}{l}\text { Participer à des activités organisées } \\
\text { collectivement pour plusieurs classes et } \\
\text { groupes d'âge (par ex. des projets, etc.) }\end{array}$ & & & & & & \\
\hline TC046Q04NA & $\begin{array}{l}\text { Échanger du matériel pédagogique } \\
\text { avec mes collègues }\end{array}$ & & & & & & \\
\hline TC046Q05NA & $\begin{array}{l}\text { Discuter des progrès faits par certains } \\
\text { élèves }\end{array}$ & $\square_{1}$ & $\square_{2}$ & $\square_{3}$ & $\square$ & & 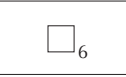 \\
\hline TC046Q06NA & $\begin{array}{l}\text { Collaborer avec d'autres enseignants } \\
\text { de mon établissement pour appliquer } \\
\text { des barèmes communs pour évaluer } \\
\text { les progrès des élèves }\end{array}$ & $\square_{1}$ & $\bigsqcup_{2}$ & $\square_{3}$ & & & \\
\hline TC046Q07NA & Assister à des réunions d'équipes & $\square_{1}$ & $\square_{2}$ & $\square_{3}$ & $\square_{4}$ & $\square_{5}$ & $\square_{6}$ \\
\hline TC046Q08NA & $\begin{array}{l}\text { Participer à des activités de formation } \\
\text { professionnelle en groupe }\end{array}$ & $\square_{1}$ & $\square_{2}$ & $\square_{3}$ & $\square_{4}$ & $\square_{5}$ & $\square_{6}$ \\
\hline
\end{tabular}

Nous aimerions savoir quels sentiments vos fonctions vous inspirent dans l'ensemble. Dans quelle mesure êtes-vous d'accord avec les affirmations suivantes?

(Sélectionnez une réponse par ligne.)

\begin{tabular}{|c|c|c|c|c|c|}
\hline & & $\begin{array}{l}\text { Pas du tout } \\
\text { d'accord }\end{array}$ & $\begin{array}{c}\text { Pas } \\
\text { d'accord }\end{array}$ & D'accord & $\begin{array}{l}\text { Tout à fait } \\
\text { d'accord }^{\prime}\end{array}$ \\
\hline TC026Q01NA & $\begin{array}{l}\text { Les avantages du métier d'enseignant compensent } \\
\text { largement ses inconvénients. }\end{array}$ & & & & \\
\hline TC026Q02NA & $\begin{array}{l}\text { Si c'était à refaire, je choisirais de nouveau le métier } \\
\text { d'enseignant(e). }\end{array}$ & & & & \\
\hline TC026Q04NA & Je regrette ma décision de devenir enseignant(e). & & & & \\
\hline TC026Q05NA & J'aime travailler dans cet établissement. & & & & \\
\hline TC026Q06NA & $\begin{array}{l}\text { Je me demande si je n'aurais pas mieux fait de choisir } \\
\text { une autre profession. }\end{array}$ & & & & \\
\hline TC026Q07NA & $\begin{array}{l}\text { Mon établissement est un endroit agréable où travailler, } \\
\text { je le recommanderais à d'autres enseignants. }\end{array}$ & $\square_{1}$ & & & \\
\hline TC026Q09NA & Je suis satisfait(e) de mon travail dans cet établissement. & $\square_{1}$ & $\square_{2}$ & $\square_{3}$ & $\square_{4}$ \\
\hline TC026Q10NA & Dans l'ensemble, mon travail me donne satisfaction. & $\square_{1}$ & $\square_{2}$ & $\square_{3}$ & $\square_{4}$ \\
\hline
\end{tabular}




\section{Les pratiques pédagogiques}

\begin{tabular}{|c|c|c|c|c|c|c|c|}
\hline \multirow[t]{2}{*}{ TC048 } & \multicolumn{7}{|c|}{$\begin{array}{l}\text { À quelle fréquence organisez-vous les activités suivantes avec vos élèves? } \\
\text { (Sélectionnez une réponse par ligne.) }\end{array}$} \\
\hline & & $\begin{array}{l}\text { Jamais } \\
\text { ou } \\
\text { presque } \\
\text { jamais }\end{array}$ & $\begin{array}{l}\text { Pas plus } \\
\text { de } 1 \text { fois } \\
\text { par an }\end{array}$ & $\begin{array}{l}\text { Entre } 2 \\
\text { et } 4 \text { fois } \\
\text { par an }\end{array}$ & $\begin{array}{l}\text { Entre } 5 \\
\text { et } 9 \text { fois } \\
\text { par an }\end{array}$ & $\begin{array}{l}\text { Entre } 1 \\
\text { et } 3 \text { fois } \\
\text { par mois }\end{array}$ & $\begin{array}{l}\text { Au moins } \\
1 \text { fois par } \\
\text { semaine }\end{array}$ \\
\hline TC048Q01NA & $\begin{array}{l}\text { Activités courtes } \\
\text { (entre } 10 \text { minutes et } 2 \text { heures) en équipe, } \\
\text { par ex. des exercices ou des problèmes }\end{array}$ & $\square_{1}$ & $\square_{2}$ & $\square_{3}$ & $\square_{4}$ & $\square_{5}$ & $\square_{6}$ \\
\hline TC048Q02NA & $\begin{array}{l}\text { Projets plus longs (sur plusieurs semaines) } \\
\text { en équipe, par ex. rédiger un document, } \\
\text { concevoir quelque chose, etc. }\end{array}$ & $\square_{1}$ & $\square_{2}$ & $\square_{3}$ & $\square$ & $\square_{5}$ & $\square_{6}$ \\
\hline TC048Q03NA & $\begin{array}{l}\text { Préparer et présenter } \\
\text { un exposé/une présentation en équipe }\end{array}$ & $\square_{1}$ & $\square_{2}$ & $\square_{3}$ & $\square_{4}$ & $\square_{5}$ & $\square_{6}$ \\
\hline
\end{tabular}

TC051

À quelle fréquence donnez-vous les types d'appréciation suivants lors des activités en équipe de vos élèves?

(Sélectionnez une réponse par ligne.)

\begin{tabular}{l|l|c|c|c|c}
\multicolumn{2}{l|}{} & $\begin{array}{c}\text { Jamais ou } \\
\text { presque } \\
\text { jamais }\end{array}$ & Parfois & $\begin{array}{c}\text { Toujours } \\
\text { ou presque } \\
\text { toujours }\end{array}$ \\
\hline TC051Q01NA & Je ne donne pas d'appréciations & $\square_{1}$ & $\square_{2}$ & $\square_{3}$ & $\square_{4}$ \\
\hline TC051Q02NA & $\begin{array}{l}\text { Des appréciations individuelles pour des performances } \\
\text { individuelles }\end{array}$ & $\square_{1}$ & $\square_{2}$ & $\square_{3}$ & $\square_{4}$ \\
\hline TC051Q03NA & Des appréciations collectives pour un résultat d'équipe & $\square_{1}$ & $\square_{2}$ & $\square_{3}$ & $\square$ \\
\hline TC051Q04NA & Des appréciations collectives sur des apports individuels & $\square_{1}$ & $\square_{2}$ & $\square_{3}$ & $\square_{4}$ \\
\hline TC051Q05NA & Des appréciations individuelles pour un résultat d'équipe & $\square_{1}$ & $\square_{2}$ & $\square_{3}$ & $\square_{4}$ \\
\hline
\end{tabular}

$\mathrm{TC052}$

À quelle fréquence utilisez-vous les types de collaboration suivants lors des activités en équipe de vos élèves?

(Sélectionnez une réponse par ligne.)

\begin{tabular}{c|l|c|c|c|c}
\multicolumn{2}{l|}{} & $\begin{array}{c}\text { Jamais ou } \\
\text { presque } \\
\text { jamais }\end{array}$ & Parfois & $\begin{array}{c}\text { Toujours } \\
\text { Souvent } \\
\text { ou presque } \\
\text { toujours }\end{array}$ \\
\hline TC052Q01NA & $\begin{array}{l}\text { Les membres d'une équipe répartissent le travail } \\
\text { selon la spécialisation de chacun d'eux }\end{array}$ & $\square_{1}$ & $\square_{2}$ & $\square_{3}$ & $\square_{4}$ \\
\hline TC052Q02NA & Les membres d'une équipe travaillent à un résultat collectif & $\square_{1}$ & $\square_{2}$ & $\square_{3}$ & $\square_{4}$ \\
\hline TC052Q03NA & $\begin{array}{l}\text { Les membres d'une équipe reçoivent des informations } \\
\text { différentes (interdépendance des ressources) }\end{array}$ & $\square_{1}$ & $\square_{2}$ & $\square_{3}$ & $\square_{4}$ \\
\hline TC052Q04NA & $\begin{array}{l}\text { Les membres d'une équipe se voient attribuer des rôles } \\
\text { différents (interdépendance des rôles) }\end{array}$ & $\square_{1}$ & $\square_{2}$ & $\square_{3}$ & $\square_{4}$ \\
\hline
\end{tabular}

TC053

À quelle fréquence utilisez-vous les méthodes de regroupement suivantes lors des activités en équipe de vos élèves ?

(Sélectionnez une réponse par ligne.)

\begin{tabular}{l|l|c|c|c|c}
\multicolumn{2}{l|}{} & $\begin{array}{c}\text { Jamais ou } \\
\text { presque } \\
\text { jamais }\end{array}$ & Parfois & $\begin{array}{c}\text { Toujours } \\
\text { ou presque } \\
\text { Soujours }\end{array}$ \\
\hline TC053Q01NA & Des groupes d'élèves présentant des aptitudes variées & $\square_{1}$ & $\square_{2}$ & $\square_{3}$ & $\square_{4}$ \\
\hline TC053Q02NA & Des groupes d'élèves présentant des aptitudes similaires & $\square_{1}$ & $\square_{2}$ & $\square_{3}$ & $\square_{4}$ \\
\hline TC053Q03NA & Des groupes formés librement par les élèves & $\square_{1}$ & $\square_{2}$ & $\square_{3}$ & $\square_{4}$ \\
\hline
\end{tabular}




\begin{tabular}{|c|c|c|c|c|c|}
\hline & & $\begin{array}{l}\text { Jamais ou } \\
\text { presque } \\
\text { jamais }\end{array}$ & Parfois & Souvent & $\begin{array}{l}\text { À chaque } \\
\text { cours ou } \\
\text { presque }\end{array}$ \\
\hline TC054Q01NA & J'administre un test que j'élabore moi-même. & $\square_{1}$ & $\square_{2}$ & $\square_{3}$ & $\square_{4}$ \\
\hline TC054Q02NA & J'administre un <test standardisé>. & $\square_{1}$ & $\square_{2}$ & $\square_{3}$ & $\square_{4}$ \\
\hline TC054Q03NA & $\begin{array}{l}\text { Je demande à un élève de répondre à des questions devant } \\
\text { la classe. }\end{array}$ & $\square_{1}$ & $\square_{2}$ & $\square_{3}$ & $\square_{4}$ \\
\hline TC054Q04NA & $\begin{array}{l}\text { J'ajoute un commentaire écrit à <la note chiffrée } \\
\text { ou la lettre> attribuée au travail de l'élève. }\end{array}$ & $\square_{1}$ & $\square_{2}$ & $\square_{3}$ & $\square_{4}$ \\
\hline TC054Q05NA & Je laisse les élèves s'évaluer eux-mêmes. & $\square_{1}$ & $\square_{2}$ & $\square_{3}$ & $\square_{4}$ \\
\hline TC054Q06NA & $\begin{array}{l}\text { J'observe les élèves pendant qu'ils effectuent une tâche } \\
\text { particulière en classe et je leur fais des commentaires } \\
\text { sur leur travail. }\end{array}$ & $\square_{1}$ & $\square_{2}$ & $\square_{3}$ & $\square_{4}$ \\
\hline TC054Q07NA & $\begin{array}{l}\text { Je rassemble des données provenant d'exercices faits } \\
\text { en classe ou de devoirs faits à la maison. }\end{array}$ & $\square_{1}$ & $\square_{2}$ & $\square_{3}$ & $\square_{4}$ \\
\hline Bouton d'aide & \multicolumn{5}{|c|}{$\begin{array}{l}\text { Dans ce contexte, le terme <tests standardisé> fait référence aux tests standardisés obligatoires (imposés } \\
\text { par exemple par les autorités nationales, régionales ou locales) et aux tests standardisés non obligatoires } \\
\text { (par ex. des tests standardisés accessibles au public ou disponibles dans le commerce). Ces tests présentent } \\
\text { une cohérence dans leur conception, leur contenu, leur administration et leur système de notation. } \\
\text { Les résultats peuvent être comparés entre élèves et entre établissements. }\end{array}$} \\
\hline
\end{tabular}

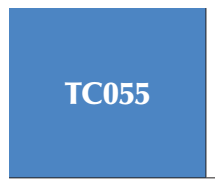

Dans quelle mesure utilisez-vous les approches suivantes pour attribuer les notes de fin de <semestre> aux élèves du < grade modal du pays pour les élèves de $\mathbf{1 5}$ ans> ?

(Si vous avez besoin d'explications au sujet du terme "<test standardisé> ", utilisez le bouton d'aide.)

(Sélectionnez une réponse par ligne.)

\begin{tabular}{|c|c|c|c|c|c|}
\hline & & Pas du tout & Très peu & $\begin{array}{l}\text { Dans une } \\
\text { certaine } \\
\text { mesure }\end{array}$ & $\begin{array}{l}\text { Dans une } \\
\text { grande } \\
\text { mesure }\end{array}$ \\
\hline TC055Q01NA & $\begin{array}{l}\text { Je tiens compte des progrès réalisés par chaque élève } \\
\text { depuis le début du < semestre }>\text {. }\end{array}$ & $\square_{1}$ & $\square_{2}$ & $\square_{3}$ & $\square_{4}$ \\
\hline TC055Q02NA & $\begin{array}{l}\text { Je tiens compte de l'aptitude de l'élève à résoudre } \\
\text { un problème. }\end{array}$ & $\square_{1}$ & $\square_{2}$ & $\square_{3}$ & $\square_{4}$ \\
\hline TC055Q03NA & Je tiens compte de l'esprit critique de l'élève. & $\square_{1}$ & $\square_{2}$ & $\square_{3}$ & $\square_{4}$ \\
\hline TC055Q04NA & $\begin{array}{l}\text { Je tiens compte de la performance des élèves dans } \\
\text { les activités en équipe de résolution de problèmes. }\end{array}$ & $\square_{1}$ & $\square_{2}$ & $\square_{3}$ & $\square_{4}$ \\
\hline TC055Q05NA & $\begin{array}{l}\text { Je tiens compte des efforts fournis par l'élève, même si } \\
\text { ses résultats n'ont pas progressé. }\end{array}$ & $\square_{1}$ & $\square_{2}$ & $\square_{3}$ & $\square_{4}$ \\
\hline TC055Q06NA & $\begin{array}{l}\text { Je compare les performances des élèves du cours actuel } \\
\text { avec celles des élèves du cours de l'an passé. }\end{array}$ & $\square_{1}$ & $\square_{2}$ & $\square_{3}$ & $\square_{4}$ \\
\hline TC055Q07NA & $\begin{array}{l}\text { Je compare les résultats d'un élève à ceux des autres } \\
\text { élèves du cours. }\end{array}$ & $\square_{1}$ & $\square_{2}$ & $\square_{3}$ & $\square_{4}$ \\
\hline TC055Q08NA & $\begin{array}{l}\text { J'évalue les résultats des élèves par rapport à des }<\text { normes } \\
\text { de résultats publiées au niveau national ou régional }>\text {. }\end{array}$ & $\square_{1}$ & $\square_{2}$ & $\square_{3}$ & $\square_{4}$ \\
\hline TC055Q11NA & $\begin{array}{l}\text { Je tiens compte du niveau de participation au cours } \\
\text { de l'élève. }\end{array}$ & $\square_{1}$ & $\square_{2}$ & $\square_{3}$ & $\square 4$ \\
\hline TC055Q13NA & $\begin{array}{l}\text { J'appuie ma notation sur les <tests standardisés }>\text { imposés } \\
\text { par les autorités nationales, régionales ou locales, comme } \\
<\text { exemple spécifique au pays }>\text {. }\end{array}$ & $\square_{1}$ & $\square_{2}$ & $\square_{3}$ & $\square_{4}$ \\
\hline TC055Q14NA & $\begin{array}{l}\text { J'appuie ma notation sur les }<\text { tests standardisés }> \\
\text { non obligatoires accessibles au public ou disponibles } \\
\text { dans le commerce, comme }<\text { exemple spécifique } \\
\text { au pays }>\text {. }\end{array}$ & $\square_{1}$ & $\square_{2}$ & $\square_{3}$ & $\square$ प \\
\hline Bouton d'aide & \multicolumn{5}{|c|}{$\begin{array}{l}\text { Les <tests standardisés> présentent une cohérence dans leur conception, leur contenu, leur administration } \\
\text { et leur système de notation. Les résultats peuvent être comparés entre élèves et entre établissements. } \\
\text { Les tests élaborés par les enseignants sont exclus! }\end{array}$} \\
\hline
\end{tabular}





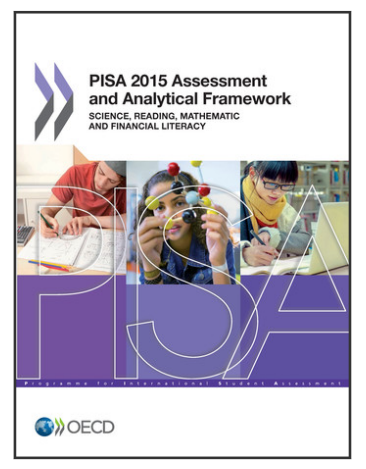

Extrait de :

PISA 2015 Assessment and Analytical Framework

Science, Reading, Mathematic and Financial Literacy

Accéder à cette publication :

https://doi.org/10.1787/9789264255425-en

\section{Merci de citer ce chapitre comme suit :}

OCDE (2016), « Questionnaires contextuels de l'enquete PISA 2015 », dans PISA 2015 Assessment and Analytical Framework : Science, Reading, Mathematic and Financial Literacy, Éditions OCDE, Paris.

DOI: https://doi.org/10.1787/9789264259478-8-fr

Cet ouvrage est publié sous la responsabilité du Secrétaire général de l'OCDE. Les opinions et les arguments exprimés ici ne reflètent pas nécessairement les vues officielles des pays membres de l'OCDE.

Ce document et toute carte qu'il peut comprendre sont sans préjudice du statut de tout territoire, de la souveraineté s'exerçant sur ce dernier, du tracé des frontières et limites internationales, et du nom de tout territoire, ville ou région.

Vous êtes autorisés à copier, télécharger ou imprimer du contenu OCDE pour votre utilisation personnelle. Vous pouvez inclure des extraits des publications, des bases de données et produits multimédia de l'OCDE dans vos documents, présentations, blogs, sites Internet et matériel d'enseignement, sous réserve de faire mention de la source OCDE et du copyright. Les demandes pour usage public ou commercial ou de traduction devront être adressées à rights@oecd.org. Les demandes d'autorisation de photocopier une partie de ce contenu à des fins publiques ou commerciales peuvent être obtenues auprès du Copyright Clearance Center (CCC) info@copyright.com ou du Centre français d'exploitation du droit de copie (CFC) contact@cfcopies.com. 